\title{
SUBFORMULA AND SEPARATION PROPERTIES IN NATURAL DEDUCTION VIA SMALL KRIPKE MODELS
}

\author{
PETER MILNE \\ Department of Philosophy, University of Stirling
}

\begin{abstract}
Various natural deduction formulations of classical, minimal, intuitionist, and intermediate propositional and first-order logics are presented and investigated with respect to satisfaction of the separation and subformula properties. The technique employed is, for the most part, semantic, based on general versions of the Lindenbaum and Lindenbaum-Henkin constructions. Careful attention is paid (i) to which properties of theories result in the presence of which rules of inference, and (ii) to restrictions on the sets of formulas to which the rules may be employed, restrictions determined by the formulas occurring as premises and conclusion of the invalid inference for which a counterexample is to be constructed. We obtain an elegant formulation of classical propositional logic with the subformula property and a singularly inelegant formulation of classical first-order logic with the subformula property, the latter, unfortunately, not a product of the strategy otherwise used throughout the article. Along the way, we arrive at an optimal strengthening of the subformula results for classical first-order logic obtained as consequences of normalization theorems by Dag Prawitz and Gunnar Stålmarck.
\end{abstract}

\$1. Introduction. Although what follows can be seen as containing a contribution to the debate over proof-theoretic semantics and the revisionist challenge posed by such authors as Dag Prawitz, Michael Dummett, and Neil Tennant, its original motivation lies in two technical concerns. One of these starts out from the observation that in providing counterexamples to invalid inference patterns one does not follow the sequence set out in standard completeness proofs employing the Lindenbaum and Henkin-Lindenbaum constructions: when we come to construct Kripke models with a view to showing a certain sequent underivable in intuitionist logic, be it propositional or first order, we attend at most to the behavior of subformulas of the formulas in the sequent and, in the first-order case, instances of quantified formulas, subformulas of these, instances of these, and so on, at nodes in a suitable model; we do not attend to all formulas of the language in question, tacitly we let our model take care of the others-soundness guarantees that nothing untoward happens.

Put another way, we can see this as a delimiting of the language of interest, a very narrow delimiting indeed to the formulas of the sequent in question and their subformulas. This ties the first motive to the second: the fact that, given completeness of, say, intuitionist propositional logic with respect to a semantics, the separation property yields completeness results for various subsets of the rules when applied to semantically valid sequents composed of formulas containing only connectives governed by those rules, and the subformula property yields completeness under an even greater restriction, namely that the rules governing connectives that occur in a valid sequent be confined in application

Received: September 15, 2007 
to subformulas of the formulas occurring in that sequent. We have, in other words, completeness for fragments of the original logic, and we have, then, a reason to come at this from the opposite direction, to derive the subformula and separation properties semantically by obtaining appropriate completeness results for fragments of the original logic directly using Kripke semantics, a technique pioneered by Krister Segerberg in obtaining a semantic proof of the separation property for intuitionist propositional logic. ${ }^{1}$ We go further, both in looking at classical propositional logic and first-order logics and in obtaining not only the separation property but, in some cases, the subformula property too. The fundamental thought that underpins the construction of appropriate Kripke models is that we restrict the Lindenbaum and Lindenbaum-Henkin constructions to particular sets of formulas determined by the sequent of interest. ${ }^{2}$

A formulation of a logic, whether sequent calculus or natural deduction, enjoys the (strict) subformula property if any derivable sequent has a derivation employing only subformulas of the premises and conclusion(s). A sequent calculus formulation of a logic enjoys the separation property if any sequent derivable in the logic has a derivation employing neither left nor right introduction rules governing connectives and (where appropriate) quantifiers not occurring in members of the sequent. For natural deduction formulations, which are the focus of attention here, we say: a formulation of a logic enjoys the separation property if any derivable sequent has a derivation employing neither introduction nor elimination rules governing connectives and, where appropriate, quantifiers not occurring in members of the sequent. ${ }^{3}$

In the case of propositional logics, a subformula is any part (not necessarily a proper part) of a formula that is itself a formula. (Here I say 'formula' where others might say 'well-formed formula'.) For first-order logics the notion of a subformula is looser: we must allow among subformulas instances of universal and existential generalizations employing both constants occurring in the sequent in question and "parametric" constants not occurring in the sequent, subformulas of such instances, and, where appropriate, instantiations

1 See the final section, sec. 7, of Segerberg (1968). An algebraic proof of the separation property for intuitionist propositional logic was given by Horn (1962). Horn's proof proceeds by embedding in Heyting algebras algebraic structures of various kinds depending on the connectives occurring in formulas. Horn and Segerberg both work with a Hilbert-style axiomatic formulation of intuitionist propositional logic; in that setting separation means that any theorem of the system is provable using only axioms for the conditional together with those axioms containing connectives that occur in the theorem. One could find a close parallel between Horn's technique and Segerberg's.

2 The Lindenbaum construction is used by Segerberg (1968) for a number of propositional logics, the Lindenbaum-Henkin construction by Fitting (1969, chap. 5, sec. 6) and by Thomason (1970) for first-order intuitionist logic. In proving the weak completeness of intuitionist first-order logic, Kripke (1965) took a rather different approach. (I should perhaps acknowledge that some authors, witness Thomason (1970), do not literally consider all formulas of the language. Rather, they consider only all existential generalizations and all disjunctions, relying on the logical equivalence of $A$ and $A \vee A$ to take care of formulas of other forms. This is hardly an example to follow when interested in the separation and subformula properties.)

3 To be strictly accurate, we should allow for impure rules, introduction and elimination rules in whose formulation connectives and/or quantifiers other than the connectives introduced or eliminated feature. The separation property should then allow that these other connectives may occur in inferences even if they occur in neither premise(s) nor conclusion. Authors differ in their attitudes to impure rules: compare Dummett (1991, pp. 257-258) and Tennant (1997, pp. 314-315). Impure rules play no role in what is to follow (unless one counts the formulations of $\vee$-elimination, Rule of Dilemma, and Tarski's Rule in 3.7 as impure). 
in turn of these, and so on. ${ }^{4}$ When the subformula property fails to hold of a formulation of a logic we may find, nonetheless, that quite tight constraints on the range of formulas occurring in a derivation, determined by the derivable sequent in question and the rules available in the formulation of the logic, are respected-for example, it may be that only subformulas of formulas in the sequent and negations of such need occur in a derivation. It may be that while the subformula property fails to hold, the formulation under consideration enjoys the separation property.

As commonly presented, intuitionist and minimal propositional and first-order logics enjoy the subformula and, therefore, the separation properties. The standard way to demonstrate these facts is to use the sequent calculus formulation of the logic in question and obtain them as corollaries to cut elimination: in a cut-free sequent calculus derivation every formula occurring in the derivation is a subformula of a formula occurring in the antecedent or succeedent of the derived sequent. ${ }^{5}$ Dag Prawitz obtained subformula and separation properties for natural deduction derivations in intuitionist and minimal first-order logic and a restricted subformula property for natural deduction derivations in classical first-order logic as corollaries to his proofs of the normalizability of natural deduction derivations. ${ }^{6}$ Our focus here will likewise be on (single-conclusion) natural deduction formulations of minimal, intuitionist, and classical propositional and first-order logics. (A couple of intermediate propositional logics will also be considered briefly. The intermediate first-order logic for constant domains - and its Kripke semantics-figures more prominently.) What novelty there is lies in the approach's being semantic, a fact that may be of pedagogical value.

When we present classical logic (propositional or first order) by adding schematic rules to a formulation of minimal or intuitionist logic — an extension-by-rules, as we might say, of minimal or intuitionist logic (propositional or first order) — we find that, in addition to subformulas of an invalid sequent, we may have to attend to certain other formulas too in order to obtain Kripke models of the appropriate kind. Exactly which formulas depends on the additional rules - exactly how the propositional part of classical logic is formulated becomes crucial. Nevertheless we can still obtain separation results when the subformula property fails. We obtain the strict subformula property for a particular formulation of classical propositional logic. However, even separation can only be obtained at the expense

4 Prawitz (1965, p. 16) gives something very close to this inductive definition:

(1) $A$ is a subformula of $A$;

(2a) If $B \wedge C, B \vee C$, or $B \rightarrow C$ is a subformula of $A$, then so are $B$ and $C$;

(2b) If $\neg B$ is a subformula of $A$, then so is $B$;

(3) If $\forall v B$ or $\exists v B$ is a subformula of $A$, then so is $B(v / c)$.

Here $B(v / c)$ represents the outcome of dropping the quantifier at the head of $\forall v B$ or $\exists v B$ and uniformly substituting the constant $c$ for all occurrences of the variable $v$ bound by the dropped quantifier. (In what follows we eschew function symbols and identity. We choose to formulate our natural deduction systems without free-variable formulas.) Prawitz's notion of subformula coincides with what Troelstra \& Schwichtenberg (1996, p. 4) call Gentzen subformula.

5 See, for example, Troelstra \& Schwichtenberg (1996, secs. 4.1 and 4.2). Classical propositional and first-order logics differ in their sequent calculus formulation from their intuitionist correlates only by a structural feature, namely the possibility that the succeedent contain two or more formulas. Sequent calculus formulations of these logics also enjoy the subformula and separation properties (again see, e.g., Troelstra \& Schwichtenberg, 1996). The case of the natural deduction formulations of these logics is much less straightforward-a matter we take up below.

6 See Prawitz (1965, secs. 3.1, 3.2, 4.1, and 4.2). Prawitz's results have subsequently been strengthened. See, for example, Stålmarck (1991). Below we obtain an optimal strengthening. 
of redundancy: we must take as basic rules obtainable as derived rules given the other rules present. That outcome is, however, predicated on formulating classical propositional logic by adding rules to intuitionist propositional logic. In that setting, redundancy can only be avoided either by taking $\neg A$ to abbreviate $A \rightarrow \perp$, where $\perp$ is the familiar 0 -ary falsum constant (rather than treating negation as a connective in its own right), or by taking $A \rightarrow B$ to be merely an abbreviation for $\neg A \vee B$.

However, taking both negation and implication as primitive we succeed in finding a natural deduction formulation of classical propositional logic in which all rules governing connectives are pure rules (i.e., each concerns only one connective), in which no rule is redundant, and that has the (strict) subformula (hence the separation) property. It is not an extension-by-rules even of minimal propositional logic as the standard introduction rule for negation is absent and the standard introduction rule for the conditional is severely restricted. The Rule of Dilemma governs negation; Tarski's Rule (as I call it), a close relative of Peirce's Rule, governs the conditional.

The first-order version of that formulation of classical logic, obtained by adding the orthodox introduction and elimination rules for the universal and existential quantifiers, does not have the subformula property, nor even the separation property-unsurprisingly, for the "constant domains" inference pattern $\forall x(F x \vee A) \vdash \forall x F x \vee A$, where $x$ does not occur free in $A$, is classically but not intuitionistically valid. To obtain a formulation of classical first-order logic satisfying the strict subformula property (and doing so without redundancy), we have to weaken the restrictions customarily imposed on applications of the standard introduction rule for the universal quantifier, and, to compensate, add restrictions on the use of $\vee$-elimination, Dilemma, and Tarski's Rule. In well-defined circumstances we allow generalization on a name that does occur in assumptions on which the instance generalized upon depends. Very roughly, inferring a universal generalization is permissible (classically!) even when the name does occur in undischarged assumptions if those assumptions containing the name are assumptions for the subproofs in applications of $\vee$-elimination, Dilemma, and Tarski's Rule, and, all importantly, in the case of $\checkmark$-elimination have an ancestry that can be traced back to assumptions not containing those names. Unfortunately, the completeness proof for this formulation of first-order classical logic is the one place where I have been unable to find an application for the general technique deployed elsewhere in this article. I do not know whether this is due merely to lack of imagination and ingenuity on my part or whether there is a deeper cause, but the complexity of extant completeness proofs for the constant domains extension of first-order intuitionist logic_-Görnemann (1971), Gabbay (1981)—suggests the latter.

In what follows we begin with a natural deduction formulation of intuitionist propositional and first-order logics with negation as primitive. We have then the standard introduction and elimination rules for $\wedge, \vee, \rightarrow, \neg, \forall$, and $\exists$.
$\wedge$-I $\frac{A \quad B}{A \wedge B}$
^-E $\frac{A \wedge B}{A}$
$\frac{A \wedge B}{B}$
$\checkmark-\mathrm{I}$
$\vee-E$

\begin{tabular}{ccc} 
& {$[A]$} & {$[B]$} \\
$A \vee B$ & $\vdots$ & $\vdots$ \\
\hline & $C$ & $C$ \\
\hline &
\end{tabular}




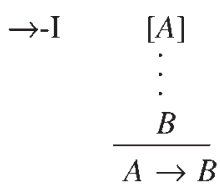

$\forall-\mathrm{I}$

$$
\frac{A}{\forall v A(c / v)}
$$

subject to the restriction

that $c$ not occur in any

assumption on which $A$ depends
$\frac{A \quad A \rightarrow B}{B}$

$\forall-\mathrm{E}$

$\frac{\forall v A}{A(v / c)}$

$\exists-\mathrm{I}$

$\exists-\mathrm{E}$

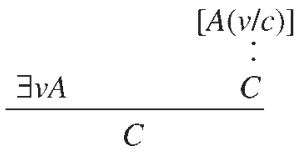

subject to the restrictions that $c$ not occur in $C$ nor in any assumption

$\frac{A(v / c)}{\exists v A}$

$C$

We take the standard rules for negation to be:
$\neg-I$

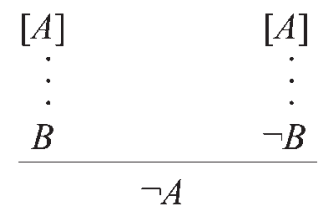
$\neg-\mathrm{E}$

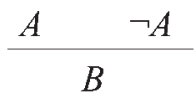

For certain purposes it is useful to think of $\neg$-I as derived from these two more primitive rules:
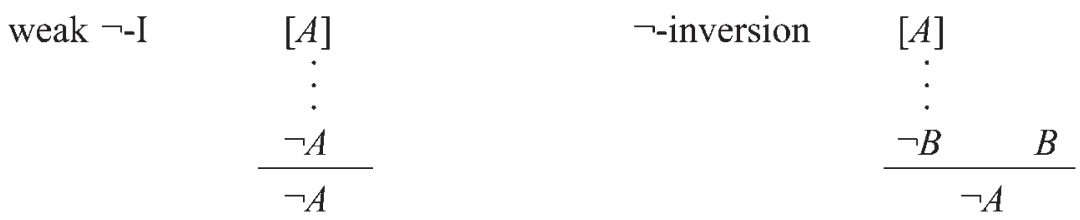

With our theme in mind, it is a significant, if obvious, fact that obtaining $\neg$-I as a derived rule from weak $\neg$-I and $\neg$-inversion (henceforth, $\neg$-inv) introduces no new formula:

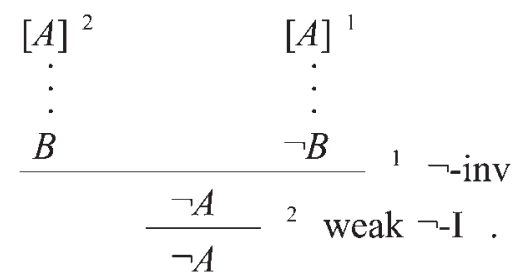

Minimal propositional logic may be obtained from intuitionist propositional logic by dropping the $\neg$-E rule.

That a set of natural deduction rules should enjoy the subformula and separation properties is undoubtedly a boon where the practical aim of constructing (or searching for) derivations is concerned and this paper concerns itself solely with the technical matter of 
the derivation of, for the most part, known proof-theoretic results by a semantic technique. Nevertheless, a very brief word ought, perhaps, to be said regarding the philosophical import of these formal results. It is conceptually an important matter that one's chosen set of rules of inference enjoy the separation property should one take the line that these rules give the meaning of the connectives, for only if the chosen rules enjoy the separation property can one go on to declare validity to turn on nothing other than the meanings of the connectives involved. And in that setting the subformula property takes on the guise of something very like a compositionality principle.

These all too cryptic observations will perforce suffice, for our present purpose is to sketch and apply a simple semantic method with which to investigate to what extent various sets of natural deduction rules do enjoy the separation and subformula properties.

\section{§2. Propositional logics.}

2.1. The basic Lindenbaum construction. The Lindenbaum construction for classical propositional logic yields a maximal, consistent, deductively closed set of formulas. In the classical case, maximality implies negation- and implication-completeness: for any formulas $A$ and $B$ in the language, the maximal set contains exactly one of $A$ and $\neg A$, and at least one of $A$ and $A \rightarrow B$. For weaker logics, maximality may not entail these properties. The set produced by the construction is always deductively closed-a theory. Which properties it possesses depends closely on which rules of inference, whether as primitive or derived, are available. For example, $\neg$-E ensures consistency.

Compared to the standard construction there is in what follows a double relativization. Given a sequent $\Sigma: A$ underivable in some logic, we confine attention to theories containing only formulas in some set determined by $\Sigma \cup\{A\}$, a set that includes at least all subformulas of $A$ and the formulas in $\Sigma$. The second relativization is to some not necessarily proper superset of this set of formulas. These are the formulas that are allowed to turn up in inferences-we restrict application of the rules of the logic in question to formulas in this second set.

We suppose given a language $\mathscr{L}$ comprising a set $\operatorname{At}(\mathscr{L})$ of atomic formulas and complex formulas built up using the connectives $\wedge, \vee, \rightarrow$, and $\neg$. We say that a connective occurs in a set of formulas just in case it occurs in at least one member of the set.

Given a set $\Delta$ of formulas of $\mathscr{L}$ and a set $R$ of rules of inference we have the following definitions:

(i) a set of formulas $\Gamma$ is a $\Delta, R$-theory if

$$
\text { for all formulas } A \text { in } \Delta, A \in \Gamma \text { if, and only if, } \Gamma \vdash_{R} A
$$

(where $\vdash_{R}$ stands for derivability governed by the rules in the set $R$ ) ${ }^{7}$

(ii) a set of formulas $\Gamma$ is $\Delta$-prime if

for all formulas $A, B$ in $\Delta$, if $A \vee B \in \Delta$ then $A \vee B \in \Gamma$ if, and only if, $A \in \Gamma$ or $B \in \Gamma$;

(iii) a set of formulas $\Gamma$ is $\Delta$-consistent if

for all formulas $A$ in $\Delta$, if $\neg A \in \Delta$ then $\neg A \in \Gamma$ only if $A \notin \Gamma$;

7 Notice that by this definition a $\Delta, R$-theory may contain formulas not in $\Delta$. 
(iv) a set of formulas $\Gamma$ is $\Delta$-negation-complete if

for all formulas $A$ in $\Delta$, if $\neg A \in \Delta$ and $A \notin \Gamma$ then $\neg A \in \Gamma$;

(v) a set of formulas $\Gamma$ is $\Delta$-implication-complete if

for all formulas $A, B$ in $\Delta$, if $A \rightarrow B \in \Delta$ and $A \notin \Gamma$ then $A \rightarrow B \in \Gamma{ }^{8}$

2.1.1. The Generic Lindenbaum Lemma (GLL) In what follows, we take for granted that all rules of inference, and proofs constructed in accordance with them, are finitary. We also take for granted that any set of rules considered allows us to derive a formula from any set containing that formula. We must allow that some rules of inference may be derived rules. Here a little care is needed. I shall speak of a derived rule being "safely $\Delta$-derived", where $\Delta$ is a set of sentences. In this there is implicit reference to some set of primitive rules $R$. What is meant is that in the application or applications in question of the derived rule to formulas in $\Delta$, there need be no application of rules in $R$ to formulas outside $\Delta$ when the derived rule is expanded in terms of the primitive rules.

THEOREM 1 Given $R$, when $\Sigma \nvdash_{R}$ A let $\Delta_{0}$ and $\Delta$ be sets of formulas such that $\Sigma \cup\{A\}$ $\subseteq \Delta_{0}$ and $\Delta_{0}$ is closed under subformulas (i.e., any subformula of a member of $\Delta_{0}$ belongs to $\left.\Delta_{0}\right), \Delta_{0} \subseteq \Delta$, and let $R \backslash \Delta$ stand for the restriction of the rules in $R$ obtained by excluding rules governing those connectives that do not occur in any member of $\Delta$ and restricting those that remain to formulas in $\Delta$, that is, only formulas in $\Delta$ may occur as assumptions, major and minor premises, and/or conclusions in applications of these rules, hence only formulas in $\Delta$ may occur in proofs constructed using the rules in $R \backslash \Delta$. Then

(i) there is a $\Delta_{0}, R \backslash \Delta$-theory $\Gamma$ such that $\Sigma \subseteq \Gamma \subseteq \Delta_{0}, A \notin \Gamma$, and

(a) for all formulas $B$ in $\Delta_{0}, B \notin \Gamma$ if, and only if, $\Gamma, B \vdash_{R \backslash \Delta} A$,

(b) if $\wedge$ occurs in $\Delta_{0}$ and the rules of $\wedge-I$ and $\wedge-E$ either belong to $R$ or are safely $\Delta$-derived rules given the rules in $R$, then for all formulas $B, C$ in $\Delta_{0}$, if $B \wedge C \in \Delta_{0}$ then $B \wedge C \in \Gamma$ if, and only if, $B \in \Gamma$ and $C \in \Gamma$,

(c) if $\rightarrow$ occurs in $\Delta_{0}$ and the rule of $\rightarrow-I$ either belongs to $R$ or is a safely $\Delta$ derived rule given the rules in $R$, then for all formulas $B, C$ in $\Delta_{0}$, if $B \rightarrow$ $C \in \Delta_{0}$ then $B \rightarrow C \in \Gamma$ if $C \in \Gamma$,

(d) if $\rightarrow$ occurs in $\Delta_{0}$ and the rule of $\rightarrow-E$ either belongs to $R$ or is a safely $\Delta$ derived rule given the rules in $R$, then for all formulas $B, C$ in $\Delta_{0}$, if $B \rightarrow$ $C \in \Delta_{0}$ then $B \rightarrow C \in \Gamma$ only if $B \notin \Gamma$ or $C \in \Gamma$;

(ii) if $\vee$ occurs in $\Delta_{0}$ and the rules of $\vee-I$ and $\vee-E$ either belong to $R$ or are safely $\Delta$-derived rules given the rules in $R, \Gamma$ is $\Delta_{0}$-prime;

(iii) if $\neg$ occurs in $\Delta_{0}$ and the rule of $\neg-E$ either belongs to $R$ or is a safely $\Delta$-derived rule given the rules in $R, \Gamma$ is $\Delta_{0}$-consistent;

(iv $\left.v_{1}\right)$ if $A=\neg D$, for some $D \in \Delta_{0}$ and the rule $\neg$-inv either belongs to $R$ or is a safely $\Delta$-derived rule given the rules in $R, \Gamma$ is $\Delta_{0}$-consistent;

(iv $v_{2}$ if $A=\neg D$, for some $D \in \Delta_{0}$ and the rule of $\neg$-I either belongs to $R$ or is a safely $\Delta$-derived rule given the rules in $R, \Gamma$ is $\Delta_{0}$-consistent and $\Delta_{0}$-negation-complete;

8 By these definitions, if $\vee$ does not occur in $\Delta$ any set of formulas is $\Delta$-prime; likewise, if $\neg$ does not occur in $\Delta$ any set of formulas is $\Delta$-consistent and $\Delta$-negation-complete, and if $\rightarrow$ does not occur in $\Delta$ any set of formulas is $\Delta$-implication-complete. 
(v) if $A=\neg D$, for some $D \in \Delta_{0}, \rightarrow$ occurs in $\Delta_{0}$ and the rules of weak $\neg-I, \neg-E$, and $\rightarrow-I$ either belong to $R$ or are safely $\Delta$-derived rules given the rules in $R, \Gamma$ is $\Delta_{0}$-implication-complete.

Proof. The proof mimics the standard version.

Let $B_{0}, B_{1}, \ldots, B_{n}, \ldots$ be an enumeration of the formulas in $\Delta_{0}$. This enumeration is indexed either by $\mathbb{N}$ or an initial segment of $\mathbb{N}$-call the indexing set $I$. We define a sequence of extensions of $\Sigma$ in the standard way:

(A) $\Gamma_{0}=\Sigma$;

(B) for all $n \in I$,

$\Gamma_{n+1}=\Gamma_{n}$ if $\Gamma_{n}, B_{n} \vdash_{R \backslash \Delta} A$,

$\Gamma_{n+1}=\Gamma_{n} \cup\left\{B_{n}\right\}$ if $\Gamma_{n}, B_{n} \nvdash_{R \mid \Delta} A$.

We set $\Gamma=\Gamma_{0} \cup \bigcup_{n \in I} \Gamma_{n+1}$.

By construction, we have, for all $n \in I$, that $\Gamma_{n+1} \nvdash_{R \mid \Delta} A$. As proofs are finitary it follows that $\Gamma \nvdash_{R \backslash \Delta} A$.

(i) If $B \in \Delta_{0}$ and $B \notin \Gamma$ then, for some $n \in I, B=B_{n}$, and $B \notin \Gamma_{n+1}$; but then $\Gamma_{n}, B \vdash_{R \mid \Delta} A$ and hence $\Gamma, B \vdash_{R \mid \Delta} A$. If $B \in \Delta_{0}, B \in \Gamma$ and $\Gamma, B \vdash_{R \mid \Delta} A$, then $\Gamma \vdash_{R \mid \Delta} A$, which we know not to be the case, hence $\Gamma, B \nvdash_{R \mid \Delta} A$ when $B \in \Delta_{0}$ and $B \in \Gamma$. As $\Gamma \vdash_{R \mid \Delta} A$ when $A \in \Gamma, A \notin \Gamma$.

$\Gamma$ is a $\Delta_{0}, R \backslash \Delta$-theory: if $B \in \Gamma$ then $\Gamma \vdash_{R \backslash \Delta} B$; conversely, if $\Gamma \vdash_{R \backslash \Delta} B, B \in \Delta_{0}$ and $B \notin \Gamma$ then $\Gamma \vdash_{R \mid \Delta} A$ as $\Gamma, B \vdash_{R \backslash \Delta} A$, but we know this not to be the case.

$(b),(c)$, and $(d)$ are straightforward consequences of the $\Delta_{0}, R \backslash \Delta$-theoryhood of $\Gamma$. These properties are therefore enjoyed by any $\Delta_{0}, R\lceil\Delta$-theory.

(ii) If $\vee$ occurs in $\Delta_{0}$ and $R$ gives us the rules of $\vee$-I and $\vee-\mathrm{E}, \Gamma$ is $\Delta_{0}$-prime: firstly, if $B, C, B \vee C \in \Delta_{0}$ and $B \in \Gamma$ or $C \in \Gamma$, then, by $\vee$-I, $\Gamma \vdash_{R \mid \Delta} B \vee C$ and, by $\Delta_{0}, R \backslash \Delta$-theoryhood of $\Gamma, B \vee C \in \Gamma$; secondly, if $B, C, B \vee C \in \Delta_{0}, B \notin \Gamma$ and $C \notin \Gamma$, then $\Gamma, B \vdash_{R \mid \Delta} A$ and $\Gamma, C \vdash_{R \mid \Delta} A$, whence, by $\vee$-E, $\Gamma, B \vee C \vdash_{R \mid \Delta} A$, and so $B \vee C \notin \Gamma$.

(iii) If $\neg$ occurs in $\Delta_{0}$ and $R$ gives us the rule of $\neg-\mathrm{E}, \Gamma$ is $\Delta_{0}$-consistent: if $B \in \Delta_{0}$, $\neg B \in \Delta_{0}, B \in \Gamma$, and $\neg B \in \Gamma$ then, by $\neg$-E, $\Gamma \vdash_{R \backslash \Delta} A$, which we know not to be the case.

(iv) If $A=\neg D$, and $R$ gives us the rule $\neg$-inv, $\Gamma$ is $\Delta_{0}$-consistent; if, in addition, $R$ gives us weak $\neg$-I, $\Gamma$ is also $\Delta_{0}$-negation-complete: firstly, if $B \in \Delta_{0}, \neg B \in \Delta_{0}$, $B \in \Gamma$, and $\neg B \in \Gamma$ then, by $\neg$-inv, $\Gamma \vdash_{R \mid \Delta} \neg D(=A)$, which we know not to be the case; secondly, if $B \in \Delta_{0}, \neg B \in \Delta_{0}, B \notin \Gamma$, and $\neg B \notin \Gamma$ then $\Gamma, B \vdash_{R \mid \Delta} \neg D$ and $\Gamma, \neg B \vdash_{R \mid \Delta} \neg D$, whence, from the former, by $\neg$-inv, $\Gamma, D \vdash_{R \mid \Delta} \neg B$, and so, from this and the latter, $\Gamma, D \vdash_{R \backslash \Delta} \neg D$, yielding, by weak $\neg-\mathrm{I}, \Gamma \vdash_{R \backslash \Delta} \neg D(=A)$, which we know not to be the case.

(v) If $A=\neg D, \rightarrow$ occurs in $\Delta_{0}$, and $R$ gives us the rules of weak $\neg$-I, $\neg$-E, and $\rightarrow$-I then $\Gamma$ is $\Delta_{0}$-implication-complete: if $B, C, B \rightarrow C \in \Delta_{0}, B \notin \Gamma$, and $B \rightarrow C \notin \Gamma$, then $\Gamma, B \vdash_{R \mid \Delta} \neg D$ and $\Gamma, B \rightarrow C \vdash_{R \mid \Delta} \neg D$, whence, from the former, by $\neg$-E, $\Gamma, B, D \vdash_{R \backslash \Delta} C$, and so, by $\rightarrow-\mathrm{I}, \Gamma, D \vdash_{R \backslash \Delta} B \rightarrow C$ and from this and the latter we have $\Gamma, D \vdash_{R \backslash \Delta} \neg D$, yielding, by weak $\neg-\mathrm{I}, \Gamma \vdash_{R \backslash \Delta} \neg D(=A)$, which we know not to be the case.

With $\Gamma$ and $\Delta_{0}$ as above and the antecedents of (i) $(a)$ - $(d)$ fulfilled, a $\Delta_{0}$-consistent, $\Delta_{0}$-prime, $\Delta_{0}$-negation-complete, $\Delta_{0}$-implication-complete $\Delta_{0}, R$-theory $\Gamma$ corresponds 
to a classical assignment of truth-values to the formulas in $\Delta_{0}$ under which all and only members of $\Gamma$ receive the value true.

The proof allows that $\Delta_{0}$ may contain more than subformulas of members of $\Sigma \cup\{A\}$ and that $\Delta$ may contain formulas not in $\Delta_{0}$. When we wish to indicate that some set $R$ of rules is to be restricted in application exactly to subformulas of members of some set $\boldsymbol{\Phi}$, we shall lazily write $R\lceil\Phi$ rather than coin a special notation for the least set containing $\boldsymbol{\Phi}$ and closed under subformulas.

2.1.2. Strong Glivenko Theorems for classical propositional logic (CPL) Independently of any particular formulation of classical propositional logic as an extension of minimal propositional logic (MPL), for the first, and intuitionist propositional logic (IPL), for the second, we have, as immediate consequences of GLL, the following, where $R M$ denotes the set of rules in our chosen formulation of MPL, and, likewise, $R I$ denotes the set of rules in our chosen formulation of IPL. Given a set of formulas $\Delta$ we write $C(\Delta)$ for the set of connectives that occur in at least one member of $\Delta$.

\subsubsection{Strong Glivenko Theorem for the $\{\wedge, \vee, \neg\}$-fragment of $C P L$}

THEOREM 2 If $C(\Sigma \cup\{\neg A\}) \subseteq\{\wedge, \vee, \neg\}$ and $\Sigma \vdash_{\mathrm{CPL}} \neg A$ then $\Sigma \vdash_{\mathrm{MPL}} \neg A$.

Proof. Let $\Delta$ contain all and only subformulas of the members of $\Sigma \cup\{\neg A\}$. $\neg$-I is one of the rules of MPL, so if $\Sigma \nvdash_{\text {MPL }} \neg A$, there is, by GLL (i)-(iv), a $\Delta$-prime, $\Delta$-consistent, $\Delta$-negation-complete, vacuously $\Delta$-implication-complete, $\Delta, R M \backslash \Delta$-theory $\Gamma$, a subset of $\Delta$, extending $\Sigma$ and not containing $\neg A$. This determines a classical assignment of truthvalues under which all members of $\Sigma$ are assigned the value true and $\neg A$ is assigned the value false. By the soundness of CPL with respect to classical truth-value assignments, $\Sigma \nvdash_{\mathrm{CPL}} \neg A$.

\subsubsection{Strong Glivenko Theorem for full CPL}

\section{THEOREM 3 If $\Sigma \vdash_{\mathrm{CPL}} \neg A$ then $\Sigma \vdash_{\mathrm{IPL}} \neg A$.}

Proof. $\rightarrow-$ I and $\neg$-E are among the rules of IPL, so, over and above what we appealed to in the proof of the previous theorem, by GLL (v) the $R I \backslash \Delta$-theory $\Gamma$ is $\Delta$-implicationcomplete.

\subsection{Intuitionist propositional logic (IPL).}

2.2.1. Kripke semantics for intuitionist propositional logic A Kripke model is a triple $<T, \preceq, u>$ where

(a) $T$ is a nonempty set (whose elements are called nodes);

(b) $\preceq \subseteq T^{2}$ and partially orders $T$;

(c) the function $u: T \times A t(\mathscr{L}) \rightarrow\{0,1\}$ satisfies the constraint: if $u_{s}(A)=1$ and $s \preceq t$ then $u_{t}(A)=1$.

The function $v: T \times \mathscr{L} \rightarrow\{0,1\}$ extends $u$ in the following ways:

(d) for all $A \in A t(\mathscr{L})$ and $t \in T, v_{t}(A)=u_{t}(A)$;

(e) for all $A, B \in \mathscr{L}, v_{t}(A \wedge B)=\min \left\{v_{t}(A), v_{t}(B)\right\}$;

(f) for all $A, B \in \mathscr{L}, v_{t}(A \vee B)=\max \left\{v_{t}(A), v_{t}(B)\right\}$;

(g) for all $A, B \in \mathscr{L}, v_{t}(A \rightarrow B)=1$ iff $\forall s \succeq t\left[v_{s}(A)=0\right.$ or $\left.v_{s}(B)=1\right]$;

(h) for all $A \in \mathscr{L}, v_{t}(\neg A)=1$ iff $\forall s \succeq t\left[v_{s}(A)=0\right]$. 
We say that a formula $A$ is verified at a node $t$ in a model $<T, \preceq, u>$ just in case $v_{t}(A)=1$.

A model $<T, \preceq, u>$ contains a base node if there is a node $t \in T$ such that, for all $s \in T, s \succeq t$.

Soundness of IPL: if $\Sigma \vdash_{\text {IPL }} A$ then at any node in a Kripke model $<T, \preceq, u>$ at which every member of $\Sigma$ is verified $A$ is verified there too.

We note in passing that in the terminology of 2.1.1, the set of formulas verified at a node in a Kripke model constitutes an $\mathscr{L}$-consistent, $\mathscr{L}$-prime $\mathscr{L}, R I(\mathscr{L})$-theory, that is, a consistent, prime theory on the orthodox reading of these terms.

\subsubsection{Generic completeness theorem}

THEOREM 4 If $\Sigma \nvdash_{R I \uparrow(\Sigma \cup\{A\})}$ A then there is a Kripke model $\langle T, \preceq, u>$ with a base node such that all members of $\Sigma$ are verified at the base node but $A$ is not verified there.

Proof. We take $\Delta$ to be the least subset of $\mathscr{L}$ extending $\Sigma \cup\{A\}$ and closed under subformulas. (We have immediately that $C(\Delta)=C(\Sigma \cup\{A\})$ and that $R I \backslash \Delta=R I \uparrow(\Sigma \cup\{A\})$.) Clauses (i), (ii), and (iii) of the Generic Lindenbaum Lemma (GLL) apply so there is a $\Delta$-consistent, $\Delta$-prime $\Delta, R I \backslash \Delta$-theory $\Gamma$ contained in $\Delta$, extending $\Sigma$ and not containing $A$ with the properties stated in (i) $(a)-(d)$.

The nodes of our small Kripke model are those subsets of $\Delta$ extending $\Gamma$ that are $\Delta$ consistent, $\Delta$-prime $\Delta, R I \uparrow \Delta$-theories. ${ }^{9} \Gamma$ is the base node of the model. $\preceq$ is the subset relation. We stipulate that the atomic formula $B$ is verified at the $\Delta, R I \uparrow \Delta$-node $\Theta$ just in case $B \in \Theta$.

Claim For all $\Delta, R I \uparrow \Delta$-theory-nodes $\Theta$ in the model and all $B \in \Delta, B$ is verified at $\Theta$ just in case $B \in \Theta$.

As ever, proof is by induction on complexity of formula (number of occurrences of connectives).

The claim holds by stipulation for atomic formulas.

The induction hypothesis states that the claim holds for all formulas in $\Delta$ up to some given complexity. We consider the next higher level of complexity.

Induction step. Cases:

Conjunction If $B \wedge C \in \Delta$ then the rules of $\wedge$-I and $\wedge$-E belong to $R I \uparrow \Delta$. We then have that

$B \wedge C \in \Theta$ iff $B \in \Theta$ and $C \in \Theta$ (by GLL (i) $(b)$ ), iff $v_{\Theta}(B)=1$ and $v_{\Theta}(C)=1$ (by the induction hypothesis), iff $v_{\Theta}(B \wedge C)=1$ (by definition of $v$ ).

Disjunction If $B \vee C \in \Delta$ then the rules of $\vee$-I and $\vee$-E, restricted to formulas in $\Delta$, belong to $R I \uparrow \Delta$ and we have that

$B \vee C \in \Theta$ iff $B \in \Theta$ or $C \in \Theta$ (by $\Delta$-primeness of $\Theta$ ), iff $v_{\Theta}(B)=1$ or $v_{\Theta}(C)=1$ (by the induction hypothesis), iff $v_{\Theta}(B \vee C)=1$ (by definition of $v$ ).

Implication If $B \rightarrow C \in \Delta$ then the rules of $\rightarrow-\mathrm{I}$ and $\rightarrow$-E belong to $R I \uparrow \Delta$.

If $B \rightarrow C \in \Theta$ then, for all $\Delta, R I \backslash \Delta$-theories $\Xi$ extending $\Theta, B \rightarrow C \in \Xi$; by the induction hypothesis, if $v_{\Xi}(B)=1, B \in \Xi$; as $B \rightarrow C \in \Xi$, by GLL (i) (d), $C \in \Xi$; by another appeal to the induction hypothesis, $v_{\Xi}(C)=1$; so we have that $\forall \Xi \supseteq \Theta\left[v_{\Xi}(B)=0\right.$ or $\left.v_{\Xi}(C)=1\right]$, that is, $v_{\Theta}(B \rightarrow C)=1$.

If $B \rightarrow C \notin \Theta$ then, by $\Delta, R I \backslash \Delta$-theoremhood of $\Theta, \Theta \nvdash_{R I \mid \Delta} B \rightarrow C$, in which case, $\Theta, B \nvdash_{R I \mid \Delta} C$ (as follows by considering $\rightarrow$-I restricted to formulas

9 Every $\Delta, R I \backslash \Delta$-theory is an extension of itself. 
in $\Delta$ ); by GLL, there is a $\Delta, R I \uparrow \Delta$-theory $\Xi$ satisfying GLL (i)-(iii) and such that $\Theta \cup\{B\} \subseteq \Xi \subseteq \Delta$ and $C \notin \Xi$; by the induction hypothesis, $v_{\Xi}(B)=1$ and $v_{\Xi}(C)=0$, whence $v_{\Theta}(B \rightarrow C)=0$.

Negation If $\neg B \in \Delta$ then the rules of $\neg$-I and $\neg$-E, restricted to formulas in $\Delta$, belong to $R I \uparrow \Delta$ and we have that

If $\neg B \in \Theta$ then, for all $\Delta, R I \uparrow \Delta$-theories $\Xi$ extending $\Theta, \neg B \in \Xi$; as the $\Delta, R I \uparrow \Delta$-theories in our model are all $\Delta$-consistent, none of the $\Delta, R I \uparrow \Delta$-theories of interest extending $\Theta$ contains $B$; by the induction hypothesis we have that $\forall \Xi \supseteq \Theta\left[v_{\Xi}(B)=0\right]$, that is, $v_{\Theta}(\neg B)=1$.

If $\neg B \notin \Theta$ then, by $\Delta, R I \backslash \Delta$-theoremhood of $\Theta, \Theta \nvdash_{R I \backslash \Delta} \neg B$, in which case $\Theta, B \nvdash_{R I \uparrow \Delta} \neg B$ (as follows by considering weak $\neg$-I restricted to formulas in $\Delta$ ); by the GLL, there is a $\Delta, R I \backslash \Delta$-theory $\Xi$ satisfying GLL (i)-(iii) and such that $\Theta \cup\{B\} \subseteq \Xi \subseteq \Delta$ (and $\neg B \notin \Xi$ ); by the induction hypothesis, $v_{\Xi}(B)=1$, whence $v_{\Theta}(\neg B)=0$.

We have shown that the claim holds.

As $\Sigma \subseteq \Gamma$, all members of $\Sigma$ are verified at the base node, $\Gamma$, but $A$ is not.

2.2.3. Subformula and separation properties for intuitionist propositional logic As an immediate consequence of the soundness of IPL with respect to Kripke models and the previous result we have that IPL enjoys the subformula and separation properties when formulated using the standard natural deduction rules. Formally:

TheOrem 5 If $\Sigma \vdash_{\text {PPL }} A$ then $\Sigma \vdash_{R I(\Sigma \cup\{A\})} A$.

It is well known that none of the introduction or elimination rules of IPL is redundant. The quickest way to show this is this: give the other connectives their classical interpretation - their introduction and elimination rules are all sound; if showing an introduction rule nonredundant treat formulas with the connective in question dominant as uniformly false - the elimination rule is then classically sound but the introduction rule is not; if showing an elimination rule nonredundant treat formulas with the connective in question dominant as uniformly true-the introduction rule is then classically sound but the elimination rule is not.

\subsubsection{The smallness of small Kripke models}

2.2.4.1. Example $\neg \neg P \nvdash_{\mathrm{IPL}} P \quad$ Here $\Delta=\{P, \neg P, \neg \neg P\} . C(\Delta)=\{\neg\} . \Gamma=\{\neg \neg P\}$ and the only $\Delta$-consistent (and vacuously $\Delta$-prime) $\Delta, R I \backslash \Delta$-theory contained in $\Delta$ that properly extends $\Gamma$ is $\Theta=\{P, \neg \neg P\}$. We obtain the Kripke model Figure 1

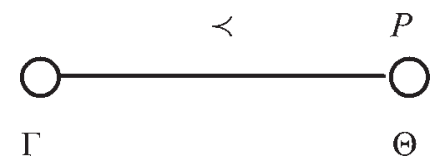

Fig. 1.

No atomic formula is verified at the node $\Gamma ; P$ is the only atomic formula verified at the node $\Theta$. This is the smallest possible Kripke model showing the failure of double negation elimination in IPL.

2.2.4.2. Example $\neg(P \wedge Q) \nvdash_{\mathrm{IPL}} \neg P \vee \neg Q \quad$ Here $\Delta=\{P, Q, \neg P, \neg Q, P \wedge Q$, $\neg P \vee \neg Q, \neg(P \wedge Q)\} . C(\Delta)=\{\wedge, \vee, \neg\} . \Gamma=\{\neg(P \wedge Q)\}$. There are three $\Delta$-consistent, $\Delta$-prime $\Delta, R I \uparrow \Delta$-theories contained in $\Delta$ that properly extend $\Gamma: \Theta_{1}=\{P, \neg Q, \neg P \vee \neg Q$, 
$\neg(P \wedge Q)\} ; \Theta_{2}=\{Q, \neg P, \neg P \vee \neg Q, \neg(P \wedge Q)\} ; \Theta_{3}=\{\neg P, \neg Q, \neg P \vee \neg Q, \neg(P \wedge Q)\}$. These give us the Kripke model Figure 2

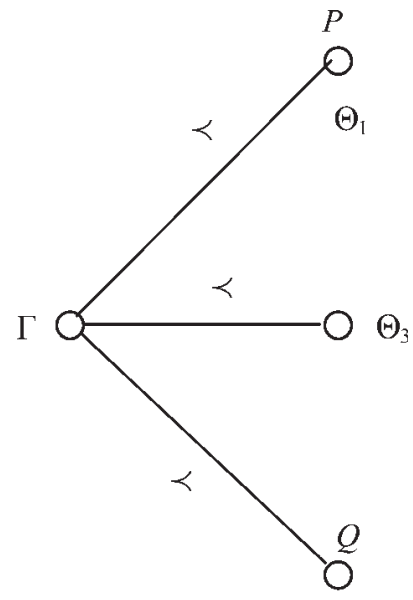

$\Theta_{2}$

Fig. 2 .

No atomic formulas are verified at the nodes $\Gamma$ and $\Theta_{3}$. The indicated atomic formulas, and only these, are verified at the nodes $\Theta_{1}$ and $\Theta_{2}$.

While certainly small this is not the smallest Kripke model showing failure of the De Morgan inference from $\neg(P \wedge Q)$ to $\neg P \vee \neg Q$ in IPL-the node $\Theta_{3}$ can be excised without loss.

2.2.5. An application of small Kripke models: the $\{\wedge, \vee, \rightarrow\}$-fragment of the intermediate logic $K C$ The intermediate propositional logic commonly known as KC but which I shall call KPL ${ }^{10}$ may be obtained by adding to IPL the rule

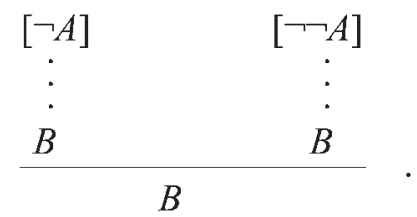

It is known to be sound and complete with respect to Kripke models for IPL satisfying this constraint on the ordering of nodes:

(K) $\forall t, s$ [if $\exists r(r \preceq t \& r \preceq s)$ then $\exists w(t \preceq w \& s \preceq w)] .^{11}$

THEOREM 6 If $C(\Sigma \cup\{A\}) \subseteq\{\wedge, \vee, \rightarrow\}$ and $\Sigma \vdash_{\mathrm{KPL}} A$ then $\Sigma \vdash_{\mathrm{IPL}} A$.

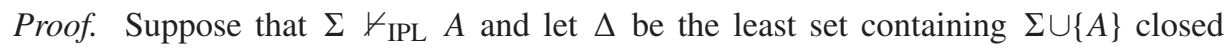
under subformulas. By the proof of Theorem 4 we know that there is a Kripke model with a base node $\Gamma$ at which all members of $\Sigma$ are verified but $A$ is not. The nodes in the model are exactly those subsets of $\Delta$ extending $\Gamma$ that satisfy GLL (i) and (ii), that is, they are exactly the $\Delta$-prime $\Delta, R I \uparrow \Delta$-theories extending $\Gamma$ and contained in $\Delta$. GLL

$\overline{10 \text { Segerberg }}(1968)$ calls it $I K$.

11 See, for example, Segerberg (1968, pp. 37-38). 
(iii) is satisfied vacuously. $\Delta$ itself is such a $\Delta$-prime $\Delta, R I \uparrow \Delta$-theory. Consequently, the constraint $(K)$ on the ordering of nodes is satisfied; by soundness of KPL, $\Sigma \nvdash_{\mathrm{KPL}} A$.

\subsection{Minimal propositional logic (MPL).}

2.3.1. Kripke semantics for minimal propositional logic A Kripke model for minimal propositional logic is a quadruple $<T, \preceq, u, \mathscr{F}>$ where

(a) $T$ is a nonempty set of nodes;

( $\left.\mathrm{b}_{1}\right) \preceq \subseteq T^{2}$ and partially orders $T$;

$\left(\mathrm{b}_{2}\right) \mathscr{F}$ is a possibly empty, not necessarily proper, subset of $T$ satisfying the constraint that if $s \in \mathscr{F}$ and $s \preceq t$ then $t \in \mathscr{F}$;

(c) the function $u: T \times A t(\mathscr{L}) \rightarrow\{0,1\}$ satisfies the constraint: if $u_{s}(A)=1$ and $s \preceq t$ then $u_{t}(A)=1$.

The function $v: T \times \mathscr{L} \rightarrow\{0,1\}$ extends $u$ in the same ways as for Kripke models for IPL with the exception of the clause dealing with negations. Here we have

$$
\left(\mathrm{h}^{\prime}\right) \text { for all } A \in \mathscr{L}, v_{t}(\neg A)=1 \text { iff } \forall s \succeq t\left[v_{s}(A)=0 \text { or } s \in \mathscr{F}\right] .
$$

As above, we say that a formula $A$ is verified at a node $t$ in a model $<T, \preceq, u, \mathscr{F}>$ just in case $v_{t}(A)=1$.

Soundness of MPL: if $\Sigma \vdash_{\text {MPL }} A$ then at any node in a Kripke model $<T, \preceq, u, \mathscr{F}>$ at which every member of $\Sigma$ is verified $A$ is verified there too.

We note that, in the terminology of 2.1.1, the set of formulas verified at a node in a Kripke model constitutes an $\mathscr{L}$-prime $\mathscr{L}, R M \backslash \mathscr{L}$-theory, that is, a prime theory on the orthodox reading of these terms. The set is $(\mathscr{L}-)$ consistent if, and only if, the node does not belong to $\mathscr{F}$.

\subsubsection{Completeness theorems}

\subsubsection{Generic completeness theorem}

THEOREM 7 If $\Sigma \nvdash_{R M \uparrow(\Sigma \cup\{A\})}$ A then there is a Kripke model $<T, \preceq, u, \mathscr{F}>$ with a base node such that all members of $\Sigma$ are verified at the base node but $A$ is not verified there.

Proof. As above, let $\Delta$ be the least subset of $\mathscr{L}$ extending $\Sigma \cup\{A\}$ and closed under subformulas. We have immediately that $C(\Delta)=C(\Sigma \cup\{A\})$ and that $R M \uparrow \Delta=R M \uparrow(\Sigma \cup$ $\{A\}$ ). Clauses (i) and (ii) of GLL apply so we have that there is a $\Delta$-prime $\Delta, R M \uparrow \Delta$-theory $\Gamma$ contained in $\Delta$, extending $\Sigma$ and not containing $A$. (As we no longer have the rule of $\neg$-E, we cannot guarantee that $\Gamma$ is $\Delta$-consistent when $\neg$ occurs in $\Delta$.)

The nodes of our Kripke model are those subsets of $\Delta$ that constitute $\Delta$-prime $\Delta, R M \backslash \Delta$ theories extending $\Gamma$. $\mathscr{F}$ is the set of $\Delta$-inconsistent, $\Delta$-prime, $\Delta, R M \uparrow \Delta$-theories in $\Delta$ extending $\Gamma$. $\Gamma$ is the base node of the model. $\preceq$ is the subset relation. We stipulate that the atomic formula $B$ is verified at the $\Delta, R M \uparrow \Delta$-theory-node $\Theta$ iff $B \in \Theta$.

Claim For all ( $\Delta$-prime) $\Delta, R M \uparrow \Delta$-theory-nodes $\Theta$ in the model and all $B \in \Delta, B$ is verified at $\Theta$ just in case $B \in \Theta$.

As before, proof is by induction on complexity of formula, the claim holding by stipulation for atomic formulas. Except for dropping mention of $\Delta$-consistency and writing $R M$ for $R I$, proof proceeds exactly as in Theorem 4 in the cases of conjunction, disjunction, and implication. The only interesting case in the induction step is, then, 
Negation If $\neg B \in \Delta$ then the rule of $\neg$-I, restricted to formulas in $\Delta$, belongs to $R M \uparrow \Delta$ and we have that

If $\neg B \in \Theta$ then, for all $\Delta, R M \backslash \Delta$-theories $\Xi$ extending $\Theta, \neg B \in \Xi$; if $v_{\Xi}(B)=1$ at the theory-node $\Xi$ then, by the induction hypothesis, $B \in \Xi$ and $\Xi$ is $\Delta$-inconsistent; so we find that $\forall \Xi \supseteq \Theta\left[v_{\Xi}(B)=0\right.$ or $\left.\Xi \in \mathscr{F}\right]$, that is, $v_{\Theta}(\neg B)=1$.

If $\neg B \notin \Theta$ then, by $\Delta, R M \uparrow \Delta$-theoremhood of $\Theta, \Theta \nvdash_{R M \uparrow \Delta} \neg B$, in which case $\Theta$, $B \nvdash R M \backslash \neg \neg$ (as follows by considering weak $\neg$-I restricted to formulas in $\Delta$ ); by the GLL, there is a $\Delta, R M \vdash \Delta$-theory $\Xi$ satisfying GLL (i) and (ii) and such that $\Theta \cup\{B\}$ $\subseteq \Xi \subseteq \Delta$ and $\neg B \notin \Xi$; we must show that $\Xi$ is $\Delta$-consistent: if it is not, then, for some formula $C \in \Delta$ such that $\neg C \in \Delta$, we have that $C \in \Xi$ and $\neg C \in \Xi$, but then, in virtue of the $\neg$-inv rule applied only to formulas in $\Delta, \Xi \vdash_{R M \vdash \Delta} \neg B$, and, by the $\Delta, R M \backslash \Delta$-theoryhood of $\Xi, \neg B \in \Xi$, which is not the case; we have that $\Xi \notin \mathscr{F}$ and, by the induction hypothesis, $v_{\Xi}(B)=1$, hence $v_{\Theta}(\neg B)=0 .{ }^{12}$

The claim therefore holds.

As $\Sigma \subseteq \Gamma$, all members of $\Sigma$ are verified at the base node, $\Gamma$, but $A$ is not.

\subsubsection{Subformula and separation properties for minimal propositional logic}

THEOREM 8 If $\Sigma \vdash_{\text {MPL }} A$ then $\Sigma \vdash_{R M \uparrow(\Sigma \cup\{A\})} A$.

Proof. Immediate by soundness of MPL with respect to the appropriate class of Kripke models and the previous result.

2.3.4. Example $\neg P \nvdash_{\mathrm{MPL}} P \rightarrow Q$ Here $\Delta=\{P, Q, \neg P, P \rightarrow Q\} . C(\Delta)=\{\neg, \rightarrow\}$. $\Gamma=\{P, \neg P\}$. The only (vacuously $\Delta$-prime) $\Delta, R M \backslash \Delta$-theory properly extending $\Gamma$ and contained in $\Delta$ is $\Delta$ itself. $\Gamma$ and $\Delta$ are both $\Delta$-inconsistent. So the nodes of our model are $\Gamma$ and $\Delta$ and both belong to $\mathscr{F}$. Our Kripke model is Figure 3

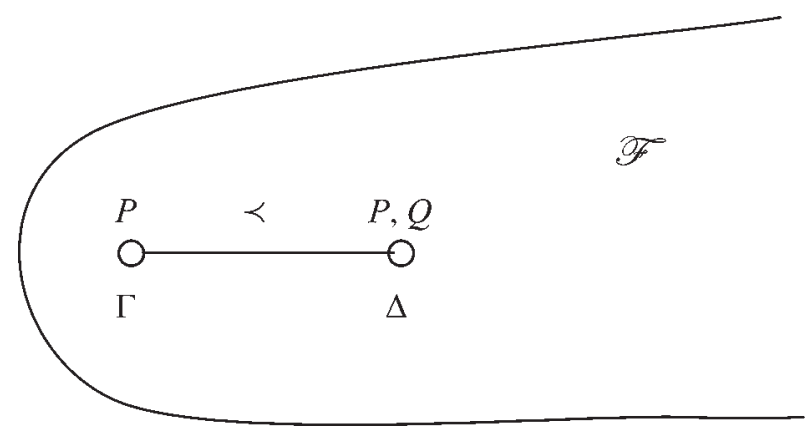

Fig. 3.

$P$ is the only atomic formula verified at $\Gamma$; both $P$ and $Q$ are verified at $\Delta$.

This is one of the smallest possible Kripke models showing the failure of the negative paradox of implication in MPL. However, it is perhaps not the most natural. Asked to produce a model showing its failure, one is, I think, much more likely to come up with this Figure 4

12 It's the last step here that requires an unavoidable use of full strength $\neg$-I in the absence of $\neg$-E. In the completeness theorem for IPL we used only of weak $\neg$-I, making no appeal to $\neg$-inv. Consequently, IPL can be formulated using weak $\neg-\mathrm{I}$ alone. Minimal propositional logic cannot, as was known by Wajsberg by 1939—-see Curry (1963, p. 285). 


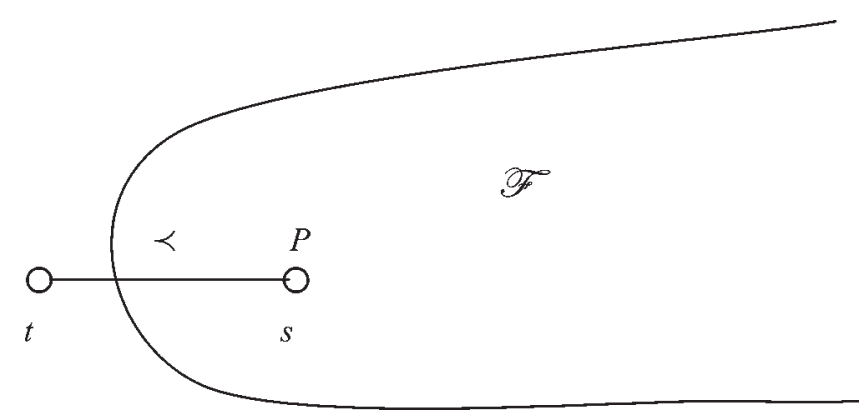

Fig. 4.

$P$ is the only atomic formula verified at $s$; no atomic formula is verified at the base node $t$.

2.4. Classical conjunction and disjunction. It is well known that the introduction and elimination rules for $\wedge$ and $\vee$ that we find in minimal and intuitionist propositional logic suffice to derive all classically valid sequents containing occurrences of only these connectives. That this is so falls out directly from the proof of completeness in 2.3.2.1 above.

2.4.1. Subformula and separation properties for the $\{\wedge, \vee\}$-fragment of classical propositional logic

TheOrem 9 If $\Sigma \vdash_{\mathrm{CPL}} A$ and $C(\Sigma \cup\{A\}) \subseteq\{\wedge, \vee\}$ then $\Sigma \vdash_{R M \uparrow(\Sigma \cup\{A\})} A$.

Proof. Suppose that $C(\Sigma \cup\{A\}) \subseteq\{\wedge, \vee\}$ and that $\Sigma \nvdash_{R M \uparrow(\Sigma \cup\{A\})} A$. Let $\Delta$ contain all and only subformulas of the members of $\Sigma \cup\{A\}$. By the GLL, there's a $\Delta$-prime, vacuously $\Delta$-consistent, vacuously $\Delta$-negation-complete, vacuously $\Delta$-implication-complete, $\Delta, R M \uparrow \Delta$-theory $\Gamma$, a subset of $\Delta$, extending $\Sigma$ and not containing $A$. As noted in 2.1.1 above, membership of $\Gamma$ maps to the value true under a classical assignment of truth-values to the formulas in $\Delta$ under which all and only the atomic formulas in $\Gamma$ are true: under this assignment all members of $\Sigma$ are true and $A$ is false.

\subsubsection{Example}

$$
P \vee Q \nvdash_{\mathrm{MPL}} P \wedge Q . \Delta=\{P, Q, P \wedge Q, P \vee Q\} ; C(\Delta)=\{\wedge, \vee\}
$$

Here there are two possible base nodes: $\Gamma_{1}=\{P, P \vee Q\} ; \Gamma_{2}=\{Q, P \vee Q\} . \Delta$ itself is the only $\Delta$-prime $\Delta, R M \uparrow \Delta$-theory in $\Delta$ extending these. $\Gamma_{1}$ and $\Gamma_{2}$ both generate classical valuations: the first has $P$ true, $Q$ false, the second the other way around. To labour the obvious, both make $P \vee Q$ true, $P \wedge Q$ false, as required.

The full Kripke model construction in 2.3.2.1 would give us a model with either of the two theories $\Gamma_{1}$ or $\Gamma_{2}$ as the base node and $\Delta$ as the other. As any classical valuation constitutes a one-node Kripke model we would again fail to obtain one of the smallest Kripke models showing the nonderivability of $P \vee Q \vdash P \wedge Q$ in MPL.

2.5. Classical negation. Before proceeding further we note the well-known fact that no formulation of CPL that adds either to MPL or IPL solely a pure rule dealing with negation such as those to be investigated immediately below-double negation elimination (DNE), classical reductio ad absurdum (CRA), Dilemma - has the separation property as various classically valid, intuitionistically invalid $\neg$-free sequents are derivable in CPL: for 
example, $(P \rightarrow Q) \rightarrow P \vdash P, \vdash(P \rightarrow Q) \vee(Q \rightarrow P),((P \rightarrow Q) \rightarrow Q) \wedge((Q \rightarrow$ $P) \rightarrow P) \vdash P \vee Q$.

\subsubsection{Double negation elimination}

2.5.1.1. CPL as MPL + DNE Perhaps the commonest way to obtain a natural deduction presentation of classical propositional logic (CPL) is to add the rule of double negation elimination (DNE) to minimal propositional logic (MPL). We write $R C \uparrow \Delta$ for the restriction of this set of rules to formulas in $\Delta$.

\subsection{Generic Lindenbaum Lemma: first extension}

THEOREM 10 Where $\Delta_{0}$ and $\Delta$ are sets of formulas such that $\Sigma \cup\{A\} \subseteq \Delta_{0} \subseteq \Delta$ and $\Delta_{0}$ is closed under subformulas,

(iv $v_{\mathrm{DNE}}$ ) provided $\Delta_{0} \cup\{\neg A, \neg \neg A\} \subseteq \Delta$, if $\neg$ occurs in $\Delta_{0}$ and the rules of $\neg-I$ and $D N E$ either belong to $R$ or are safely $\Delta$-derived rules given the rules in $R, \Gamma$ is $\Delta_{0^{-}}$ consistent and $\Delta_{0}$-negation-complete.

Proof. $\Delta_{0}$-consistency: if $B \in \Delta_{0}, \neg B \in \Delta_{0}, B \in \Gamma$, and $\neg B \in \Gamma$ then, by $\neg$-inv, $\Gamma \vdash_{R \backslash \Delta} \neg \neg A$ and hence, by DNE, $\Gamma \vdash_{R \backslash \Delta} A$, which we know not to be the case.

$\Delta_{0}$-negation-completeness: if $B \in \Delta_{0}, \neg B \in \Delta_{0}, B \notin \Gamma$, and $\neg B \notin \Gamma$ then $\Gamma, B \vdash_{R \vdash \Delta}$ $A$ and $\Gamma, \neg B \vdash_{R \backslash \Delta} A$; from the former, by $\neg-\mathrm{I} \Gamma, \neg A \vdash_{R \backslash \Delta} \neg B$, whence, with the second, $\Gamma, \neg A \vdash_{R \mid \Delta} A$; by $\neg$-I again, $\Gamma, \vdash_{R \mid \Delta} \neg \neg A$ and hence, by DNE, $\Gamma \vdash_{R \mid \Delta} A$, which we know not to be the case.

2.5.1.1.2. A weak subformula property for the $\{\wedge, \vee, \neg\}$-fragment of $C P L$ as $M P L+$ $D N E$

THEOREM 11 When $C P L=M P L+D N E$, the $\{\wedge, \vee, \neg\}$-fragment of classical propositional lacks the strict subformula property.

Proof. $\neg(P \wedge Q) \vdash \neg P \vee \neg Q$ is classically valid but not intuitionistically so, hence any classical derivation must employ DNE at least once. As no formula in $\{P, Q, \neg P, \neg Q$, $P \wedge Q, \neg P \vee \neg Q, \neg(P \wedge Q)\}$ is headed by ' $\neg \neg$ ', the subformula property fails. ${ }^{13}$

However, Theorem 10 in conjunction with what has already been established tells us that in the derivation of any $\rightarrow$-free sequent $\Sigma \vdash A$ that is classically valid but not derivable in MPL, only subformulas of members of $\Sigma \cup\{\neg \neg A\}$ need occur. As a corollary to Theorem 2 (Strong Glivenko Theorem) we have that DNE, which must be used in deriving the sequent, need be used only once and we may further insist that its application be the last step. Natural deduction proofs complying with that last constraint can be somewhat indirectwitness derivation of $\neg \neg P, \neg \neg Q \vdash_{\mathrm{CPL}} P \wedge Q$.

2.5.1.1.3. The $\{\wedge, \vee, \neg\}$-fragment of $C P L$ as $M P L+D N E$ has the separation property This fact follows from Theorem 9 and the preceding remark.

\subsection{Generic Lindenbaum Lemma: second extension}

THEOREM 12 Where $\Delta_{0}$ and $\Delta$ are sets of formulas such that $\Sigma \cup\{A\} \subseteq \Delta_{0} \subseteq \Delta$ and $\Delta_{0}$ is closed under subformulas,

13 It is characteristic of the logic KPL that $\neg(P \wedge Q) \vdash \neg P \vee \neg Q$ is derivable in it (i.e., it is the weakest intermediate logic in which this is a theorem schema). By an argument like that used here, the formulation of KPL given in 2.2.5 does not have the subformula property. On the other hand, in the light of Theorems 5, 6 and 9, it does have the separation property. 
( $\left.v_{\mathrm{DNE}}\right)$ provided $\Delta_{0} \cup\{\neg A\} \cup\left\{\neg \neg B: B \in \Delta_{0}\right\} \subseteq \Delta$, if $\rightarrow$ occurs in $\Delta_{0}$ and the rules of $\neg-I, \rightarrow-I$, and DNE either belong to $R$ or are safely $\Delta$-derived rules given the rules in $R, \Gamma$ is $\Delta_{0}$-implication-complete.

Proof. If $B, C, B \rightarrow C \in \Delta_{0}, B \notin \Gamma$, and $B \rightarrow C \notin \Gamma$, then $\Gamma, B \vdash_{R \backslash \Delta} A$ and $\Gamma$, $B \rightarrow C \vdash_{R \backslash \Delta} A$, whence, from the former by $\neg-\mathrm{I}, \Gamma, \neg A, B \vdash_{R \uparrow \Delta} \neg \neg C$; by DNE, $\Gamma, \neg A, B \vdash_{R \backslash \Delta} C$ and so, by $\rightarrow-\mathrm{I}, \Gamma, \neg A \vdash_{R \backslash \Delta} B \rightarrow C$; from there $\Gamma, \neg A \vdash_{R \backslash \Delta} A$, as $\Gamma, B \rightarrow C \vdash_{R \backslash \Delta} A$; by $\neg-\mathrm{I}, \Gamma, \vdash_{R \backslash \Delta} \neg \neg A$ and finally, by DNE, $\Gamma \vdash_{R \uparrow \Delta} A$, which we know not to be the case.

2.5.1.1.5. A weak subformula property for CPL as MPL $+D N E$ If $\Sigma \vdash_{\mathrm{CPL}} A$ is classically valid but not derivable in MPL, then there is a CPL derivation of $A$ from premises in $\Sigma$ in which at most subformulas of formulas in $\Sigma \cup\{A\}$, the double negations of such formulas, and $\neg A$ occur.

2.5.1.2. CPL as $I P L+D N E$ We obtain a sharper restriction on subformulas in CPL derivations if we are prepared to accept redundancy in our rules governing negation. We know by GLL (iii) that $\neg$-E guarantees $\Delta$-consistency. If, in addition to DNE, we also take $\neg$-E as a basic rule of CPL, the use of double negations of members of $\Delta_{0}$ other than $A$ is circumvented: if $\Gamma, B \vdash_{R \backslash \Delta} A$ then, by $\neg$-E, $\Gamma, \neg A, B \vdash_{R \backslash \Delta} C$ directly.

2.5.1.2.1. A weak subformula property for CPL as IPL + DNE In the derivation of any sequent $\Sigma \vdash A$ that is classically valid but not derivable in IPL, only subformulas of members of $\Sigma \cup\{\neg \neg A\}$ need occur. As a corollary to Theorem 3 (Strong Glivenko Theorem) we have that DNE, which must be used in deriving the sequent, need be used only once and we may further insist that its application be the last step.

2.5.2. Classical reductio ad absurdum The proof-theoretic results of the previous subsection concerning classical negation are sensitive to the exact formulation of CPL. Instead of adding DNE to IPL, we can add the following weak rule of classical reductio ad absurdum (CRA) to IPL:

$\begin{array}{cc}\text { Classical reductio } & {[\neg A]} \\ \text { ad absurdum } & \vdots \\ & \frac{A}{A} .\end{array}$

(When we do so, $\neg$-E is not redundant.) With this formulation of CPL we obtain this result:

\subsubsection{A weak subformula property for CPL as IPL + CRA}

THEOREM 13 In the derivation of any sequent $\Sigma \vdash A$ that is classically valid but not derivable in IPL, only subformulas of members of $\Sigma \cup\{\neg A\}$ need occur. CRA, which must be used in deriving the sequent, need be used only once and we may further insist that its application be the last step.

Proof. If $\Sigma \vdash_{\mathrm{CPL}} A$, then $\Sigma \vdash_{\mathrm{CPL}} \neg \neg A$ and so, by the Strong Glivenko Theorem,

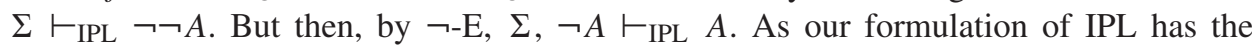
subformula property, $\Sigma, \neg A \vdash_{R I \uparrow(\Sigma \cup\{\neg A\})} A$. A final application of CRA gives us a CPL derivation meeting the stated constraints.

2.5.3. Dilemma We replace CRA with Dilemma to obtain yet a third formulation of CPL. 


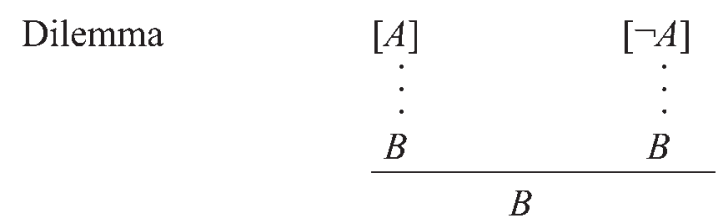

$\neg-\mathrm{I}$ and CRA in effect just are the special cases when $B=\neg A$ and $B=A$, respectively. It is as if Dilemma were designed to give us

\subsubsection{Generic Lindenbaum Lemma: third extension}

THEOREM 14 Where $\Delta$ is a set of formulas closed under subformulas and such that $\Sigma \cup\{A\} \subseteq \Delta$,

(iv $\left.v_{\mathrm{Di}}\right)$ if $\neg$ occurs in $\Delta$ and the Rule of Dilemma either belongs to $R$ or is a safely $\Delta$ derived rule given the rules in $R, \Gamma$ is $\Delta$-negation-complete.

The instances we need in order to establish negation-completeness are of the form

$$
\Gamma, B \vdash A \text { and } \Gamma, \neg B \vdash A \text { therefore } \Gamma \vdash A \text {. }
$$

2.5.3.2. The $\{\wedge, \vee, \neg\}$-fragment when $C P L=I P L+$ Dilemma $\quad$ In view of Theorem 14 and GLL (i)-(iii) we have immediately

THEOREM 15 When Dilemma is the only strictly classical rule the $\{\wedge, \vee, \neg\}$-fragment of CPL has the subformula property (and therefore the separation property).

\subsubsection{A weak subformula property for CPL as IPL + Dilemma}

THEOREM 16 In the derivation of any sequent $\Sigma \vdash$ A that is classically valid but not derivable in IPL, only subformulas of members of $\Sigma \cup\{\neg A\}$ need occur. Dilemma, which must be used in deriving the sequent, need be used only once and we may further insist that its application be the last step.

Proof. If $\Sigma \vdash_{\mathrm{CPL}} A$, then, exactly as in the proof of Theorem 13, we find that $\Sigma$, $\neg A \vdash_{R I \uparrow(\Sigma \cup\{\neg A\})} A$. As $A \vdash_{R I \Gamma(\Sigma \cup\{\neg A\})} A$, a final application of Dilemma gives us a CPL derivation satisfying the stated constraints.

\subsection{Classical implication.}

2.6.1. Peirce's Rule As is well known, we obtain CPL when we add Peirce's Rule to IPL.

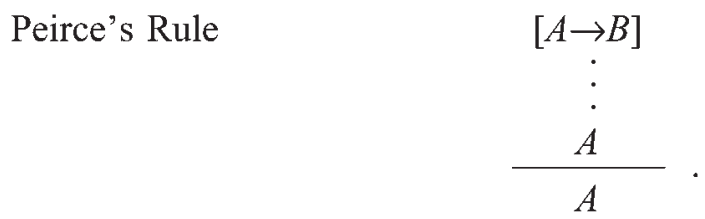

No formulation of CPL that adds to IPL solely a pure rule dealing with implication, such as Peirce's Rule, has the separation property as the classically valid, intuitionistically 
invalid $\rightarrow$-free sequent $\neg \neg P \vdash P$ is derivable in CPL. (As is well known, each of DNE and Peirce's Rule is a derived rule in any extension of IPL containing the other.) ${ }^{14}$

\subsubsection{Generic Lindenbaum Lemma: fourth extension}

THEOREM 17 Where $\Delta_{0}$ and $\Delta$ are sets of formulas such that $\Sigma \cup\{A\} \subseteq \Delta_{0} \subseteq \Delta$ and $\Delta_{0}$ is closed under subformulas,

$\left(v_{\mathrm{P}}{ }^{\prime}\right.$ rce $)$ provided that $\Delta_{0} \cup\left\{A \rightarrow C: B \rightarrow C \in \Delta_{0}\right\} \subseteq \Delta$, if $\rightarrow$ occurs in $\Delta$ and Peirce's Rule and the rules of $\rightarrow-I$ and $\rightarrow-E$ either belong to $R$ or are safely $\Delta$-derived rules given the rules in $R, \Gamma$ is $\Delta_{0}$-implication-complete.

Proof. If $B \in \Delta_{0}, C \in \Delta_{0}, B \rightarrow C \in \Delta_{0}, B \notin \Gamma$, and $B \rightarrow C \notin \Gamma$ then $\Gamma, B \vdash_{R \backslash \Delta} A$ and $\Gamma, B \rightarrow C \vdash_{R \backslash \Delta} A$. As $\Gamma, B \vdash_{R \backslash \Delta} A$, we have, by $\rightarrow-\mathrm{E}$, that $\Gamma, B, A \rightarrow C \vdash_{R \backslash \Delta} C$, whence, by $\rightarrow-\mathrm{I}, \Gamma, A \rightarrow C \vdash_{R \uparrow \Delta} B \rightarrow C$; but $\Gamma, B \rightarrow C \vdash_{R \mid \Delta} A$, so $\Gamma, A \rightarrow C \vdash_{R \mid \Delta} A$ and thus, by Peirce's Rule, $\Gamma \vdash_{R} \triangleright A$, which we know not to be the case.

2.6.1.2. A weak subformula property for the $\{\wedge, \vee, \rightarrow\}$-fragment of $C P L$ as $I P L+$ Peirce's Rule When CPL $=$ IPL + Peirce's Rule, in the derivation of any $\neg$-free sequent $\Sigma \vdash A$ that is classically valid only subformulas of members of $\Sigma \cup\{A\}$ and conditionals of the form $A \rightarrow C$ where $B \rightarrow C$ is a subformula of a member of $\Sigma \cup\{A\}$ need occur.

2.6.1.3. The $\{\wedge, \vee, \rightarrow\}$-fragment of $C P L$ as $I P L+$ Peirce's Rule has the separation property That the $\{\wedge, \vee, \rightarrow\}$-fragment of CPL, with Peirce's Rule as the only strictly classical rule, has the separation property follows from the previous result and Theorem 9.

\subsubsection{Generic Lindenbaum Lemma: fifth extension}

THEOREM 18 Where $\Delta_{0}$ and $\Delta$ are sets of formulas such that $\Sigma \cup\{A\} \subseteq \Delta_{0} \subseteq \Delta$ and $\Delta_{0}$ is closed under subformulas,

(iv ${ }_{\mathrm{P}}$ 'rce $)$ provided that $\Delta_{0} \cup\left\{A \rightarrow \neg B: \neg B \in \Delta_{0}\right\} \subseteq \Delta$, if $\neg$ occurs in $\Delta$ and Peirce's Rule and the rules of (weak) $\neg-I$ and $\rightarrow-E$ either belong to $R$ or are safely $\Delta$-derived rules given the rules in $R, \Gamma$ is $\Delta_{0}$-negation-complete.

Proof. If $B \in \Delta_{0}, \neg B \in \Delta_{0}, B \notin \Gamma$, and $\neg B \notin \Gamma$ then $\Gamma, B \vdash_{R \mid \Delta} A$ and $\Gamma, \neg B \vdash_{R \mid \Delta} A$. As $\Gamma, B \vdash_{R \backslash \Delta} A$, we have, by $\rightarrow$-E, that $\Gamma, B, A \rightarrow \neg B \vdash_{R \backslash \Delta} \neg B$ and so, by (weak) $\neg$-I, $\Gamma, A \rightarrow \neg B \vdash_{R \mid \Delta} \neg B$; as $\Gamma, \neg B \vdash_{R \mid \Delta} A$, we have that $\Gamma, A \rightarrow \neg B \vdash_{R \mid \Delta} A$, whence, by Peirce's Rule, $\Gamma \vdash_{R \mid \Delta} A$, which we know not to be the case.

\subsubsection{Weak subformula property for CPL as IPL + Peirce's Rule}

THEOREM 19 When CPL $=I P L+$ Peirce's Rule, in the derivation of any sequent $\Sigma$ $\vdash$ A that is classically valid only subformulas of members of $\Sigma \cup\{A\}$ and conditionals of the forms $A \rightarrow C$, where $B \rightarrow C$ is a subformula of a member of $\Sigma \cup\{A\}$, and $A \rightarrow \neg B$, where $\neg B$ is a subformula of a member of $\Sigma \cup\{A\}$, need occur.

14 The axiom system corresponding to the formulation of classical propositional logic that we are now considering has the separation property (in the sense appropriate to axiom systems). In addition the axioms are logically independent and dropping the axiom, Peirce's Law, corresponding to Peirce's Rule, gives an axiomatization of intuitionist propositional logic, dropping a further axiom corresponding to $\neg$-elimination gives an axiomatization of minimal propositional logic. See Kanger (1955) and Curry (1963, pp. 288, 307). 
COROLlARY 1 If at most the connectives $\neg$ and $\rightarrow$ occur in $\Sigma \cup\{A\}$ and $\Sigma \vdash_{\mathrm{CPL}} A$ then there is a derivation in which only rules governing $\neg$ and $\rightarrow$ are employed.

2.6.2. Tarski's Rule The rule that most directly allows us to establish $\Delta$-implicationcompleteness is this

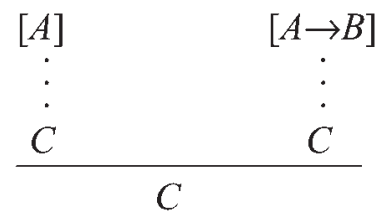

As Dilemma stands to (weak) CRA in shape, so this rule stands to Peirce's Rule. And as Peirce's Rule stands to Peirce's Law, so this rule stands to Tarski's Law. For that reason I shall call it "Tarski's Rule". ${ }^{15}$ In any logic containing the standard $\rightarrow-$ I and $\rightarrow$-E rules, Peirce's Rule and this rule are interderivable. Exactly because this rule, Tarski's Rule, gives us implication-completeness directly, we can improve upon our subformula results for CPL. Tarski's Rule is classically sound.

2.6.2.1. Strict subformula property for the $\{\wedge, \vee, \rightarrow\}$-fragment of CPL as IPL + Tarski's Rule

THEOREM 20 If $\Sigma \vdash_{\mathrm{CPL}} A$ and $\neg$ does not occur in $\Sigma \cup\{A\}$ then there is a derivation of A from premises in $\Sigma$ in which at most subformulas of formulas in $\Sigma \cup\{A\}$ occur. ${ }^{16}$

\subsubsection{Weak subformula property for CPL as IPL + Tarski's Rule}

COROLlaRY 2 If $\Sigma \vdash_{\mathrm{CPL}} A$ then there is a derivation of $A$ from premises in $\Sigma$ in which at most subformulas of formulas in $\Sigma \cup\{A\}$ and formulas of the form $A \rightarrow \neg B$, where $\neg B$ is a subformula of a formula in $\Sigma \cup\{A\}$, occur.

2.7. A formulation of CPL with the (strict) subformula property. We add to IPL both Dilemma and Tarski's Rule. As is clear in the light of 2.5.3 and 2.6.2, this gives us a formulation of CPL in which, for any classically valid sequent $\Sigma \vdash A$, there is a derivation in which only subformulas of formulas in $\Sigma \cup\{A\}$ occur.

The most obvious drawback to this most recent formulation of CPL is that there is redundancy: doubly so, for on the one hand Dilemma and Tarski's Rule are each derived rules in any extension of IPL containing the other, and, on the other, $\neg$-I is a derived rule in any logic containing Dilemma and $\neg$-E. These two sources of redundancy are not quite on a par. In obtaining $\neg$-I from Dilemma and $\neg$-E, we need introduce no formulas not in the original application of $\neg$-I:

15 For the name 'Tarski's Law', see Thomas (1960). What Thomas calls 'Tarski's Law with antecedents commuted' occurs in the statement of a result due to Tarski in Łukasiewicz \& Tarski (1930, sec. 4). Prior (1962, p. 302), credits a version of the uncommuted form with propositional quantifiers to an unnamed 1921 publication of Tarski's. On p. 319 he calls the axiom schema 'Tarski or Dilemma', explaining the latter by a substitution of, first, a falsum constant for ' $q$ ' then a negation $\mathrm{Np}$ for $\mathrm{Cp} 0$ to obtain the familiar form of Dilemma. Prior's index features the entry 'Law of' under 'Tarski'.

16 Given the strict subformula property, the $\{\wedge, \vee, \rightarrow\}$-fragment of CPL as IPL + Tarski's Rule $=$ the $\{\wedge, \vee, \rightarrow\}$-fragment of MPL + Tarski's Rule. We have, then, that if $C(\Sigma \cup A) \subseteq\{\wedge, \vee, \rightarrow\}$ and $\Sigma \vdash_{\text {CPL }} A$ then $\Sigma \vdash_{\text {MPL }+ \text { Tarski }} A$. Compare Parsons (1966, p. 254, theorem 1). 

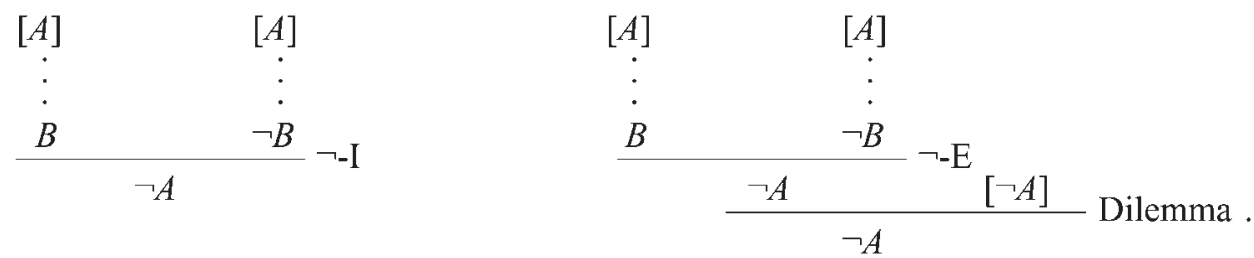

The remedy is straightforward: drop $\neg-\mathrm{I}$. But, just for a moment, we'll hold off doing that.

We can obtain the effect of Dilemma from Tarski's Rule, $\neg-\mathrm{I}$, and $\rightarrow$-E but we may have to introduce the conditional $A \rightarrow \neg A$, where $A$ and $\neg A$ are the assumptions discharged in the application of Dilemma, to do so; we can obtain the effect of Tarski's Rule from Dilemma, $\neg$-E, and $\rightarrow$-I but we may have to introduce the negated formula $\neg A$, where $A$ and $A \rightarrow B$ are the assumptions discharged in the application of Tarski's Rule, to do so. (There's a fairly obvious symmetry here.)
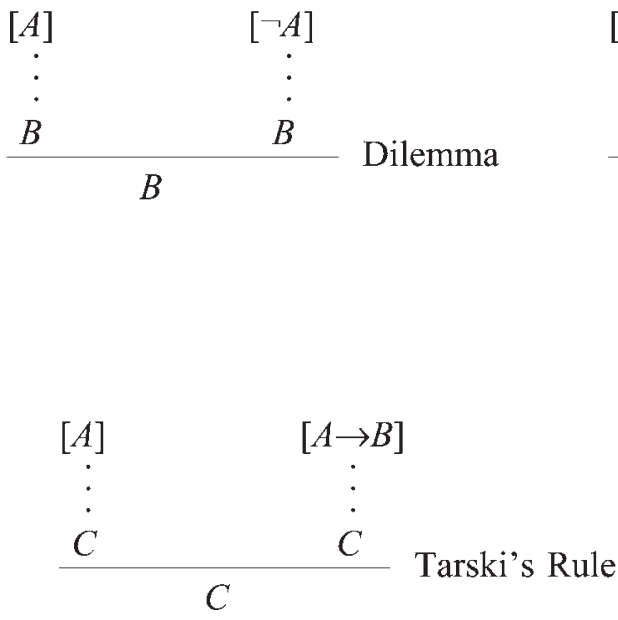
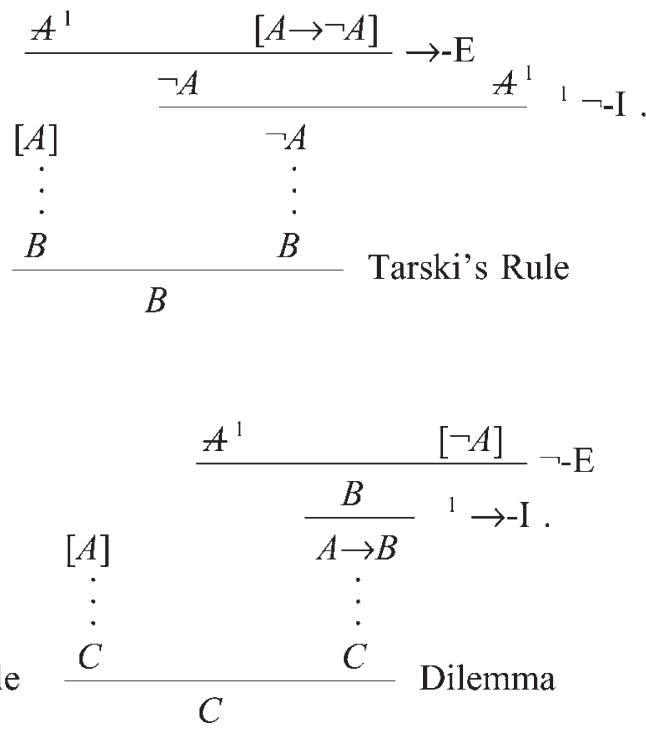

There are, of course, other ways to obtain the effect of each of Tarski's Rule and Dilemma by employing the other. There is none, however, that succeeds in doing so without, in some cases, introducing formulas that do not occur as subformulas in the original application. For suppose that one could, say, obtain the effect of Tarski's Rule from Dilemma without introducing new formulas; then we could simply drop Tarski's Rule from our formulation of CPL while still respecting the subformula property for derivations in CPL, but we know, by the theoremhood of $((P \rightarrow Q) \rightarrow P) \rightarrow P$, that this is impossible. Likewise, the derivability of $\neg \neg P \vdash P$ shows the same for attempts to obtain Dilemma from Tarski's Rule. Tarski's Rule and Dilemma are pure rules. By an argument parallel to that just given we see that

THEOREM 21 There is no formulation of CPL as an extension of IPL employing pure rules such that: $(i) \neg$ and $\rightarrow$ are both primitive; (ii) the rules governing connectives are pure rules; (iii) none of the rules is redundant, that is, derivable given the others; (iv) 
for any derivable sequent $\Sigma \vdash_{\mathrm{CPL}} A$, there is a derivation in which only subformulas of members of $\Sigma \cup\{A\}$ occur.

2.7.1. Excising the Tarski's Rule/Dilemma redundancy We can rid ourselves of the Tarski's Rule/Dilemma redundancy by treating negation not as primitive but as defined. Let $\perp$ be a 0 -ary connective with the single elimination rule

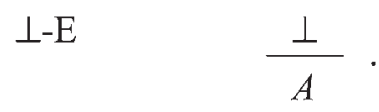

$\neg A$ abbreviates $A \rightarrow \perp$. Dilemma is now revealed as merely a special case of Tarski's Rule, just as $\neg-\mathrm{I}$, which we know to be dispensable, is a combination of $\rightarrow$-E followed by $\rightarrow$-I, and $\neg$-E comprises $\rightarrow$-E followed by $\perp$-E. Theorem 20 takes care of the rest. This is, perhaps, the orthodox modern approach.

What we might call the "old time classical approach", an approach presumably motivated from a strictly classical point of view only, treats $A \rightarrow B$ as an abbreviation of $\neg A \vee B . \rightarrow-\mathrm{I}$ is obtained as $\vee$-I followed by Dilemma; $\rightarrow$-E is $\neg$-E followed by $\vee$-E; and $\checkmark$-I and Dilemma give us Tarski's Rule. Theorem 15 takes care of the rest.

We have noted that $\neg$-I is redundant. If we drop it we block the derivation above of Dilemma from Tarski's Rule-and, as we'll see shortly, block any derivation of it. On the other hand, we'll still be able to derive Tarski's Rule from Dilemma, $\neg$-E, and $\rightarrow$-I. Now, as careful attention to our completeness theorems will show, in the presence of Tarski's Rule we make a single type of use of $\rightarrow-I$ in the completeness theorem: we infer a conditional directly from its consequent. That's it. We no longer make use of the full strength of the rule $\rightarrow-I$ in the completeness theorem. This suggests that we may restrict $\rightarrow-I$. We can. Given Tarski's Rule and this weak form of $\rightarrow-$ I

$$
\text { weak } \rightarrow \text {-I } \frac{B}{A \rightarrow B}
$$

we can derive the standard introduction rule for implication. Notice that in obtaining the standard $\rightarrow$-I from Tarski's Rule and weak $\rightarrow$-I we need introduce no formulas not in the original application of $\rightarrow-\mathrm{I}$ :

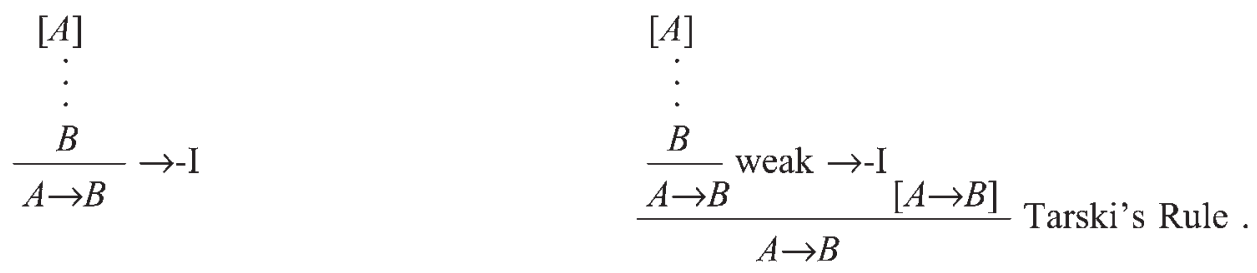

Our chosen formulation of CPL, then, has the rules $\wedge$-I, $\wedge-E, \vee-I, \vee-E$, weak $\rightarrow-I$, Tarski's Rule, $\rightarrow$-E, Dilemma, and $\neg$-E. The rules are independent. We need check this only in the case of negation and implication. Giving the other connectives their classical interpretations, reading negations as uniformly true makes Dilemma sound but not $\neg$-E, reading negations as uniformly false makes $\neg$-E sound but not Dilemma. Giving the other connectives their classical interpretations, reading conditionals as uniformly true makes weak $\rightarrow-$ I and Tarski's Rule sound but not $\rightarrow-E$; reading $A \rightarrow B$ as $B$ (i.e., as true if and only if its consequent is true) makes weak $\rightarrow-$ I and $\rightarrow-$ E sound but not Tarski's Rule (as 
$A \vee B$ is not a classical theorem schema); reading $A \rightarrow B$ as $\neg A$ (i.e., as true if and only if its antecedent is false) makes Tarski's Rule and $\rightarrow$-E sound but not weak $\rightarrow-$ I.

2.7.2. Further proof-theoretic consideration regarding this formulation of CPL I shall treat Dilemma and Tarski's Rule as introduction rules for, respectively, negation and implication: each allows the introduction of formulas with the respective connective dominant as dischargeable assumptions. (Weak $\rightarrow-$ I and Tarski's Rule are both introduction rules for implication.) Doubtless this strikes the reader as odd. Neither satisfies the general form for introduction rules proposed by Prawitz, ${ }^{17}$ and Tarski's Rule is a generalization of Peirce's Rule, a variation on which has been, at Kanger's suggestion, used by Hugues Leblanc as the sole elimination rule for the conditional. ${ }^{18}$ On the other hand, introduction rules satisfying Prawitz's general form can be rewritten to appear, like Dilemma and Tarski's Rule, as rules for the discharging of assumptions. The idea is this: the orthodox formulation of an introduction rule presents certain grounds as sufficient for inferring a formula with some connective dominant; if, instead of inferring that formula, one has already used it as an assumption, then these grounds suffice to show that the assumption is unnecessary, it can be discharged, for one has to hand all that is needed to make do without it. Turning this on its head, I suggest that we can take introduction rules to be rules licensing the discharge of assumptions with connectives dominant. For example, in place of the orthodox introduction rule for $\wedge$, we would have

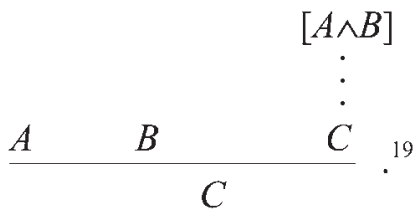

Similarly, the orthodox rule of $\rightarrow$-introduction, with which we are, of course, dispensing, would take this form:

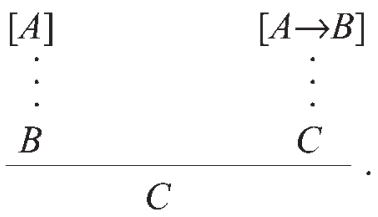

The standard rules are immediately recoverable from these, so we shall retain the standard introduction rules for conjunction and disjunction.

The discharge-as-assumption format for introduction rules is more general than the orthodox one. It allows us to count Dilemma and Tarski's Rule as introduction rules. I am not here going to defend the claim that we should so count them. ${ }^{20}$

17 See Prawitz (1979, p. 35).

18 The classically valid $(P \rightarrow Q) \rightarrow P, P \rightarrow R \vdash R$ underpins Leblanc's elimination rule. See, for example, Beth \& Leblanc (1960) and Leblanc (1982, pp. 285, 291).

19 Compare the style of rule used in Michel Parigot's "free deduction" in, for example, Parigot (1993). I owe this reference to James McKinna.

20 As we retain the standard elimination rules $-\neg-\mathrm{E}$ and $\rightarrow-\mathrm{E}-$ and the standard introduction rules for negation and the conditional are derived rules, these new introduction rules are in harmony with the elimination rules in the sense of Tennant's (1978, pp. 74-75) Natural Logic. 
As Stephen Read pointed out to me, we can put weak $\rightarrow-\mathrm{I}$ in this dischargeablecomplex-assumption form:

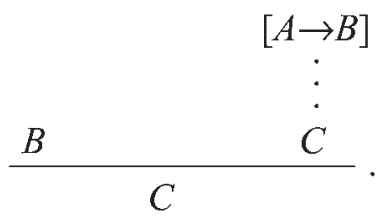

In what follows we may let 'weak $\rightarrow-I$ ' refer indifferently to either form of the rule.

\subsection{The extension of MPL variously known as $L D, J X$, and $M C^{+} .21$}

This logic, which I shall call DPL, can be formulated by adding Dilemma to MPL. In what follows, $R D$ denotes the set of rules obtained by adding Dilemma to the formulation of MPL above.

As formulated here, DPL shares with our earlier formulation of IPL the following three properties: (i) $\neg$ and $\rightarrow$ are both primitive; (ii) the rules governing connectives are pure rules; (iii) none of the rules is redundant. Only (iii) requires argument. To secure the conclusion we need only show that $\neg$-inv and Dilemma are independent of each other. First, let $\neg A$ be read as an abbreviation for $A \rightarrow P$, for some atomic formula $P$ : then Dilemma, which turns out to be special case of Tarski's Rule, is intuitionistically invalid but $\neg$-inv is intuitionistically acceptable. Second, let $\phi$ associate with each formula of $\mathscr{L}$ a formula of $\mathscr{L}$ and let $\neg A$ abbreviate $A \rightarrow \phi(A)$ : then Dilemma, being a case of Tarski's Rule is classically valid, but by appropriate choices of $A, B$, and $\phi$ it is easy to arrange for an instance of $\neg$-inv to be classically invalid.

2.8.1. Kripke semantics for DPL DPL is known to be sound and complete with respect to Kripke models $<T, \preceq, u, \mathscr{F}>$ for MPL satisfying the additional constraint:

$$
\forall s, t[\text { if } t \prec s \text { then } s \in \mathscr{F}] \text {; }
$$

"no node is more than one step away from $\mathscr{F} " .22$

\subsubsection{Strong Glivenko Theorem for DPL}

THEOREM 22 If $\Sigma \vdash_{\text {DPL }} \neg A$ then $\Sigma \vdash_{\text {MPL }} \neg A .^{23}$

Proof. Let $\Delta$ contain all and only subformulas of the members of $\Sigma \cup\{\neg A\}$. $\neg$-I is one of the rules of MPL, so if $\Sigma \nvdash_{\text {MPL }} \neg A$ then $\Sigma \nvdash_{R M \backslash \Delta} \neg A$ and there is, by GLL (i)-(iv), a $\Delta$ prime, $\Delta$-consistent, $\Delta$-negation-complete, $\Delta, R M \uparrow \Delta$-theory $\Gamma$, a subset of $\Delta$, extending $\Sigma$ and not containing $\neg A$ and such that for $B \in \Delta, B \notin \Gamma$ iff $\Gamma, B \vdash_{R M \uparrow \Delta} \neg A$. As $\neg A \notin$ $\Gamma$ and $\Gamma$ is $\Delta$-negation-complete, $A \in \Gamma$ and hence every $\Delta$-prime $\Delta, R M \uparrow \Delta$-theory $\Theta$

21 LD is Curry's name for its sequent calculus formulation; he calls its natural deduction formulation, for which he adds our (weak) CRA to MPL, TD (Curry, 1963, pp. 260, 280). $\mathrm{MC}^{+}$ and JX are from Parsons (1966), and Segerberg (1968), respectively.

Curry calls LD the system of complete refutability or strict negation, prompted, he says, by a remark of Johansson's (1937, p. 260, n. 2, cf. pp. 286, 306) that 'it might be suitable as a theory of strict implication'. Johansson (1937, p. 129) was rather more circumspect than Curry's footnote suggests, saying only that 'some similarity with Lewis's calculus of strict implication is to be expected but a complete agreement is out of the question [ausgeschlossen]'.

22 See Segerberg (1968, p. 38).

23 Compare Curry (1963, p. 279). 
contained in $\Delta$ and properly extending $\Gamma$ is $\Delta$-inconsistent. As $\mathscr{F}$ contains exactly the $\Delta$ inconsistent $\Delta, R M \backslash \Delta$-theories in $\Delta$ that extend $\Gamma$, the model satisfies the constraint that $\Theta \in \mathscr{F}$ when $\Gamma \subsetneq \Theta$, and hence is a model for DPL.

\subsubsection{The $\{\wedge, \vee, \rightarrow\}$-fragment of $D P L$}

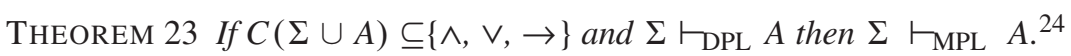

Proof. Suppose that $\Sigma \nvdash_{\text {MPL }} A$. Let $\Delta$ be the least subset of $\mathscr{L}$ extending $\Sigma \cup\{A\}$ and closed under subformulas. By the proof of Theorem 7, the generic completeness theorem for MPL, there is a Kripke model whose nodes, ordered by inclusion, are those subsets of $\Delta$ that constitute $\Delta$-prime $\Delta, R M \uparrow \Delta$-theories extending the base node $\Gamma ; \Sigma \subseteq \Gamma$ and $A \notin \Gamma$. As $\Sigma \cup\{A\}$ is $\neg$-free, $\mathscr{F}$, the set of $\Delta$-inconsistent, $\Delta$-prime, $\Delta, R M \backslash \Delta$-theories in $\Delta$ extending $\Gamma$, is empty. (Our model is thereby a Kripke model for IPL, showing that $\Sigma \nvdash_{\text {IPL }}$ A.) The set $\mathscr{F}$ in a Kripke model for MPL plays no role in the evaluation of $\neg$-free formulas. We can therefore modify the model the generic completeness theorem delivers by reassigning $\mathscr{F}$ as we please: rather than be the empty set, we take it to include all nodes. We now have a Kripke model for DPL at the base node of which all members of $\Sigma$ are verified and $\mathrm{A}$ is not. By the soundness of DPL, $\Sigma \nvdash_{\mathrm{DPL}} A$.

2.8.4. Weak subformula property for DPL We aim to show that when $\Sigma \vdash_{\text {DPL }} A$ there is a derivation of $A$ from premises in $\Sigma$ in which at most subformulas of formulas in $\Sigma \cup\{\neg A\}$ occur. Exactly because $\neg$-E is not a (derived) rule of DPL, we cannot emulate the quick line of argument used in Theorem 16 for CPL as IPL + Dilemma. Consequently, we have to sketch a proof of completeness. As the proof closely mimics the completeness theorem for MPL in Theorem 7 we can be brief.

\subsubsection{Generic completeness theorem}

THEOREM 24 If $\Sigma \nvdash_{R D \uparrow(\Sigma \cup\{\neg A\})}$ A then there is a Kripke model $<T, \preceq, u, \mathscr{F}>$ with a base node such that all members of $\Sigma$ are verified at the base node but $A$ is not verified there and all members of $T$ other than perhaps the base node belong to $\mathscr{F}$.

Proof. Let $\Delta$ be the least subset of $\mathscr{L}$ extending $\Sigma \cup\{\neg A\}$ and closed under subformulas. By GLL, (i) and (ii), there is a $\Delta$-prime, not necessarily $\Delta$-consistent, $\Delta, R D \backslash \Delta$-theory $\Gamma$ contained in $\Delta$, extending $\Sigma$ and not containing $A$. For all $B \in \Delta, B \notin \Gamma$ iff $\Gamma$, $B \vdash_{R D 「(\Sigma \cup\{\neg A\})} A$. By Theorem 14, $\Gamma$ is $\Delta$-negation-complete as $\neg A \in \Delta$. The nodes of our Kripke model are those subsets of $\Delta$ that constitute $\Delta$-prime, $\Delta$-negation-complete $\Delta$, $R D\lceil\Delta$-theories extending $\Gamma$. $\mathscr{F}$ is the set of $\Delta$-inconsistent, $\Delta$-prime, $\Delta, R D \backslash \Delta$-theories in $\Delta$ extending $\Gamma$. As ever, $\Gamma$ is the base node, $\preceq$ is the subset relation, and we stipulate that the atomic formula $B$ is verified at the $\Delta, R D \backslash \Delta$-theory-node $\Theta$ iff $B \in \Theta . \neg A \in \Gamma$ by $\Delta$-negation-completeness, and $A$ belongs to any $\Delta$-prime, $\Delta$-negation-complete $\Delta, R D \backslash \Delta$ theory properly extending $\Gamma$, hence every such theory is a member of $\mathscr{F}$.

COROLLARY 3 If $\Sigma \vdash_{\text {DPL }} A$ then there is a derivation of A from premises in $\Sigma$ in which at most subformulas of formulas in $\Sigma \cup\{\neg A\}$ occur.

\subsubsection{DPL has the separation property}

COROLlary 4 This is an immediate consequence of the last result, Theorem 23, and MPL's possession of the (subformula hence) separation property.

24 Parsons (1966, p. 257, Theorem 3). Parsons offers a rather different proof. 
2.8.5. DPL as MPL + Dilemma does not have the subformula property

FACT $1 \quad(P \rightarrow-Q) \rightarrow P \vdash_{\mathrm{DPL}} P$.

$$
\frac{\frac{\frac{P^{1} \quad-P^{2}}{\neg Q}{ }^{-\mathrm{I}}}{P \rightarrow-Q}{ }^{1} \rightarrow-\mathrm{I}(P \rightarrow \neg Q) \rightarrow P}{\frac{P}{P} \rightarrow-\mathrm{E}} \frac{P^{2}}{P}{ }^{2} \text { Dilemma }
$$

FACT $2 \neg Q,(P \rightarrow \neg Q) \rightarrow P \vdash_{\mathrm{MPL}} P$.

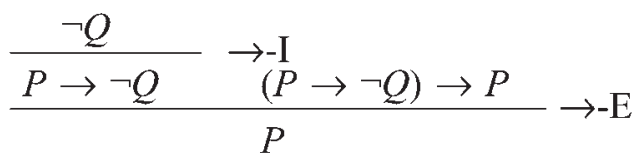

FACT $3 Q,(P \rightarrow \neg Q) \rightarrow P \nvdash_{\mathrm{IPL}} P$. See Figure 5 .

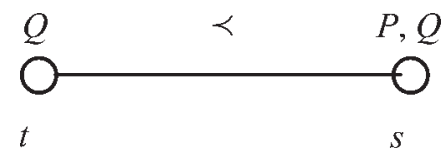

Fig. 5.

$v_{s}(P \rightarrow \neg Q)=v_{t}(P \rightarrow \neg Q)=0$, hence $v_{t}((P \rightarrow \neg Q) \rightarrow P)=1 ; v_{t}(Q)=1$; but $v_{t}(P)=0$.

THEOREM 25 DPL as MPL + Dilemma does not have the subformula property.

Proof. In view of Facts 1 and 3, if DPL were to have the subformula property there would be a DPL proof of $P$ from $(P \rightarrow \neg Q) \rightarrow P$ in which, of necessity, Dilemma is used and in any such use the assumptions discharged are $Q$ and $\neg Q$. Consider such a putative derivation. This being propositional logic, the last use of Dilemma can be deferred so as to be the last step in the derivation (cf. Tennant, 1978, p. 94). Thus the (rearranged) proof contains two subproofs, one with undischarged assumptions belonging to the set $\{Q$, $(P \rightarrow \neg Q) \rightarrow P\}$, the other to $\{\neg Q,(P \rightarrow \neg Q) \rightarrow P\}$. In view of Fact 2 , the subproof in which $\neg Q$ occurs as undischarged assumption either is or can be replaced without loss by a Dilemma-free MPL derivation of $P$ from $\neg Q$ and $(P \rightarrow \neg Q) \rightarrow P$. Should there be an application of Dilemma in the subproof with $Q$ as undischarged assumption, there is a last such and we can carry out the same procedure, deferring its application until the penultimate step of the proof; this subproof must itself contain a subproof in which $\neg Q$ occurs as undischarged assumption and, again, this subproof either is or can be replaced without loss by a Dilemma-free MPL derivation of $P$ from $\neg Q$ and $(P \rightarrow \neg Q) \rightarrow P$. (This in fact renders the first application of Dilemma considered redundant, so it can be deleted without loss.) Repeating as many times as need be, what we end up with is a proof in which there is an application of Dilemma above which stands a Dilemma-free MPL 
derivation of $P$ from $Q$ and $(P \rightarrow \neg Q) \rightarrow P$, but Fact 3 assures us that there is none such.

\section{§3. First-order logics}

3.1. The basic Lindenbaum-Henkin construction. We suppose given a first-order language $\mathscr{L}$ with a countable infinity of variables, countable, possibly empty collections of constants and unary, binary, tertiary, ..., predicates, the connectives $\wedge, \vee, \rightarrow$, and $\neg$, and the quantifier-forming prefixes $\forall$ and $\exists .{ }^{25}$ Rules of formation for formulas are standard. As is common in systems of natural deduction we do not countenance free variables. Instead, we have at our disposal a countable infinity of parameters (constants) distinct from all formulas and components of formulas in $\mathscr{L}$. We expand the stock of formulas by permitting parameters to take the place of constants. Such formulas, containing parameters, may occur in derivations. (Parameters enter and exit in the course of derivations. Typically they are not interpreted in furnishing models of formulas in the language.)

As is standard, where $v$ is a variable, an instance of a formula headed by the quantifier $\forall v$ or $\exists v$ is obtained by dropping the quantifier and uniformly substituting a name or parameter for all occurrences of the variable bound by that quantifier; a parametric instance is obtained when a parameter is used to make the substitution. Given formulas $\forall v A$ or $\exists v A$ and the constant or parameter $c$, we write $A(v / c)$ for the formula that results when the quantifier is dropped and $c$ is uniformly substituted for all occurrences of $v$ bound by whichever of $\forall v$ or $\exists v$ heads the formula; $A(v / c)$ is a subformula of both $\forall v A$ and $\exists v A$. We call a formula headed by a universal quantifier a universal generalization; likewise, an existential generalization is headed by an existential quantifier.

Given any of the logics of Part I, its first-order correlate is obtained by adding the standard introduction and elimination rules for the universal and existential quantifiers. We obtain an abbreviation for it by replacing ' $P$ ' by ' $Q$ ' in the abbreviation for the propositional logic, for example, $I Q L$ is intuitionist first-order logic.

Given a set $\Delta$ of formulas of a language $\mathscr{L}$ and a set $R$ of rules of inference, to the definitions at the beginning of 2.1 we add:

(vi) a set of formulas $\Gamma$ is $\Delta$-witness-complete if

for all existential generalizations $\exists v A$ in $\Delta, \exists v A \in \Gamma$ only if $A(v / c) \in \Gamma$ for some constant $c$ in $\mathscr{L}$.

\subsubsection{The Generic Lindenbaum-Henkin Lemma (GLHL)}

THEOREM 1 Given $R$, when $\Sigma \nvdash_{R} A$ and where $c_{0}, c_{1}, \ldots, c_{n}, \ldots$, is an infinite sequence of constants all foreign to $\Sigma \cup\{A\}$ let $\Delta_{0}$ contain all subformulas of members of $\Sigma \cup\{A\}$ formed using $c_{0}, c_{1}, \ldots, c_{n}, \ldots$ and whatever other constants may occur in members of $\Sigma \cup\{A\}$. Let $\Delta_{0} \subseteq \Delta$, and let $R \backslash \Delta$ stand for the restriction of the rules in $R$ obtained by excluding rules governing those connectives and quantifiers that do not occur in any member of $\Delta$ and restricting those that remain to formulas in $\Delta$ and parametric instances of these, that is, only formulas in $\Delta$ and instances of them obtained by instantiating with parameters alien to $\Delta$, may occur as assumptions, major and minor premises, and/or conclusions in applications of these rules, hence only formulas in $\Delta$

25 A cursory glance through a number of texts shows that there's no agreement in usage: some authors call $\forall$ and $\exists$ quantifiers, some, as I shall do here, reserve that title for the combinations $\forall \mathrm{x}, \forall \mathrm{y}, \exists \mathrm{x}, \exists \mathrm{y}$, and their like, and yet others make no clear commitment between these alternatives. 
and parametric instances of these may occur in proofs constructed using the rules in $R \uparrow \Delta .{ }^{26}$ Then

(i) there is a $\Delta_{0}, R \backslash \Delta$-theory $\Gamma$ such that $\Sigma \subseteq \Gamma \subseteq \Delta_{0}, A \notin \Gamma$, and

(a) for all formulas $B$ in $\Delta_{0}, B \notin \Gamma$ if, and only if, $\Gamma, B \vdash_{R \backslash \Delta} A$,

(b) if $\wedge$ occurs in $\Delta_{0}$ and the rules of $\wedge-I$ and $\wedge-E$ either belong to $R$ or are safely $\Delta$-derived rules given the rules in $R$, then for all formulas $B, C i n \Delta_{0}$, if $B \wedge C \in \Delta_{0}$ then $B \wedge C \in \Gamma$ if, and only if, $B \in \Gamma$ and $C \in \Gamma$,

(c) if $\rightarrow$ occurs in $\Delta_{0}$ and the rule of $\rightarrow-I$ either belongs to $R$ or is a safely $\Delta$ derived rule given the rules in $R$, then for all formulas $B, C$ in $\Delta_{0}$, if $B \rightarrow$ $C \in \Delta_{0}$ then $B \rightarrow C \in \Gamma$ if $C \in \Gamma$,

(d) if $\rightarrow$ occurs in $\Delta_{0}$ and the rule of $\rightarrow-E$ either belongs to $R$ or is a safely $\Delta$-derived rule given the rules in $R$, then for all formulas $B, C$ in $\Delta_{0}$, if $B \rightarrow C \in \Delta_{0}$ then $B \rightarrow C \in \Gamma$ only if $B \notin \Gamma$ or $C \in \Gamma$,

(e) if a universal generalization belongs to $\Delta_{0}$ and the rule of $\forall$-E either belongs to $R$ or is a safely $\Delta$-derived rule given the rules in $R$, then any universal generalization in $\Delta_{0}$ belongs to $\Gamma$ only if all its instances employing constants in $\Delta_{0}$ belong to $\Gamma$ too,

(f) if an existential generalization belongs to $\Delta_{0}$ and the rule of $\exists$-I either belongs to $R$ or is a safely $\Delta$-derived rule given the rules in $R$, then an existential generalization in $\Delta_{0}$ belongs to $\Gamma$ when any of its instances belonging to $\Delta_{0}$ belongs to $\Gamma$;

(ii) if $\vee$ occurs in $\Delta_{0}$ and the rules of $\vee-I$ and $\vee-E$ either belong to $R$ or are safely $\Delta$-derived rules given the rules in $R, \Gamma$ is $\Delta_{0}$-prime;

(iii) if $\neg$ occurs in $\Delta_{0}$ and the rule of $\neg$-E either belongs to $R$ or is a safely $\Delta$-derived rule given the rules in $R, \Gamma$ is $\Delta_{0}$-consistent;

(iv $v_{1}$ ) if $A=\neg D$, for some $D \in \Delta_{0}$ and the rule $\neg$-inv either belongs to $R$ or is a safely $\Delta$-derived rule given the rules in $R, \Gamma$ is $\Delta_{0}$-consistent;

(iv $)$ if $A=\neg D$, for some $D \in \Delta_{0}$ and the rule of $\neg$-I either belongs to $R$ or is a safely $\Delta$-derived rule given the rules in $R, \Gamma$ is $\Delta_{0}$-consistent and $\Delta_{0}$-negationcomplete;

(v) if $A=\neg D$, for some $D \in \Delta_{0}, \rightarrow$ occurs in $\Delta_{0}$ and the rules of weak $\neg-I, \neg-E$, and $\rightarrow-I$ either belong to $R$ or are safely $\Delta$-derived rules given the rules in $R, \Gamma$ is $\Delta_{0}$-implication-complete;

(vi) if an existential generalization belongs to $\Delta_{0}$ and the rule of $\exists-E$ belongs to $R$ or is a safely $\Delta$-derived rule given the rules in $R$ then $\Gamma$ is $\Delta_{0}$-witnesscomplete.

Proof. Here again we mimic the standard proof.

Let $B_{0}, B_{1}, \ldots, B_{n}, \ldots$ be an enumeration of the formulas in $\Delta_{0}$, an enumeration indexed by $\mathbb{N}$. We define a sequence of extensions of $\Sigma$ as follows:

26 Given that proofs are finite we could always use constants belonging to $\Delta$ but as we allow infinite sets of premises (all but finitely many unused in any given derivation) we would keep having to speak of derivations from subsets. It's just neater to allow parametric instances using constants alien to $\Delta$. 
(A) $\Gamma_{0}=\Sigma$;

for all $n \in \mathbb{N}$,

(B) $\Gamma_{n+1}=\Gamma_{n}$ if $\Gamma_{n}, B_{n} \vdash_{R \mid \Delta} A$,

$\Gamma_{n+1}=\Gamma_{n} \cup\left\{B_{n}\right\}$ if $\Gamma_{n}, B_{n} \nvdash_{R \mid \Delta} A$ and $B_{n}$ is not an existential generalization,

$\Gamma_{n+1}=\Gamma_{n} \cup\{\exists v D, D(v / c)\}$, where $D(v / c)$ is an instance employing a constant not occurring in $\Gamma_{n} \cup\left\{B_{n}, A\right\}$, if $B_{n}$ is the formula $\exists v D$ and $\Gamma_{n}, B_{n} \nvdash_{R \mid \Delta} A$.

We set $\Gamma=\bigcup_{n \in \mathbb{N}} \Gamma_{n}$.

Supplementary to the proof of Theorem 2.1, it does not follow by definition of the sequence $\left(\Gamma_{n}\right)_{n \in \mathbb{N}}$ that $\Gamma_{n+1} \nvdash_{R \backslash \Delta} A$ for when $B_{n}$ is an existential generalization and $\Gamma_{n}$, $B_{n} \nvdash_{R \backslash \Delta} A$ we add two formulas to $\Gamma_{n}$ to obtain $\Gamma_{n+1}$, not just $B_{n}$ itself. Suppose, then, that $B_{n}=\exists v D$ and that $\Gamma_{n}, \exists v D, D(v / c) \vdash_{R \mid \Delta} A$. As $c$ is, by design, foreign to $\Gamma_{n} \cup\{\exists v D, A\}$, its occurrence in an $R \backslash \Delta$-proof of $A$ from $\Gamma_{n} \cup\{\exists v D, D(v / c)\}$ is essentially parametric, and so, as $\exists$-E belongs to $R \backslash \Delta$ (and without overstepping the bounds of what counts as an $R\left\lceil\Delta\right.$-proof), we have that $\Gamma_{n}, \exists v D \vdash_{R \backslash \Delta} A$, which, by hypothesis, is not the case. This, and the theoryhood of $\Gamma$, ensures that $\Gamma$ is $\Delta_{0}$-witness-complete. (i) $(e)$ and $(f)$ are immediate in the light of the $\Delta_{0}, R \backslash \Delta$-theoryhood of $\Gamma$.

To save on repetition below, we shall call a $\Delta_{0}, R \backslash \Delta$-theory well behaved when it satisfies Clauses (i) $(b)-(f)$ in the statement of GLHL.

3.1.2. Strong Glivenko Theorems for fragments of classical first-order logic (CQL) By the GLHL we obtain a set $\Gamma$ that contains an existentially quantified formula just in case it contains an instance of that formula. Bearing that fact in mind and independently of any particular formulations of classical first-order logic as an extension of minimal and intuitionist first-order logics we have, as immediate consequences of GLHL, the following, where $R M Q$ denotes the set of rules in our chosen formulation of MQL, and, likewise, $R I Q$ the set of rules in our chosen formulation of IQL. Putting our earlier notation to a related use, given a set of formulas $\Delta$ we write $C(\Delta)$ for the set of connectives and quantifier prefixes that occur in at least one member of $\Delta$.

\subsubsection{Strong Glivenko Theorem for the $\{\wedge, \vee, \neg, \exists\}$-fragment of $C Q L$}

THEOREM 2 If $C(\Sigma \cup\{\neg A\}) \subseteq\{\wedge, \vee, \neg, \exists\}$ and $\Sigma \vdash_{\mathrm{CQL}} \neg A$ then $\Sigma \vdash_{\mathrm{MQL}} \neg A$.

Proof. Let $\Delta$ contain all subformulas of members of $\Sigma \cup\{\neg A\}$ formed using $c_{0}, c_{1}, \ldots, c_{n}, \ldots$ and whatever other constants may occur in members of $\Sigma \cup\{\neg A\}$. Adding to the proof of Theorem 2.2, if $\Sigma \nvdash_{\mathrm{MQL}} \neg A$, there is, by GLHL (i)-(iv) and (vi), a $\Delta$-prime, $\Delta$-consistent, $\Delta$-negation-complete, vacuously $\Delta$-implication-complete, $\Delta$-witness-complete, $\Delta, R M Q\lceil\Delta$-theory $\Gamma$, a subset of $\Delta$, extending $\Sigma$ and not containing $\neg A$. This determines a classical assignment of truth-values under which all members of $\Sigma$ are assigned the value true and $\neg A$ is assigned the value false. By the soundness of CQL with respect to (classical) truth-value semantics, $\Sigma \nvdash_{\mathrm{CQL}} \neg A$.

3.1.2.2. Strong Glivenko Theorem for the $\{\wedge, \vee, \neg, \rightarrow \exists\}$-fragment (the $\forall$-free fragment) of $C Q L$

THEOREM 3 If $C(\Sigma \cup\{\neg A\}) \subseteq\{\wedge, \vee, \neg, \rightarrow, \exists\}$ and $\Sigma \vdash_{\mathrm{CQL}} \neg A$ then $\Sigma \vdash_{\mathrm{QL}} \neg A{ }^{27}$

Proof. Building on the proofs of Theorems 2 and 2.3, GLHL (v) ensures $\Delta$-implicationcompleteness of $\Gamma$.

27 This is a strengthening of Fitting (1969, Theorem 8.3, p. 52). 


\subsection{Intuitionist first-order logic (IQL).}

3.2.1. Kripke semantics for intuitionist first-order logic A Kripke model is a quadruple $<T, \preceq, D, u>$ where

(a) $T$ is a nonempty set (of nodes);

(b) $\preceq \subseteq T^{2}$ and partially orders $T$;

(c) the function $D$ associates with each node $t$ a set $D_{t}$ in such a way that if $s \preceq t$ then $D_{s} \subseteq D_{t}$ and $\cap_{t \in T} D_{t}$ is nonempty;

(d) the function $u$ associates with each constant an element of $\cap_{t \in T} D_{t}$ and with each node $t$ and $n$-ary predicate $\phi$ a subset of $D_{t}^{n}$ in such a way that if $s \preceq t$ then $u_{s}(\phi) \subseteq$ $u_{t}(\phi)$

(e) we expand the language $\mathscr{L}$ by adding a name for each element of $\cup_{t \in T} D_{t}$; we take $\bar{d}$ to be the name of $d$, so that $u(\bar{d})=d$; we call the expanded language $\mathscr{L}_{D}$.

The function $v: T \times \mathscr{L}_{D} \rightarrow\{0,1\}$ builds on $u$ in the following ways:

(f) where $c_{1}, c_{2}, \ldots, c_{n}$ are not necessarily distinct constants of $\mathscr{L}_{D}$ and $\phi$ an $n$-ary predicate in $\mathscr{L}$, for the atomic formula $\phi c_{1} c_{2} \ldots c_{n}$ we have that $v_{t}\left(\phi c_{1} c_{2} \ldots c_{n}\right)=1$ iff $<u\left(c_{1}\right), u\left(c_{2}\right), \ldots, u\left(c_{n}\right)>\in u_{t}(\phi)$;

(g) for all $A, B \in \mathscr{L}_{D}, v_{t}(A \wedge B)=\min \left\{v_{t}(A), v_{t}(B)\right\}$;

(h) for all $A, B \in \mathscr{L}_{D}, v_{t}(A \vee B)=\max \left\{v_{t}(A), v_{t}(B)\right\}$;

(i) for all $A, B \in \mathscr{L}_{D}, v_{t}(A \rightarrow B)=1$ iff $\forall s \succeq t\left[v_{s}(A)=0\right.$ or $\left.v_{s}(B)=1\right]$;

(j) for all $A \in \mathscr{L}_{D}, v_{t}(\neg A)=1$ iff $\forall s \succeq t\left[v_{s}(A)=0\right]$;

(k) in the case of an existential generalization $\exists v A, v_{t}(\exists v A)=1$ iff $v_{t}(A(v / \bar{d}))=1$ for some $d \in D_{t}$;

(1) in the case of a universal generalization $\forall v A, v_{t}(\forall v A)=1$ iff $\forall s \succeq t$ [ $\forall d \in$ $D_{s} v_{s}(A(v / \bar{d})=1]$.

As before, we say that a formula $A$ is verified at a node $t$ in a model $\langle T, \preceq, D, u>$ just in case $v_{t}(A)=1$ and that a model $<T, \preceq, D, u>$ contains a base node if there is a node $t \in T$ such that, for all $s \in T, s \succeq t$.

Soundness of IQL: where $A$ and the formulas in $\Sigma$ belong to $\mathscr{L}$, if $\Sigma \vdash_{\mathrm{IQL}} A$ then at any node in a Kripke model $\langle T, \preceq, D, u>$ at which every member of $\Sigma$ is verified $A$ is verified there too. We note that, where $\mathscr{L}_{t}$ is the extension of $\mathscr{L}$ obtained by adding names for all and only the elements of $D_{t}$ to $\mathscr{L}$, the set of formulas verified at a node in a Kripke model constitutes an $\mathscr{L}_{t}$-consistent, $\mathscr{L}_{t}$-prime, $\mathscr{L}_{t}$-witness-complete, $\mathscr{L}_{t}, R I Q\left\lceil\mathscr{L}_{t}\right.$-theory.

3.2.2. Generic completeness theorem Given a set of formulas $\Phi$, when we wish to indicate that some set $R$ of rules is to be restricted in application exactly to the subformulas of $\Phi$ formed using the constants that occur in members of $\Phi$ or in some notional fixed countably infinite set of parameters foreign to $\Phi$, we may write $R\lceil\Phi$.

THEOREM 4 If $\Sigma \nvdash_{R I Q} \vdash(\Sigma \cup\{A\})$ A then there is a Kripke model $\langle T, \preceq, D, u>$ with a base node such that all members of $\Sigma$ are verified at the base node but $A$ is not verified there.

Proof. We take a countably infinite collection $C$ of countably infinite sets of constants foreign to $\Sigma \cup\{A\}$, together with a set of parameters disjoint from all these sets of constants, and let $\Delta$ be the least set of formulas extending $\Sigma \cup\{A\}$ containing subformulas constructed using constants in $\Sigma \cup\{A\}$ and in $C_{0}$, one of the members of $C$. Clauses (i), 
(ii), (iii), and (vi) of the Generic Lindenbaum-Henkin Lemma (GLHL) apply so there is a $\Delta$-consistent, $\Delta$-prime, $\Delta$-witness-complete $\Delta, R I Q\lceil\Delta$-theory $\Gamma$ contained in $\Delta$, extending $\Sigma$, not containing $A$, and with the properties stated in (i) $(a)-(f)$ of 3.1.1.

Given $\Delta$, consider a set of formulas that differs from $\Delta$ only in that the (countable) set used to obtain instances of existential generalizations extends the set used in forming $\Delta$-it is a union of a finite collection, including $C_{0}$, of members of $C$. Call such a set a witness extension of $\Delta$. The nodes of our small Kripke model are those subsets of witnessextensions $\Delta^{\prime}$ of $\Delta$ that extend $\Gamma$ and that are, relative to the appropriate $\Delta^{\prime}, \Delta^{\prime}$-consistent, $\Delta^{\prime}$-prime, $\Delta^{\prime}$-witness-complete $\Delta^{\prime}, R I Q\left\lceil\Delta^{\prime}\right.$-theories. $\Gamma$ is the base node of the model. $\preceq$ is the subset relation. The domain $D_{\Theta}$ associated with the $\Delta^{\prime}, R I Q \uparrow \Delta^{\prime}$-theory-node $\Theta$ comprises those constants used in forming members of $\Delta^{\prime}$ (so, obviously, $D_{\Theta} \subseteq D_{\Xi}$ when $\Theta \preceq \Xi)$; these constants occur autonymously, that is, for any such constant $c, \bar{c}=c$ and $u(c)=c$. $^{28}$ Where $\phi$ is an $n$-ary predicate, we stipulate that the $n$-tuple of constants $<c_{1}, \ldots c_{n}>$ belongs to $u_{\Theta}(\phi)$ just in case $\phi c_{1} c_{2} \ldots c_{n} \in \Theta$.

Claim For all $\Delta^{\prime}, R I Q\left\lceil\Delta^{\prime}\right.$-theory-nodes $\Theta$ in the model and all $B \in \Delta^{\prime}, B$ is verified at $\Theta$ just in case $B \in \Theta$.

As ever, proof is by induction on complexity of formula (number of occurrences of connectives and quantifiers).

The claim holds for atomic formulas:

$v_{\Theta}\left(\phi c_{1} c_{2} \ldots c_{n}\right)=1$ iff $<u\left(c_{1}\right), u\left(c_{2}\right), \ldots, u\left(c_{n}\right)>\in u_{\Theta}(\phi)$ iff $\left\langle c_{1}, c_{2}, \ldots, c_{n}>\in\right.$ $u_{\Theta}(\phi)$ iff $\phi c_{1} c_{2} \ldots c_{n} \in \Theta$.

The induction hypothesis states that the claim holds for all formulas in each witnessextention $\Delta^{\prime}$ up to some given complexity. We consider the next higher level of complexity.

Induction step. The connectives are dealt with as in 2.2.2. We need only consider quantifiers.

Existential quantifiers If $\exists v B \in \Delta^{\prime}$ then the rules of $\exists$-I and $\exists$-E, restricted to formulas in $\Delta^{\prime}$ and parametric instances of such, belong to $R I Q \uparrow \Delta^{\prime}$ and we have that

If $\exists v B \in \Theta$ then, by the $\Delta^{\prime}$-witness-completeness of $\Theta$, for some parameter $c, B(v / c) \in \Theta$; so $c \in D_{\Theta}$ and, by the induction hypothesis, $v_{\Theta}(B(v / c))=1$, whence $v_{\Theta}(\exists v B)=1$.

If $\exists v B \notin \Theta$ then, by $\Theta$ 's $\Delta^{\prime}, R \backslash \Delta^{\prime}$-theoryhood, $\Theta \nvdash_{R \mid \Delta^{\prime}} \exists v B$; as $\exists$-I is one of our rules, there is no constant $c$ in $\Delta^{\prime}$, for which $B(v / c) \in \Theta$; there is, then, no member $c$ of $D_{\Theta}$ for which $v_{\Theta}(B(v / c))=1$, and hence $v_{\Theta}(\exists v B)=0$.

Universal quantifiers If $\forall v B \in \Delta^{\prime}$ then the rules of $\forall$-I and $\forall$-E, restricted to formulas in $\Delta^{\prime}$ and parametric instances of such, belong to $R I Q \uparrow \Delta^{\prime}$ and we have that

If $\forall v B \in \Theta$ then, for all $\Delta^{\prime \prime}, R I Q\left\lceil\Delta^{\prime \prime}\right.$-theories $\Xi$ extending $\Theta, \forall v B \in$ $\Xi$-here $\Delta^{\prime \prime}$ differs from $\Delta^{\prime}$ only in that more witnesses may be present; for any constant $c$ in $\Delta^{\prime \prime}$, the rule $\forall$-E, applied only to formulas in $\Delta^{\prime \prime}$, ensures that $\Xi \vdash_{R \mid \Delta^{\prime \prime}} B(v / c)$; by the $\Delta^{\prime \prime}, R I Q,\left\lceil\Delta^{\prime \prime}\right.$-theoryhood of $\Xi$, for all such constants $c, B(v / c) \in \Xi$; by the induction hypothesis, we have that $\forall \Xi \supseteq \Theta\left[\forall c \in D_{\Xi}, v_{\Xi}(B(v / c))=1\right]$ and so $v_{\Theta}(\forall v B)=1$.

If $\forall v B \notin \Theta$ then, by the $\Delta^{\prime}, R I Q \backslash \Delta^{\prime}$-theoryhood of $\Theta, \Theta \nvdash_{R \mid \Delta^{\prime}} \forall v B$; let $a$ be a constant foreign to $\Delta^{\prime}$ - we know there are such available for

28 By design, $D_{\Gamma}$ is nonempty, indeed countably infinite. 
the constants in $\Delta^{\prime}$ belong to only finitely many of the members of $C$; $\Theta \nvdash_{R \vdash\left(\Delta^{\prime} \cup\{B(v / a)\}\right)} B(v / a)$, for, $a$ being foreign to $\Delta^{\prime}$, were there to be a $R \uparrow\left(\Delta^{\prime} \cup\{B(v / a)\}\right)$-proof of $B(v / a)$ from $\Theta$, as $a$ would behave essentially as a parameter, by an application of $\forall$-I, we would have a $R\left\lceil\Delta^{\prime}\right.$ proof of $\forall v B$ from $\Theta$, which we know there not to be; let $\Delta^{\prime \prime}$ expand $\Delta^{\prime}$ to include all subformulas employing the extra constants belonging to the same member of $C$ as $a$; by the GLHL there's a $\Delta^{\prime \prime}$-consistent, $\Delta^{\prime \prime}$ prime, $\Delta^{\prime \prime}$-witness-complete $\Delta^{\prime \prime}, R I Q \mid \Delta^{\prime \prime}$-theory $\Xi$, extending $\Theta$ and not containing $B(v / a) ; \Theta \preceq \Xi, a \in D_{\Xi}$ and, by the induction hypothesis, $v_{\Xi}(B(v / a))=0$, hence $v_{\Theta}(\forall v B)=0$.

3.2.3. Subformula and separation properties for $I Q L$ As an immediate consequence of Theorems 4 and 2.5 we have that IQL has the subformula and separation properties.

3.2.4. Subformula and separation properties for $M Q L$ (minimal first-order logic) As MPL is to IPL, so is MQL to IQL: it is obtained by dropping $\neg$-E from IQL; its Kripke models are quintuples $<T, \preceq, D, u, \mathscr{F}>$ with $\mathscr{F}_{\text {an }} \preceq$-open set in $T$ (i.e., if $t \in \mathscr{F}$ and $t \preceq s$ then $s \in \mathscr{F}$ ) and negation is evaluated as in Kripke models for MPL. Just as in 2.3, we find that MQL has the subformula property: if $\Sigma \vdash_{\mathrm{MQL}} A$ then $\Sigma \vdash_{R M Q(\Sigma \cup\{A\})} A$.

\subsection{Some results that build on $\$ 2$ and 3.1 and 3.2.}

3.3.1. The $\{\wedge, \vee, \rightarrow, \forall, \exists\}$-fragment of the intermediate logic $K Q L$

TheOREM 5 If $C(\Sigma \cup\{A\}) \subseteq\{\wedge, \vee, \rightarrow, \forall, \exists\}$ and $\Sigma \vdash_{\mathrm{KQL}} A$ then $\Sigma \vdash_{\mathrm{IQL}} A$.

— The "supernode" contains all formulas in the countably many extensions of $\Delta_{0}$.

\subsubsection{Subformula and separation theorems for the $\{\wedge, \vee, \exists\}$-fragment of $C Q L$}

Theorem 6 If $\Sigma \vdash_{\mathrm{CQL}} A$ and $C(\Sigma \cup\{A\}) \subseteq\{\wedge, \vee, \exists\}$ then $\Sigma \vdash_{R M Q(\Sigma \cup\{A\})} A$.

— GLHL adds $\Delta$-witness-completeness to Theorem 2.9.

3.3.3. A weak subformula property for the $\{\wedge, \vee, \neg, \exists\}$-fragment of $C Q L$ as $M Q L+D N E$ In the derivation of any $\rightarrow$-free, $\forall$-free sequent $\Sigma \vdash A$ that is classically valid but not derivable in MQL, only subformulas of members of $\Sigma \cup\{\neg \neg A\}$ need occur. DNE, which must be used in deriving the sequent, need be used only once and we may further insist that its application be the last step. The $\{\wedge, \vee, \neg, \exists\}$-fragment of MQL + DNE has the separation property.

3.3.4. A weak subformula property for the $\{\wedge, \vee, \rightarrow, \neg, \exists\}$-fragment of $C Q L$ as $I Q L+$ $D N E$ In the derivation of any $\forall$-free sequent $\Sigma \vdash A$ that is classically valid but not derivable in IQL, only subformulas of members of $\Sigma \cup\{\neg \neg A\}$ need occur. DNE, which must be used in deriving the sequent, need be used only once and we may further insist that its application be the last step.

3.3.5. A weak subformula property for the $\{\wedge, \vee, \rightarrow, \neg, \exists\}$-fragment of $C Q L$ as $I Q L+$ $C R A$ or IQL + Dilemma In the derivation of any $\forall$-free sequent $\Sigma \vdash A$ that is classically valid but not derivable in IQL, only subformulas of members of $\Sigma \cup\{\neg A\}$ need occur. CRA or Dilemma, one of which must be used in deriving the sequent, need be used only once and we may further insist that, whichever we use, its application be the last step. 
3.3.6. The $\{\wedge, \vee, \neg, \exists\}$-fragment of $C Q L$ as $I Q L+D$ ilemma has the subformula property

3.3.7. The $\{\wedge, \vee, \rightarrow, \exists\}$-fragment of $C Q L$ as $I Q L+$ Peirce's Rule has the separation property

3.3.8. The $\{\wedge, \vee, \rightarrow, \exists\}$-fragment of $C Q L$ as $I Q L+$ Tarski's Rule has the subformula property

3.3.9. A formulation of the $\{\wedge, \vee, \rightarrow, \neg, \exists\}$-fragment of $C Q L$ with the (strict) subformula property and no redundancy of rules The rules are: $\wedge$-I, $\wedge$-E, $\vee-\mathrm{I}, \vee-\mathrm{E}$, weak $\rightarrow-\mathrm{I}$, Tarski's Rule, $\rightarrow$-E, Dilemma, $\neg$-E, $\exists$-I, and $\exists$-E.

3.3.10. The shape of things to come Adding the standard introduction and elimination rules for the universal quantifier to those listed immediately above yields a formulation of CQL. That the sequent $\forall x(F x \vee G a) \vdash \forall x F x \vee G a$ is classically valid but not intuitionistically so shows, however, that even the separation property fails for this formulation. Consequently, if we are to achieve a formulation of CQL with the subformula property that builds on what we have obtained so far, we cannot make do with the standard rules for both disjunction and the universal quantifier.

3.4. Keeping small Kripke models small. In the construction used in the proof of the GLHL in 3.1.1 we help ourselves to a countable infinity of additional names and use them in forming a stock of substitution instances of quantified formulas in $\Sigma \cup\{A\}$. This has the effect that though the number of nodes involved in any model may be small, the domain at each node is countably infinite. One might, with some justice, complain, as a referee did, that the models are no longer small. A first step - and the only one to be taken here-in the direction of keeping models small is to alter as follows the basic construction used in proof of the GLHL when the initial set of formulas $\Sigma \cup\{A\}$ is finite.

$\Delta_{0}$ contains all subformulas of $\Sigma \cup\{A\}$ obtained using names occurring in $\Sigma \cup\{A\}$, if there are any, and all subformulas formed using one new name, if there is none. Additional names are added as needed, need being determined by the details of the LindenbaumHenkin construction, details we amend as follows.

Taking note of the fact that $\Delta_{0}$ is finite, we let $B_{0}, B_{1}, \ldots, B_{k}$ be an initial enumeration of the formulas in $\Delta_{0}$. We define a sequence of extensions of $\Sigma$ as follows:

(A) $\Gamma_{0}=\Sigma ; I_{0}=\{0,1, \ldots, k\}$;

if $n+1 \notin I_{n}$, stop and set $\Gamma=\cup_{m \in I_{n}} \Gamma_{m}$;

otherwise

(B) $\Gamma_{n+1}=\Gamma_{n}$ if $\Gamma_{n}, B_{n} \vdash_{R \backslash \Delta} A, I_{n+1}=I_{n}$;

$\Gamma_{n+1}=\Gamma_{n} \cup\left\{B_{n}\right\}$ if $\Gamma_{n}, B_{n} \nvdash_{R \backslash \Delta} A$ and $B_{n}$ is not an existential generalization,

$I_{n+1}=I_{n}$

$\Gamma_{n+1}=\Gamma_{n} \cup\{\exists v D, D(v / c)\}$, where $D(v / c)$ is an instance employing a constant not occurring in $\Gamma_{n} \cup\left\{B_{n}, A\right\}$, if $B_{n}$ is the formula $\exists v D$ and $\Gamma_{n}, B_{n} \nvdash_{R \backslash \Delta} A$; in this last case, add $B_{j+1}, B_{j+2}, \ldots, B_{m}$ to the current enumeration of formulas $B_{0}, B_{1}, \ldots, B_{j}$, where $j=\max I_{n}$ and $B_{j+1}, B_{j+2}, \ldots, B_{m}$ are substitution instances of quantified formulas in $\left\{B_{i}: i \in I_{n}\right\}$ created using the new constant $c$ and whatever constants already occur in $\left\{B_{i}: i \in I_{n}\right\} . I_{n+1}=\{0,1, \ldots, j, j+1, \ldots, m\}$.

(C) $\Gamma=\cup_{n \in \mathbb{N}} \Gamma_{n}$ if for no $n \in \mathbb{N}$ is it the case that $n+1 \notin I_{n}$.

In some cases this works beautifully to yield the smallest possible Kripke model. For example, $\forall x(F x \vee G a) \nvdash_{\mathrm{IQL}} \forall x F x \vee G a$ : the construction delivers a model of two nodes; the base node contains a single element, denoted by $a$, which, at the base node, is in the extension of $F$ but not of $G$; the second node has a domain of two elements, one being the 
denotation of $a$, which at this node is in the extension of $G$, and in the extension of $F$, but the other element of the domain is not in the extension of $F$.

In some cases the method delivers small but not the smallest possible models exactly because the way built in to the construction of Kripke models to have a universally quantified formula fail to be verified at the base node is to introduce a new node (see 3.2.2). So in applying the method to the classically invalid $\exists x F x \vdash \forall x F x$ we obtain a model with two nodes. Like the previous model, the base node has a domain containing one element that falls within the extension of $F$ there, the second node contains an additional element that does not fall in the extension of $F$ at that node.

Comparably to what happens with semantic tableaux/trees, where the amended method falls down badly is in the case of mixed quantification and relations. Showing the classically invalid quantifier-shift fallacy-from $\forall x \exists y R x y$ to $\exists y \forall x R x y$ - to be fallacious, the amended method yields a model with a base node with a countably infinite domain in which all pairs of elements are in the extension of $R$, the base node having a countably infinity of distinct successor nodes, one for each element in the domain of the base node, each branching off on its own; for each element $a$ in the domain of the base node, in its successor node (if I may put it that way) there is some new element not in the base node, $d_{a}$, say, for which the pair $\left\langle d_{a}, a\right\rangle$ is not in the extension of $R$ at that node.

Doubtless one could attempt to refine the construction in the proof of the GLHL (cf. Boolos, 1984). But whether the game is worth the candle is moot. At some point systematicity and the desire for small models with small domains run up against undecidability considerations. And there is a definite cost in terms of the simplicity of the construction. In what follows no attempt will be made to keep domains small.

3.5. Strong $\Delta$-counter-witness-completeness. Given a set $\Delta$ of formulas of a language $\mathscr{L}$ we say that a set of formulas $\Gamma$ is $\Delta$-counter-witness-complete if

for all universal generalizations $\forall v A$ in $\Delta, \forall v A \in \Gamma$ if $A(v / c) \in \Gamma$ for all constants $c$ in $\mathscr{L}$.

Absence, for some name $c$ in $\mathscr{L}$, of $A(v / c)$ from $\Gamma$ then provides a witness to $\Gamma$ 's failure to endorse the universal generalization $\forall v A$. A more forceful marker-assuming consistency of $\Gamma$-than mere failure on $\Gamma$ 's part to affirm $A(v / c)$ is presented when $\Gamma$ affirms the contrary of $A(v / c)$.

3.5.1. Contraries If $A=\neg B$, for some formula $B$, then $-A$, the contrary of $A$, is $B$; otherwise $-A=\neg A$.

\subsubsection{Generic Lindenbaum-Henkin Lemma: first extension}

THEOREM 7 Given $R$, when $\Sigma \nvdash_{R} A$ and where $c_{0}, c_{1}, \ldots, c_{n}, \ldots$, is an infinite sequence of constants all foreign to $\Sigma \cup\{A\}$ let $\Delta_{0}$ contain all subformulas of members of $\Sigma \cup\{A\}$ formed using $c_{0}, c_{1}, \ldots, c_{n}, \ldots$ and whatever other constants may occur in members of $\Delta_{0}$. Let $\Delta_{1}$ be the smallest extension of $\Delta_{0}$ that contains $-A$ and, for each constant $d$ in $\Delta_{0}$, contains $-D(v / d)$ when $\forall v D \in \Delta_{0}$. Let $\Delta_{1} \subseteq \Delta$ and, as usual, let $R \uparrow \Delta$ stand for the restriction of the rules in $R$ obtained by excluding rules governing those connectives and quantifiers that do not occur in any member of $\Delta$ and restricting those that remain to formulas in $\Delta$ and parametric instances of these. By design, $\neg$ occurs in formulas in $\Delta_{1}$. If $\Delta_{0}=\Delta_{1}$ and the rules $\neg-I$ and $\neg-E$ either belong to $R$ or are safely $\Delta$-derived rules given the rules in $R$, or if $\Delta_{0} \varsubsetneqq \Delta_{1}$ and the rules $\neg-I$, Dilemma, and $\neg-E$ either belong to $R$ or are safely $\Delta$-derived rules given the rules in $R$, then 
(i) there is a well-behaved $\Delta_{1}, R \backslash \Delta$-theory $\Gamma$ such that $\Sigma \subseteq \Gamma \subseteq \Delta_{1}, A \notin \Gamma$, and (a) for all formulas $B$ in $\Delta_{1}, B \notin \Gamma$ if, and only if, $\Gamma, B \vdash_{R \mid \Delta} A$;

(ii) if $\vee$ occurs in $\Delta_{1}$ and the rules of $\vee$-I and $\vee$-E either belong to $R$ or are safely $\Delta$-derived rules given the rules in $R, \Gamma$ is $\Delta_{1}$-prime;

(iii) $\Gamma$ is $\Delta_{1}$-consistent;

(iv) $\Gamma$ is $\Delta_{1}$-negation-complete;

(v) if $\rightarrow$ occurs in $\Delta_{1}$ and the rule of $\rightarrow-$-I either belongs to $R$ or is a safely $\Delta$-derived rule given the rules in $R, \Gamma$ is $\Delta_{1}$-implication-complete;

$\left(v i_{1}\right)$ if an existential generalization belongs to $\Delta_{1}$ and the rule of $\exists-E$ belongs to $R$ or is a safely $\Delta$-derived rule given the rules in $R$ then $\Gamma$ is $\Delta_{1}$-witness-complete;

$\left(v i_{2}\right)$ if a universal generalization belongs to $\Delta_{1}$ and the rule of $\forall-I$ belongs to $R$ or is a safely $\Delta$-derived rule given the rules in $R$ then $\Gamma$ is $\Delta_{1}$-counter-witness-complete.

Proof. Let $B_{0}, B_{1}, \ldots, B_{n}, \ldots$ be an enumeration of the formulas in $\Delta_{0}$, an enumeration indexed by $\mathbb{N}$. We define a sequence of extensions of $\Sigma$ as follows:

(A) $\Gamma_{0}=\Sigma$;

for all $n \in \mathbb{N}$,

(B) $\Gamma_{n+1}=\Gamma_{n} \cup\left\{B_{n}\right\}$ if $\Gamma_{n}, B_{n} \nvdash_{R \backslash \Delta} A$ and $B_{n}$ is not an existential generalization,

$\Gamma_{n+1}=\Gamma_{n}$ if $\Gamma_{n}, B_{n} \vdash_{R \backslash \Delta} A$ and $B_{n}$ is not a universal generalization,

$\Gamma_{n+1}=\Gamma_{n} \cup\{\exists v D, D(v / c)\}$, where $D(v / c)$ is an instance employing a constant not occurring in $\Gamma_{n} \cup\left\{B_{n}, A\right\}$, if $B_{n}$ is the formula $\exists v D$ and $\Gamma_{n}, B_{n} \nvdash_{R \backslash \Delta} A$,

$\Gamma_{n+1}=\Gamma_{n} \cup\{-D(v / c)\}$, where $D(v / c)$ is an instance employing a constant not occurring in $\Gamma_{n} \cup\left\{B_{n}, A\right\}$, if $B_{n}$ is the formula $\forall v D$ and $\Gamma_{n}, B_{n} \vdash_{R \vdash \Delta} A$.

We set $\Gamma=\cup_{n \in \mathbb{N}} \Gamma_{n}$ and show, for all $n \in \mathbb{N}$, that $\Gamma_{n} \nvdash_{R \backslash \Delta} A$. As ever, the proof goes by induction. Supplementary to what has been done above, we must show that $\Gamma_{n+1} \nvdash_{R \vdash \Delta} A$ when $\Gamma_{n+1}=\Gamma_{n} \cup\{-D(v / c)\}$, where $c$ is foreign to $\Gamma n \cup\{\forall v D, A\}$, because $\Gamma_{n}, \forall v D$ $\left(=B_{n}\right) \vdash_{R \backslash \Delta} A$.

Suppose that $\Gamma_{n},-D(v / c) \vdash_{R \backslash \Delta} A$. Then, by $\neg-\mathrm{E}, \Gamma_{n},-A,-D(v / c) \vdash_{R \backslash \Delta} D(v / c)$. Whence, by Dilemma if $-D(v / d)=\neg D(v / d)$ and by $\neg$-I if not, $\Gamma_{n},-A \vdash_{R \backslash \Delta} D(v / c)$. As $c$ is foreign to $\Gamma_{n} \cup\{A\}, \Gamma_{n},-A \vdash_{R \backslash \Delta} \forall v D$, whence $\Gamma_{n},-A \vdash_{R \backslash \Delta} A$. By Dilemma if $-A=\neg A$ and by $\neg$-I if not, $\Gamma_{n} \vdash_{R \backslash \Delta} A$, which, by the appropriate inductive hypothesis, we know not to be the case.

(iii) $\neg$-E gives us $\Delta_{1}$-consistency.

(iv) if $-A=\neg A$, Dilemma secures $\Delta_{1}$-negation-completeness; otherwise $\neg$-I suffices.

(v) if $B \in \Delta_{1}, B \rightarrow C \in \Delta_{1}, B \notin \Gamma$, and $B \rightarrow C \notin \Gamma$, then $\Gamma, B \vdash_{R \mid \Delta} A$ and $\Gamma, B \rightarrow C \vdash_{R \backslash \Delta} A$. By $\neg-\mathrm{E}, \Gamma,-A, B \vdash_{R \backslash \Delta} C$, whence, by $\rightarrow-\mathrm{I}, \Gamma,-A-\vdash_{R \backslash \Delta}$ $B \rightarrow C$. But then $\Gamma,-A \vdash_{R \triangleright \Delta} A$. Finally, by Dilemma if $-A=\neg A$ and by $\neg-\mathrm{I}$ otherwise, $\Gamma \vdash_{R \triangleright \Delta} A$, which we know not to be the case.

(vi 2 ) If $\forall v D \notin \Gamma$, then $\Gamma_{n}, \forall v D \vdash_{R \vdash \Delta} A$, where $\forall v D=B_{n}$. Hence $-D(v / c) \in \Gamma_{n+1} \subseteq \Gamma$. By $\Delta_{1}$-consistency of $\Gamma, D(v / c) \notin \Gamma$.

3.5.3. Weak subformula property for CQL as IQL + Dilemma and IQL + CRA Attending to the details of the proof in the previous section, we find that we can do better. Making use of the observation at 2.5.3.1, we see that in the presence of $\neg$-I, the only applications of Dilemma/CRA that we need in order to establish negation-completeness deduce $A$ from a subproof in which $A$ is deduced from its contrary-and clearly we only 
need Dilemma/CRA in case $-A=\neg A$, for otherwise $\neg$-I suffices. Consequently we can restrict the possible applications permitted of Dilemma or CRA to obtain:

THEOREM 8 When CQL is formulated as IQL + Dilemma, then when $\Sigma \vdash_{\mathrm{CQL}}$ A there is a proof in which, over and above subformulas of members of $\Sigma \cup\{A\}$, only $\neg A$ and negations of instances of universal generalizations that are themselves subformulas of members of $\Sigma \cup\{A\}$ occur. But $\neg$ A need not occur if A itself has negation dominant, and, likewise, the negation $\neg D(v / c)$ of an instance of a subformula $\forall v D$ need not occur if $D$ has negation dominant. Furthermore, those nonsubformulas that do occur need occur only as assumptions discharged in applications of Dilemma and these are the only applications of Dilemma in the proof. Lastly, if a nonsubformula that is the negation $\neg D(v / c)$ of an instance of a subformula $\forall v D$ does so occur, it occurs in an application of Dilemma whose immediate conclusion is $D(v / c)$, and, likewise, if $\neg A$ does occur as an assumption discharged in an application of Dilemma, it is an application whose immediate conclusion is A. Such applications of Dilemma are, in effect, applications of CRA, for the subproof that "does the work" is a derivation of a formula in which its own negation occurs as assumption.

This is an optimal strengthening of the subformula results obtained by Prawitz (1963, secs. 3.1, 3.2, 4.1, and 4.2), for the $\vee$ - and $\exists$-free fragment of CQL, and by Stålmarck (1991), for full CQL, as consequences of normalization theorems. Optimality is shown by two considerations. (1) We cannot make do with only the negations of instances of universal generalizations that are subformulas, for there are $\forall$-free, $\neg$-free classically valid, intuitionistically invalid inferences. (2) We cannot dispense with all the negations of instances of universal generalizations that are subformulas other than the negation of the conclusion, for if we could we would obtain a fallacious strong Glivenko Theorem: we would have that $\Sigma \vdash_{\mathrm{CQL}} \neg A$ only if $\Sigma \vdash_{\mathrm{IQL}} \neg A$. But this is well known to be false. The standard counterexample:

$\forall x \neg \neg F x \vdash_{\mathrm{CQL}} \neg \neg \forall x F x$ but $\{\forall x \neg \neg F x, \neg \forall x F x\}$ is intuitionistically consistent.

3.5.3.1. Weak subformula property for CQL as IQL + Peirce's Rule Zimmermann (2002) has obtained a normalization theorem for classical first-order logic formulated with a falsum constant as primitive (and negation a mere abbreviatory device) and Peirce's Rule, taken, following Curry (1963), as an extra elimination rule for the conditional. ${ }^{29}$ In this setting, bearing the immediately above and Theorem 2.19 in mind, it is not difficult to show

THEOREM 9 When $\Sigma \vdash_{\mathrm{CQL}} A$ there is a proof in which, over and above subformulas of members of $\Sigma \cup\{A\}$, only $A \rightarrow \perp$, conditionals of the form $A \rightarrow C$ where $C$ is the consequent of a conditional that is a subformula of a member of $\Sigma \cup\{A\}$, and conditionals of the form $D(v / c) \rightarrow \perp$ where $D(v / c)$ is an instance of a universal generalization that is a subformula of a member of $\Sigma \cup\{A\}$ occur. But $A \rightarrow \perp$ need not occur if $A$ itself is of the form $E \rightarrow \perp$, and, likewise, the conditional $D(v / c) \rightarrow \perp$ involving an instance of a subformula $\forall v D$ need not occur if $D$ is of the form $E \rightarrow \perp$. Furthermore, those nonsubformulas that do occur need occur only as assumptions discharged in applications of Peirce's Rule and these are the only applications of Peirce's Rule in the proof.

3.5.4. Strong Glivenko Theorem for CQL From Theorem 7 we see that when the conclusion $A$ of the underivable sequent $\Sigma \vdash A$ is of the form $\neg E$, for some $E, \Gamma$ is

29 I owe this reference to Roy Dyckhoff. 
$\Delta_{1}$-negation- and $\Delta_{1}$-implication-complete and the only appeals to Dilemma in the proof of the extension of GLHL occur in showing that $\Gamma_{n} \vdash_{R \backslash \Delta} \neg E$ when $\Gamma_{n}, \forall v D\left(=B_{n}\right) \vdash_{R \mid \Delta}$ $\neg E$ and $\Gamma_{n},-D(v / c) \vdash_{R \backslash \Delta} \neg E$ (where $c$ does not occur $\Gamma_{n} \cup\{\forall v D, E\}$ ). The rule that most directly establishes this is

MH

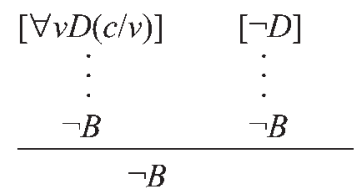

subject to the restrictions that $c$ not occur in any assumption other than $\neg D$ and $c$ not occur in $B$.

MH is an inessential variant of Seldin's (1986) Mh. As an addition to IQL it is equivalent to the Double Negation Shift rule (cf. Seldin,1986)

DNS

$$
\frac{\forall v \neg \neg D}{\neg \neg \forall v D}
$$

DNS is the most obvious rule to add to IQL in order to render $\{\forall x \neg \neg F x, \neg \forall x F x\}$ inconsistent. By adding $\mathrm{MH}$ in its place and adapting the proof of Theorem 7 we obtain an elegant proof of

THEOREM 10 If $\Sigma \vdash_{\mathrm{CQL}} \neg A$ then $\Sigma \vdash_{\mathrm{IQL}+\mathrm{MH}} \neg A$.

We also obtain information regarding subformulas. When $\Sigma \vdash_{\mathrm{CQL}} \neg A$ there is a proof of $\neg A$ from $\Sigma$ in IQL + MH in which, over and above subformulas of members of $\Sigma \cup\{\neg A\}$, only negations of instances of universal generalizations that are themselves subformulas of members of $\Sigma \cup\{\neg A\}$ occur. But the negation $\neg D(v / c)$ of an instance of a subformula $\forall v D$ need not occur if $D$ has negation dominant. Furthermore, those nonsubformulas that do occur need occur only as assumptions discharged in applications of $\mathrm{MH}$ and these are the only applications of $\mathrm{MH}$ in the proof. Lastly, if a nonsubformula that is the negation $\neg D(v / c)$ of an instance of a subformula $\forall v D$ does so occur, it occurs in an application of MH whose immediate conclusion is $\neg A$.

3.6. Weak $\Delta$-counter-witness-completeness. The Kripke semantics for first-order IQL (and MQL) allows domains to grow: it is required only that, for any nodes $s$ and $t$ in a model $<T, \preceq, D, u>, D_{s} \subseteq D_{t}$ when $s \preceq t$. If we confine attention to those models in which the domain is constant, that is, $\forall s, t \in T D_{s}=D_{t}(\neq \varnothing)$, then, given the other properties of Kripke models, we can simplify radically the verification conditions at a node for universal generalizations:

$$
\left(\mathrm{l}_{\mathrm{CD}}\right) v_{t}(\forall v A)=1 \text { iff }\left[\forall d \in D v_{t}(A(v / \bar{d}))=1\right],
$$

where $D$ is the (nonempty) domain common to all nodes in the model. If we are to mimic this condition in the theories we obtain via the Lindenbaum-Henkin construction, we must aim at the very least for $\Delta$-counter-witness-completeness: we want a Lindenbaum-Henkinstyle construction to deliver a base node that is $\Delta_{0}$-counter-witness-complete.

\subsubsection{The Generic Lindenbaum-Henkin Lemma: second extension}

THEOREM 11 Given $R$, when $\Sigma \nvdash_{R} A$ and where $c_{0}, c_{1}, \ldots, c_{n}, \ldots$, is an infinite sequence of constants all foreign to $\Sigma \cup\{A\}$ let $\Delta_{0}$ contain all subformulas of members 
of $\Sigma \cup\{A\}$ formed using $c_{0}, c_{1}, \ldots, c_{n}, \ldots$ and whatever other constants may occur in members of $\Sigma \cup\{A\}$. Let $\Delta_{0} \subseteq \Delta$, and let $R \backslash \Delta$ stand for the restriction of the rules in $R$ obtained by excluding rules governing those connectives and quantifiers that do not occur in any member of $\Delta$ and restricting those that remain to formulas in $\Delta$ and parametric instances of these. Then, keeping the lettering and numbering of clauses from previous sections,

(i) there is a well-behaved $\Delta_{0}, R \backslash \Delta$-theory $\Gamma$ such that $\Sigma \subseteq \Gamma \subseteq \Delta_{0}, A \notin \Gamma$;

(iii) if $\neg$ occurs in $\Delta_{0}$ and the rule of $\neg$-E either belongs to $R$ or is a safely $\Delta$-derived rule given the rules in $R, \Gamma$ is $\Delta_{0}$-consistent;

(iv $v_{1}$ ) if $A=\neg D$, for some $D \in \Delta_{0}$ and the rule of weak $\neg$-I either belongs to $R$ or is a safely $\Delta$-derived rule given the rules in $R, \Gamma$ is $\Delta_{0}$-consistent;

$\left(v i_{1}\right)$ if an existential generalization belongs to $\Delta_{0}$ and the rule of $\exists$-E belongs to $R$ or is a safely $\Delta$-derived rule given the rules in $R$ then $\Gamma$ is $\Delta_{0}$-witness-complete;

(vi $i_{2}$ ) if a universal generalization belongs to $\Delta_{0}$ and the rule of $\forall-I$ belongs to $R$ or is a safely $\Delta$-derived rule given the rules in $R$ then $\Gamma$ is $\Delta_{0}$-counter-witnesscomplete.

Proof. Let $B_{0}, B_{1}, \ldots, B_{n}, \ldots$ be an enumeration of the formulas in $\Delta_{0}$, an enumeration indexed by $\mathbb{N}$. We define a sequence of extensions of $\Sigma$ and a sequence of extensions of $\{A\}$ as follows:

(A) $\Gamma_{0}=\Sigma ; Z_{0}=\{A\}$; for all $n \in \mathbb{N}$,

(B) $\Gamma_{n+1}=\Gamma_{n} \cup\left\{B_{n}\right\}, Z_{n+1}=Z_{n}$ if $B_{n}$ is not an existential generalization and for no $C \in \mathrm{Z}_{n}$ is it the case that $\Gamma_{n}, B_{n} \vdash_{R \mid \Delta} C$,

$\Gamma_{n+1}=\Gamma_{n}, \mathrm{Z}_{n+1}=\mathrm{Z}_{n}$ if $\Gamma_{n}, B_{n} \vdash_{R \mid \Delta} C$, for some $C \in \mathrm{Z}_{n}$, and $B_{n}$ is not a universal generalization,

$\Gamma_{n+1}=\Gamma_{n} \cup\{\exists v D, D(v / c)\}$, where $D(v / c)$ is an instance employing a constant not occurring in $\Gamma_{n} \cup \mathrm{Z}_{n} \cup\left\{B_{n}\right\}, \mathrm{Z}_{n+1}=\mathrm{Z}_{n}$ if $B_{n}$ is the formula $\exists v D$ and for no $C \in \mathrm{Z}_{n}$ is it the case that $\Gamma_{n}, B_{n} \vdash_{R \mid \Delta} C$,

$\Gamma_{n+1}=\Gamma_{n}, \mathrm{Z}_{n+1}=\mathrm{Z}_{n} \cup\{D(v / c)\}$, where $D(v / c)$ is an instance employing a constant not occurring in $\Gamma_{n} \cup Z_{n} \cup\left\{B_{n}\right\}$, if $B_{n}$ is the formula $\forall v D$ and $\Gamma_{n}, B_{n} \vdash_{R \mid \Delta} C$, for some $C \in \mathrm{Z}_{n}$.

We set $\Gamma=\cup_{n \in \mathbb{N}} \Gamma_{n}, Z=\cup_{n \in \mathbb{N}} Z_{n}$.

First we show, for all $n \in \mathbb{N}$, that $\Gamma_{n} \nvdash_{R \backslash \Delta} C$ for any $C \in \mathrm{Z}_{n}$.

True by hypothesis for $n=0$. Induction hypothesis (IH): suppose that it's true for $n=k$. Cases:

(1) $\Gamma_{k+1}=\Gamma_{k} \cup\left\{B_{k}\right\}, Z_{k+1}=Z_{k}$ and $B_{k}$ is not an existential generalization: if $\Gamma_{k}$, $B_{k} \vdash_{R \backslash \Delta} C$ for some $C \in Z_{k}$, then, by construction, $\Gamma_{k+1}=\Gamma_{k}$ and hence, contrary to IH, $\Gamma_{k} \vdash_{R \mid \Delta} C$ for some $C \in \mathrm{Z}_{k}$;

(2) $\Gamma_{k+1}=\Gamma_{k}, Z_{k+1}=Z_{k}$ and $B_{k}$ is not a universal generalization: by $\mathrm{IH}, \Gamma_{k+1}=$ $\Gamma_{k} \nvdash_{R \mid \Delta} C$, for any $C \in \mathrm{Z}_{k}=\mathrm{Z}_{k+1}$;

(3) $\Gamma_{k+1}=\Gamma_{k} \cup\{\exists v D, D(v / c)\}$, where $D(v / c)$ is an instance employing a constant not occurring in $\Gamma_{k} \cup Z_{k} \cup\left\{B_{k}\right\}, Z_{k+1}=Z_{k}$ and $B_{k}$ is the formula $\exists v D$ : if $\Gamma_{k}, B_{k}, D(v / c) \vdash_{R \mid \Delta} C$ for some $C \in Z_{k}$, then, as $c$ is foreign to $\Gamma_{k} \cup Z_{k} \cup\left\{B_{k}\right\}$, we have that $\Gamma_{k}, B_{k}, \exists v D \vdash_{R \mid \Delta} C$, that is, $\Gamma_{k}, B_{k} \vdash_{R \mid \Delta} C$, for some $C \in \mathrm{Z}_{k}$, and the argument now proceeds as in (1); 
(4) $\Gamma_{k+1}=\Gamma_{k}, Z_{k+1}=Z_{k} \cup\{D(v / c)\}$, where $D(v / c)$ is an instance employing a constant not occurring in $\Gamma_{k} \cup\left\{B_{k}\right\}$, and $B_{k}$ is the formula $\forall v D$ : suppose that $\Gamma_{k} \vdash_{R / \Delta} D(v / c)$, IH ruling out any other possibility; by $\mathrm{IH}, D(v / c) \notin \mathrm{Z}_{k}$; the only possibility is that $\Gamma_{k}, \forall v D \vdash_{R \mid \Delta} C$, for some $C \in \mathrm{Z}_{k}$; as $c$ is foreign to $\Gamma_{k} \cup \mathrm{Z}_{k} \cup\{\forall v D\}, \Gamma_{k} \vdash_{R \mid \Delta} \forall v D$ and hence $\Gamma_{k} \vdash_{R \backslash \Delta} C$, for some $C \in \mathrm{Z}_{k}$, contrary to IH.

Consequently, if $C \in \mathrm{Z}$ then $\Gamma \nvdash_{R \mid \Delta} C$, for if, on the contrary, $C \in \mathrm{Z}_{k}$, for some $k \in \mathbb{N}$, then, proofs being finite, for some $m \in \mathbb{N}, \Gamma_{m} \vdash_{R \backslash \Delta} C$; letting $n=\max \{k, m\}$, we contradict what has just been shown. It follows that $\Gamma$ is a $\Delta_{0}, R \backslash \Delta$-theory.

(iii) If $\neg$ occurs in $\Delta_{0}$ and $R$ gives us the rule of $\neg$-E, $\Gamma$ is $\Delta_{0}$-consistent: if $B \in \Delta_{0}$, $\neg B \in \Delta_{0}, B \in \Gamma$, and $\neg B \in \Gamma$ then, by $\neg$-E, $\Gamma \vdash_{R \mid \Delta} C$, for all $C \in \mathrm{Z}$, which we know not to be the case.

(iv 1 ) If $A=\neg D$, and $R$ gives us the rule $\neg$-inv, $\Gamma$ is $\Delta_{0}$-consistent: if $B \in \Delta_{0}, \neg B \in$ $\Delta_{0}, B \in \Gamma$, and $\neg B \in \Gamma$ then, by weak $\neg$-I, $\Gamma \vdash_{R \mid \Delta} \neg D$, and, as $\neg D \in \mathrm{Z}$, we know this not to be the case.

(vi) $\Delta_{0}$-witness-completeness and $\Delta_{0}$-counter-witness-completeness are built into the construction (given the $\Delta_{0}, R \backslash \Delta$-theoryhood of $\Gamma$ ).

Notice the complete absence of mention of disjunctions and therewith primeness of $\Delta_{0}$. The steps in the argument for primeness in Theorem 2.1 (GLL), for negation-completeness in Theorems 2.1, 2.10, and 2.14, and implication-completeness in Theorems 2.1, 2.12, 2.17, and 2.18 and 2.6.2 all turn on the property that $B \notin \Gamma$ iff $\Gamma, B \vdash A$; here, where we have replaced $A$ by a class of formulas entailment, in conjunction with $\Gamma$, of any one of which suffices for nonmembership of $\Gamma$, we cannot make the moves we made in those earlier proofs. Our extension of GLHL does allow us to establish

TheOREM 12 If $C(\Sigma \cup\{A\}) \subseteq\{\wedge, \exists, \forall\}$ and $\Sigma \vdash_{\mathrm{CQL}} A$ then $\Sigma \vdash_{\mathrm{IQL}} A .^{30}$

As IQL has the subformula property and IQL and MQL do not differ as to the rules governing $\wedge, \exists$, and $\forall$, this can obviously be strengthened to read:

Theorem 13 If $C(\Sigma \cup\{A\}) \subseteq\{\wedge, \exists, \forall\}$ and $\Sigma \vdash_{\mathrm{CQL}} A$ then $\Sigma \vdash_{\mathrm{MQL}} A$.

3.6.2. The Rule $C D$ We add to IQL this strong form of $\vee$-elimination and its twin where the featured disjunction is $B \vee A$. We shall call both forms $C D$ :

\begin{tabular}{ccc} 
& {$[\forall v A(c / v)]$} & {$[B]$} \\
$A \vee B$ & $\vdots$ & $\vdots$ \\
\hline & $C$ & $C$ \\
\hline
\end{tabular}

subject to the restrictions that $c$ not occur in $B$ nor in any assumption on which $A \vee B$ depends.

Obviously we can derive the twin from the other using $\vee-I$ and $\vee-E$ but as we want to be careful about subformulas we shall adopt both forms.

We should note that this rule renders both $\vee$-E and $\forall$-I redundant, although not in such a way as to guarantee the entry of no new formulas. When no occurrences of $v$ in $A$ are free (more briefly, given present conventions: when $A$ is a formula) we get this imitation of $\vee-E::^{31}$

30 For a proof-theoretic proof of this result see Leblanc \& Thomason (1966, Theorem 5) (Leblanc, 1982, p. 402).

31 This relies on the admission of vacuous quantification. 


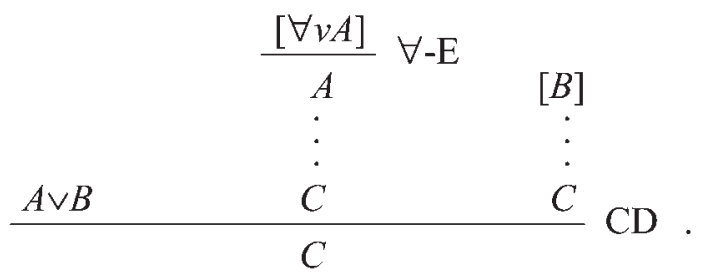

Mimicking $\forall$-I we have:

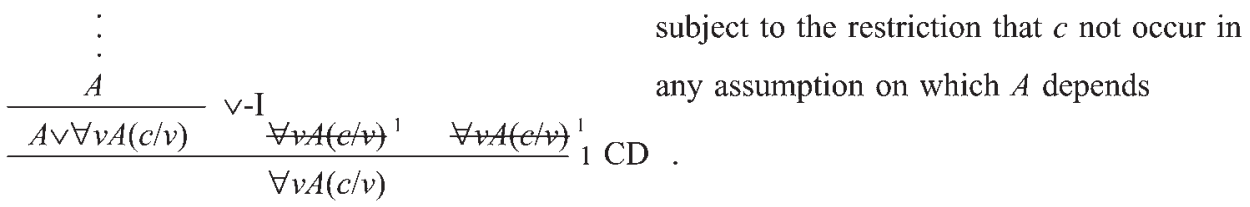

We can "liberalize" the rule $\mathrm{CD}$, lifting the ban on the featured constant's not occurring in both disjuncts. This requires a little care:

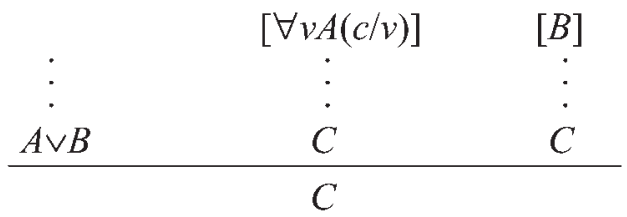

subject to the restrictions that $c$ not occur in any assumption on which $A \vee B$ depends, and if $B$ contains $c$ then $c$ does not occur in $C$ nor in any assumption other than $B$ in the subproof in which $B$ occurs as the assumption featured for discharge.

In the presence of $\exists$-I and $\exists$-E this is a derived rule, for, on the one hand, $A \vee B$ entails $A \vee \exists v B(c / v)$ by $\vee-\mathrm{I}, \vee-\mathrm{E}$, and $\exists-\mathrm{I}$, and, on the other, the restrictions imposed when $B$ contains $c$ are exactly such as to allow, through a single application of $\exists$-E, the transformation of the subproof in which $B$ occurs as an assumption into a subproof in which $\exists v B(c / v)$ occurs as assumption in its stead.

3.6.3. Kripke semantics for $I Q L+C D$ The logic IQL $+C D$ is known to be sound and complete with respect to Kripke models with constant domains. ${ }^{32}$

\subsubsection{The Generic Lindenbaum-Henkin Lemma: third extension}

THEOREM 14 Given $R$, when $\Sigma \nvdash_{R} A$ and where $c_{0}, c_{1}, \ldots, c_{n}, \ldots$, is an infinite sequence of constants all foreign to $\Sigma \cup\{A\}$ let $\Delta_{0}$ contain all subformulas of members of $\Sigma \cup\{A\}$ formed using $c_{0}, c_{1}, \ldots, c_{n}, \ldots$ and whatever other constants may occur in members of $\Delta_{0}$. Let $\Delta_{1}$ be the least set such that $A \in \Delta_{1}$ and $B \vee C(v / d) \in \Delta_{1}$ when $B \in \Delta_{1}, \forall v C \in \Delta_{0}$ and $d$ is a constant occurring in $\Delta_{0}$. Let $\Delta_{0} \cup \Delta_{1} \subseteq \Delta$, and let $R \backslash \Delta$ stand for the restriction of the rules in $R$ obtained by excluding rules governing those connectives and quantifiers that do not occur in any member of $\Delta$ and restricting those that remain to formulas in $\Delta$ and parametric instances of these. If the rules $\vee-I, \vee-E$, and

32 See, for example, Görnemann (1971) or Gabbay (1981, Lemmata 3-7, Corollary 8, pp. 50-53.) 
$C D$ either belong to $R$ or are safely $\Delta$-derived rules given the rules in $R$, then, keeping the numbering of clauses from previous sections,

(i) there is a well-behaved $\Delta_{0}, R \backslash \Delta$-theory $\Gamma$ such that $\Sigma \subseteq \Gamma \subseteq \Delta_{0}, A \notin \Gamma$;

(ii) $\Gamma$ is $\Delta_{0}$-prime;

(iii) if $\neg$ occurs in $\Delta_{0}$ and the rule of $\neg-E$ either belongs to $R$ or is a safely $\Delta$-derived rule given the rules in $R, \Gamma$ is $\Delta_{0}$-consistent;

(iv $v_{1}$ ) if $A=\neg D$, for some $D \in \Delta_{0}$ and the rule $\neg$-inv either belongs to $R$ or is a safely $\Delta$-derived rule given the rules in $R, \Gamma$ is $\Delta_{0}$-consistent;

$\left(v i_{1}\right)$ if an existential generalization belongs to $\Delta_{0}$ and the rule of $\exists$-E belongs to $R$ or is a safely $\Delta$-derived rule given the rules in $R$ then $\Gamma$ is $\Delta_{0}$-witness-complete;

(vi $\left.i_{2}\right) \Gamma$ is $\Delta_{0}$-counter-witness-complete.

Proof. Let $B_{0}, B_{1}, \ldots, B_{n}, \ldots$ be an enumeration of the formulas in $\Delta_{0}$, an enumeration indexed by $\mathbb{N}$. We define a sequence of extensions of $\Sigma$ and a sequence of $\Delta_{1}$-formulas $\left(\zeta_{n}\right)_{n \in \mathbb{N}}$ as follows:

(A) $\Gamma_{0}=\Sigma ; \zeta_{0}=A$;

for all $n \in \mathbb{N}$,

(B) $\Gamma_{n+1}=\Gamma_{n} \cup\left\{B_{n}\right\}, \zeta_{n+1}=\zeta_{n}$ if $B_{n}$ is not an existential generalization and $\Gamma_{n}, B_{n} \nvdash_{R \mid \Delta}$ $\zeta_{n}$,

$\Gamma_{n+1}=\Gamma_{n}, \zeta_{n+1}=\zeta_{n}$ if $\Gamma_{n}, B_{n} \vdash_{R \backslash \Delta} \zeta_{n}$, and $B_{n}$ is not a universal generalization, $\Gamma_{n+1}=\Gamma_{n} \cup\{\exists v D, D(v / c)\}$, where $D(v / c)$ is an instance employing a constant not occurring in $\Gamma_{n} \cup\left\{B_{n}, \zeta_{n}\right\}, \zeta_{n+1}=\zeta_{n}$ if $B_{n}$ is the formula $\exists v D$ and $\Gamma_{n}, B_{n} \nvdash_{R \backslash \Delta} \zeta_{n}$, $\Gamma_{n+1}=\Gamma_{n}, \zeta_{n+1}=\zeta_{n} \vee D(v / c)$, where $D(v / c)$ is an instance employing a constant not occurring in $\Gamma_{n} \cup\left\{B_{n}, \zeta_{n}\right\}$, if $B_{n}$ is the formula $\forall v D$ and $\Gamma_{n}, B_{n} \vdash_{R \mid \Delta} \zeta_{n}$.

We set $\Gamma=\cup_{n \in N} \Gamma_{n}$ and show, for all $n \in \mathbb{N}$, that $\Gamma_{n} \nvdash_{R \mid \Delta} \zeta_{n}$.

True by hypothesis for $n=0$. Induction hypothesis (IH): suppose that it's true for $n=k$. Cases:

(1) $\Gamma_{k+1}=\Gamma_{k} \cup\left\{B_{k}\right\}, \zeta_{k+1}=\zeta_{k}$ and $B_{k}$ is not an existential generalization: if $\Gamma_{k} \cup$ $\left\{B_{k}\right\} \vdash_{R \backslash \Delta} \zeta_{k}$ then, by construction, $\Gamma_{k+1}=\Gamma_{k}$ and $\Gamma_{k} \vdash_{R \backslash \Delta} \zeta_{k}$, contrary to IH;

(2) $\Gamma_{k+1}=\Gamma_{k}, \zeta_{k+1}=\zeta_{k}$ and $B_{k}$ is not a universal generalization: by $\mathrm{IH}, \Gamma_{k+1}=$ $\Gamma_{k} \nvdash_{R \mid \Delta} \zeta_{k}=\zeta_{k+1}$;

(3) $\Gamma_{k+1}=\Gamma_{k} \cup\{\exists v D, D(v / c)\}$, where $D(v / c)$ is an instance employing a constant not occurring in $\Gamma_{k} \cup\left\{B_{k}, \zeta_{k}\right\}, \zeta_{k+1}=\zeta_{k}$ and $B_{k}$ is the formula $\exists v D$ : much as in the proof of Theorem 11;

(4) $\Gamma_{k+1}=\Gamma_{k}, \zeta_{k+1}=\zeta_{k} \vee D(v / c)$, where $D(v / c)$ is an instance employing a constant not occurring in $\Gamma_{k} \cup\left\{B_{k}, \zeta_{k}\right\}$, and $B_{k}$ is the formula $\forall v D$ : suppose that $\Gamma_{k} \vdash_{R / \Delta}$ $\zeta_{k} \vee D(v / c)$; by IH, $\Gamma_{k} \nvdash_{R \backslash \Delta} \zeta_{k}$ so it must be the case that $\Gamma_{k}, \forall v D \vdash_{R \backslash \Delta} \zeta_{k}$; on the other hand, as $c$ is foreign to $\Gamma_{k} \cup\left\{\forall v D, \zeta_{k}\right\}$, we have, by an application of the rule $\mathrm{CD}$, that $\Gamma_{k} \vdash_{R \mid \Delta} \zeta_{k}$, contrary to IH.

By repeated appeals to $\vee$-I, $\zeta_{n} \vdash_{R \mid \Delta} \zeta_{n+k}, k>0$. Consequently, $\Gamma \nvdash_{R \mid \Delta} \zeta_{n}, n \in \mathbb{N}$, for if, on the contrary, $\Gamma \nvdash_{R \mid \Delta} \zeta_{k}$ for some $k$, then, proofs being finite, for some $m \in \mathbb{N}$, $\Gamma_{m} \vdash_{R \backslash \Delta} \zeta_{k}$; letting $n=\max \{k, m\}$, we contradict what has just been shown. If $\Gamma \vdash_{R \backslash \Delta} B$ then $\Gamma, B \nvdash_{R \backslash \Delta} \zeta_{n}$, for any $n \in \mathbb{N}$. In particular, then, as $B=B_{m}$, for some $m \in \mathbb{N}, \Gamma_{m}$, $B_{m} \nvdash \zeta_{m}$ and so $B=B_{m} \in \Gamma_{m+1} \subseteq \Gamma$. $\Gamma$ is a $\Delta_{0}, R\lceil\Delta$-theory. $B \notin \Gamma$ iff, for some $n \in \mathbb{N}$, $\Gamma, B \vdash_{R \mid \Delta} \zeta_{n}$, iff, for some $n \in \mathbb{N}, \Gamma, B \vdash_{R \backslash \Delta} \zeta_{n+k}, k \geq 0$. 
(ii) $\Gamma$ is $\Delta_{0}$-prime: if $B, C, B \vee C \in \Delta_{0}$ and if $B \notin \Gamma$ and $C \notin \Gamma$ then $\Gamma, B \vdash_{R \backslash \Delta} \zeta_{n}$ and $\Gamma, C \vdash_{R \backslash \Delta} \zeta_{m}$, hence, by $\vee$-I, if necessary, and $\vee$-E, $\Gamma, B \vee C \vdash_{R \mid \Delta} \zeta_{\max \{n, m\}}$, and so $B \vee C \notin \Gamma$.

(iii) and (iv 1 ) Proof is as in proof of Theorem 11.

(vi) $\Delta_{0}$-witness-completeness and $\Delta_{0}$-counter-witness-completeness are built into the construction (given the $\Delta_{0}, R \uparrow \Delta$-theoryhood of $\Gamma$ ).

Although an improvement over the second extension in that we have obtained $\Delta_{0^{-}}$ primeness of $\Gamma$, just as in 3.6.1 we cannot go on to obtain $\Delta_{0}$-negation- and $\Delta_{0^{-}}$ implication-completeness, for even when $A$ is of the form $\neg D$ the disjunctions $\zeta_{n}$ need not all be logically equivalent to formulas with negation dominant.

As a corollary to the $\Delta_{0}$-primeness guaranteed by the construction we have this parallel to Leblanc and Thomason's result in 3.6.1:

Theorem 15 If $C(\Sigma \cup\{A\}) \subseteq\{\wedge, \vee, \forall, \exists\}$ and $\Sigma \vdash_{\mathrm{CQL}} A$ then $\Sigma \vdash_{\mathrm{IQL}+\mathrm{CD}} A$.

3.6.5. $I Q L+C D$ does not have the subformula property $\forall x(F x \vee H a), \forall x(G x \vee$ $H a) \vdash \mathrm{IQL}+\mathrm{CD} \forall x(F x \wedge G x) \vee H a$. As the sequent is not intuitionistically valid, CD must be used. Subformulas are: $\forall x(F x \vee H a), \forall x(G x \vee H a), \forall x(F x \wedge G x) \vee H a, \forall x(F x \wedge G x), H a$, $F a \vee H a, G a \vee H a, F a \wedge G a, F a, G a, F b \vee H a, F b \vee H a, F b \wedge G b, F b, G b$, and so on for other constants. Applying CD to $F b \vee H a(G b \vee H a)$ requires $\forall x F x(\forall x G x)$, a nonsubformula, as assumption; applying CD to obtain $\forall x(F x \wedge G x) \vee H a$ requires applying it to an instance like $(F b \wedge G b) \vee H a$, which is not a subformula.

3.6.6. An unlikely formulation of CQL without redundant rules The rules are: $\wedge-\mathrm{I}$, $\wedge$-E, $\vee$-I, weak $\rightarrow$-I, Tarski's Rule, $\rightarrow$-E, Dilemma, $\neg$-E, $\forall$-E, $\exists$-I, $\exists$-E, CD. A derivation of $\Sigma \vdash A$ need contain only subformulas of members of $\Sigma \cup\{A\}$, disjunctions in the set $\cup_{n \in \mathbb{N}} \delta_{n}$ where $\delta_{0}=\{A\}$ and $\delta_{n+1}=\left\{B \vee D(v / c): B \in \delta_{n}, \forall v D\right.$ a subformula of a member of $\Sigma \cup\{A\}, c \in C\}$, with $C$ some countable set of constants foreign to $\Sigma \cup\{A\}$, and (vacuously quantified) universal generalizations of these (to make up for the absence of $\vee-E)$.

\subsection{A formulation of CQL with the (strict) subformula property.}

3.7.1. CQL with CD If we formulate CQL employing our favored version of CPL ( $\wedge-\mathrm{I}$, $\wedge$-E, $\vee$-I, $\vee$-E, weak $\rightarrow-$ I, Tarski's Rule, $\rightarrow$-E, Dilemma, $\neg$-E) and add, in addition to the standard quantifier rules $\forall-\mathrm{I}, \forall-\mathrm{E}, \exists-\mathrm{I}$, and $\exists$-E, the rule $\mathrm{CD}$ then minor modifications to 3.6.4's third extension of GLHL will deliver:

THEOREM 16 Let RCCDQ be the just specified set of rules. When $\Sigma \nvdash_{R C C D Q} A$ and where $c_{0}, c_{1}, \ldots, c_{n}, \ldots$, is an infinite sequence of constants all foreign to $\Sigma \cup\{A\}$ let $\Delta_{0}$ contain all subformulas of members of $\Sigma \cup\{A\}$ formed using $c_{0}, c_{1}, \ldots, c_{n}, \ldots$ and whatever other constants may occur in members of $\Delta_{0}$. Let $\Delta_{1}$ be the least set such that $A \in \Delta_{1}$ and $B \vee C(v / d) \in \Delta_{1}$ when $B \in \Delta_{1}, \forall v C \in \Delta_{0}$ and $d$ is a constant occurring in $\Delta_{0}$. Let $\Delta_{0} \cup \Delta_{1} \subseteq \Delta$, and let $R \nabla \Delta$ stand for the restriction of the rules in RCCDQ obtained by excluding rules governing those connectives and quantifiers that do not occur in any member of $\Delta$ and restricting those that remain to formulas in $\Delta$ and parametric instances of these. Then, keeping the numbering of clauses from previous sections,

(i) there is a well-behaved $\Delta_{0}, R \backslash \Delta$-theory $\Gamma$ such that $\Sigma \subseteq \Gamma \subseteq \Delta_{0}, A \notin \Gamma$;

(ii) $\Gamma$ is $\Delta_{0}$-prime;

(iii) $\Gamma$ is $\Delta_{0}$-consistent; 
(iv $\left.v_{\mathrm{Di}}\right) \Gamma$ is $\Delta_{0}$-negation-complete;

$\left(v_{\mathrm{T}}{ }^{\prime} \mathrm{ski}\right) \Gamma$ is $\Delta_{0}$-implication-complete;

(vi $\left.i_{1}\right) \Gamma$ is $\Delta_{0}$-witness-complete;

(vi $\left.i_{2}\right) \Gamma$ is $\Delta_{0}$-counter-witness-complete.

Proof. Theorem 14 takes care of all except (iv $\left.\mathrm{Di}_{\mathrm{Di}}\right)$ and ( $\left(\mathrm{v}_{\mathrm{T}}\right.$ 'ski $)$.

(iv $\left.{ }_{\mathrm{Di}}\right) \Gamma$ is $\Delta_{0}$-negation-complete: if $B, \neg B \in \Delta_{0}$ and if $B \notin \Gamma$ and $\neg B \notin \Gamma$ then $\Gamma$, $B \vdash_{R \mid \Delta} \zeta_{n}$ and $\Gamma, \neg B \vdash_{R \mid \Delta} \zeta_{m}$, hence, by $\vee$-I if necessary, $\Gamma, B \vdash_{R \mid \Delta} \zeta_{\max \{n, m\}}$ and $\Gamma$, $\neg B \vdash_{R \mid \Delta} \zeta_{\max \{n, m\}}$, whence, by Dilemma, $\Gamma \vdash_{R \backslash \Delta} \zeta_{\max \{n, m\}}$, which we know not to be the case.

$\left(\mathrm{v}_{\mathrm{T}}{ }^{\prime} \mathrm{ski}\right) \Gamma$ is $\Delta_{0}$-implication-complete: proved analogously with Tarski's Rule for Dilemma.

Furthermore, from careful inspection of the proof of Theorem 14 we learn that when $\Sigma$ $\vdash_{R C C D Q} A$

(1) the only nonsubformulas that need occur in a proof in this version of CQL belong to a sequence $A, A \vee D_{1}\left(v_{1} / c_{1}\right),\left(A \vee D_{1}\left(v_{1} / c_{1}\right)\right) \vee D_{2}\left(v_{2} / c_{2}\right), \ldots(\ldots)(A \vee$ $\left.\left.D_{1}\left(v_{1} / c_{1}\right)\right) \vee \ldots\right) \vee D_{n}\left(v_{n} / c_{n}\right)$ where each of the $D_{i}\left(v_{i} / c_{i}\right)$ 's is an instance of a universal generalization $\forall v_{i} D_{i}$ that is a subformula of some member of $\Sigma \cup\{A\}$ and $c_{i+1}$ is foreign to $\Sigma \cup\left\{A, A \vee D_{1}\left(v_{1} / c_{1}\right),\left(A \vee D_{1}\left(v_{1} / c_{1}\right)\right) \vee D_{2}\left(v_{2} / c_{2}\right), \ldots(\ldots(A \vee\right.$ $\left.\left.\left.D_{1}\left(v_{1} / c_{1}\right)\right) \vee \ldots\right) \vee D_{i}\left(v_{i} / c_{i}\right)\right\}$ (although, obviously, not all members of the sequence are nonsubformulas);

(2) as the sequence contains the only nonsubformulas (if any), the longest nonsubformula must enter in an application of $\mathrm{V}-\mathrm{I}$;

(3) that once entered such nonsubformula can only occur as $(a)$ the premise to an application of $\mathrm{V}-\mathrm{I},(b)$ as the "major premise", that is, as the disjunction to which the rule is applied, to an application of the rule $\mathrm{CD}$, or $(c)$ as the conclusion of the subproof in an application of $\exists$-E or as the conclusion of both subproofs in an application of $\vee-E$, Dilemma, Tarski's Rule, or the rule CD, whose conclusion must itself be an instance of that same formula;

(4) that the conclusion of an application of CD eliminating a member of the sequence is the previous member of the sequence;

(5) that in the application of CD eliminating $\left(\ldots\left(A \vee D_{1}\left(v_{1} / c_{1}\right)\right) \vee \ldots\right) \vee D_{i}\left(v_{i} / c_{i}\right)$, in the subproof in which $\left(\ldots\left(A \vee D_{1}\left(v_{1} / c_{1}\right)\right) \vee \ldots\right)$ is derived from $\forall v_{i} D_{i}$ no undischarged assumption contains the constant $c_{i}$.

\subsubsection{The lie of the land}

3.7.2.1. Quantifying within the scope of a disjunction A naïve attempt, the most naïve, to derive $\forall x F x \vee G a$ from $\forall x(F x \vee G a)$ without the CD rule fails very straightforwardly because of the restrictions placed on $\forall$-I. It looks like this:

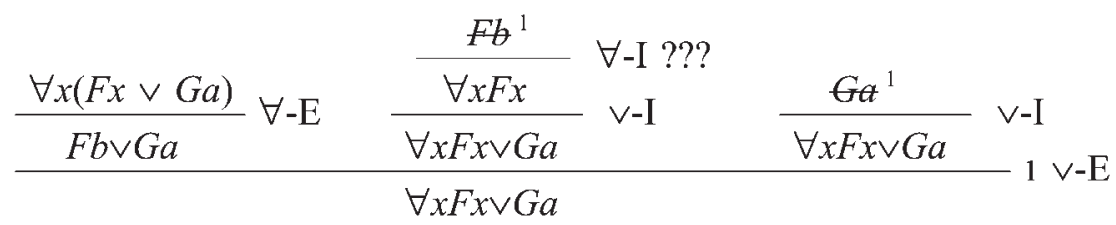


It fails exactly because we are precluded from applying $\forall$-I at the point marked '???' in the subproof as $b$ occurs in the assumption $F b$.

Naïvely, we should ask, what exactly is wrong with this application of $\forall$-I? Certainly it breaches the standard restrictions but these are motivated by the need to have the name generalized upon stand, in some sense, for "the general case", not a particular named case, for we certainly do not want to be entitled to infer $\forall x F x$ from $F b$ in all circumstances, but here, plausibly given its provenance, standing for the general case is exactly what the name $b$ is doing. The guiding thought in what follows is that we ought to be allowed to generalize in this sort of case. We must, then, weaken the restrictions on $\forall-\mathrm{I}$-and specify what "this sort of case" amounts to.

If we have names $b_{0}, b_{1}, \ldots, b_{n}, \ldots$ for everything, $\forall x(F x \vee G a)$ amounts (loosely!) to $\left(F b_{0} \vee G a\right) \wedge\left(F b_{1} \vee G a\right) \wedge \ldots \wedge\left(F b_{n} \vee G a\right) \wedge \ldots$ from which, extrapolating from the finite case, we should feel justified in inferring $\left(F b_{0} \wedge F b_{1} \wedge \ldots \wedge F b_{n} \wedge\right.$ ...) $\vee G a$, which amounts to $\forall x F x \vee G a$. So, when we have names for everything, or, rather, when it makes sense to think that we could have names for everything, it does make sense to think of $F b$ as standing for the general case and universally generalizing on it in the "proof" above. When does it make sense to think that we could have names for everything?-When we are dealing with a fixed domain. On the other hand, in the Kripke semantics for IQL the domain may grow, and when growth occurs talk of having a name for everything makes little sense for when there is growth we must end up having either nondenoting names at some nodes or unnamed things at others.

The standard natural deduction formulations of IQL and CQL do not differ as to the restrictions placed on application of $\forall$-I. What the last three paragraphs aim to motivate is the thought that in the classical setting those restrictions are unduly tight. What "this sort of case" amounts to is having a fixed domain, and so it makes sense to suppose that there could be names for everything in that domain. With that in mind, we want to be able to derive all the following:

$$
\begin{aligned}
& \forall x(F x \vee H a) \vdash \forall x F x \vee H a ; \forall x(F x \vee H a), \forall x(G x \vee H a) \vdash \forall x(F x \wedge G x) \vee H a ; \forall x \forall y \\
& (F x \vee G y) \vdash \forall x F x \vee \forall y G y ; \forall x(F x \vee G x) \vdash \forall x F x \vee \exists x G x .
\end{aligned}
$$

On the other hand, we do not want to be able to derive:

$$
\forall x(F x \vee G x) \vdash \forall x F x \vee \forall x G x ; \forall x \forall y(R x y \vee R y x) \vdash \exists x \forall y R x y \vee \exists x \forall y R y x
$$

Finally, we note the "existential reading" given one of the disjuncts in the liberalized version of the rule $\mathrm{CD}$ at the end of 3.6.2.

\subsubsection{Raising and eliminating applications of $C D$ to nonsubformulas Let $E \vee D(v / c)$} be the longest nonsubformula in an $R C C D Q$ derivation of $A$ from $\Sigma$. It enters any branch of the proof in which it occurs by $\vee$-I. Immediately below it stand zero or more further occurrences as conclusions of applications of $\exists-E, \vee-E$, Dilemma, Tarski's Rule, or CD, until it is eliminated by an application of CD. Let the depth of an application of CD in which $E \vee D(v / c)$ is eliminated be the largest number of contiguous occurrences of that very formula standing above it.

If the depth is zero $E \vee D(v / c)$ occurs in a proof segment of one of these forms: 

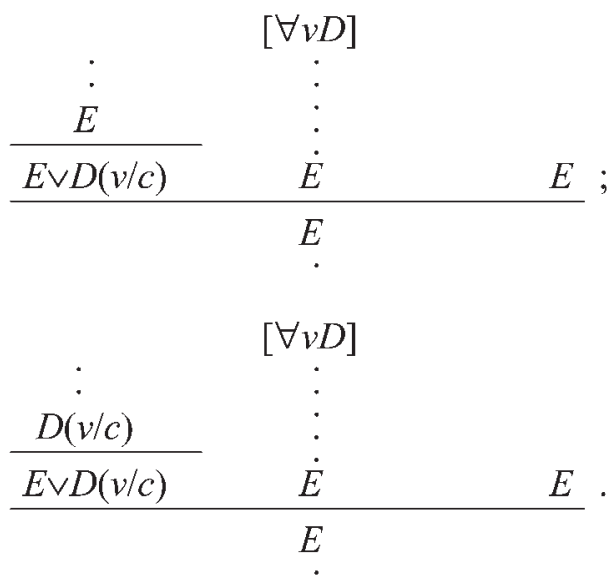

The first involves an obviously eliminable detour. The second too involves a pointless detour given that, in its standard setting, in an application of $\mathrm{CD} c$ does not occur in any assumption on which $E \vee D(c / v)$ depends.

In the case of occurrences at greater depth we have something that looks more like this:

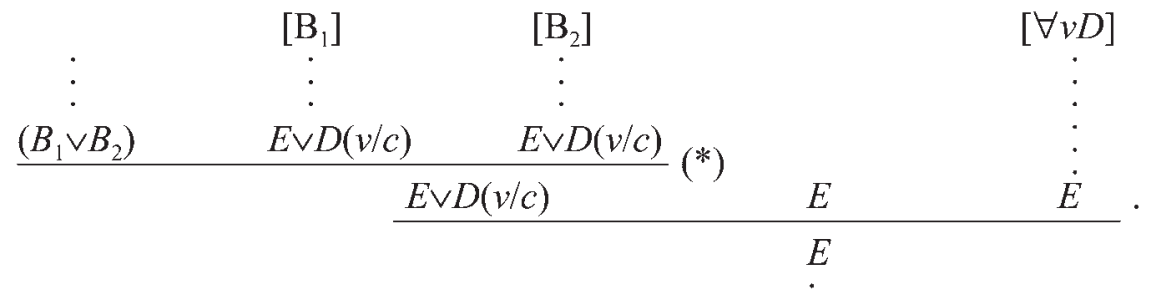

where at $(*)$ we have an application of one of $\vee$-E, Dilemma, Tarski's Rule, or CD. In first and last cases there is a major premise $B_{1} \vee B_{2}$; in the last case one of the disjuncts occurs universally generalized as an assumption in the subproof in which it occurs as minor premise, that is, as an assumption to be discharged in the application of the rule.

It would be nice if we could reduce the depth at which the application of CD eliminates $E \vee D(v / c)$ by performing two applications of CD in the subproofs before the application of whichever rule it is at $\left(^{*}\right)$. That is, we would like to be able to transform the above into:

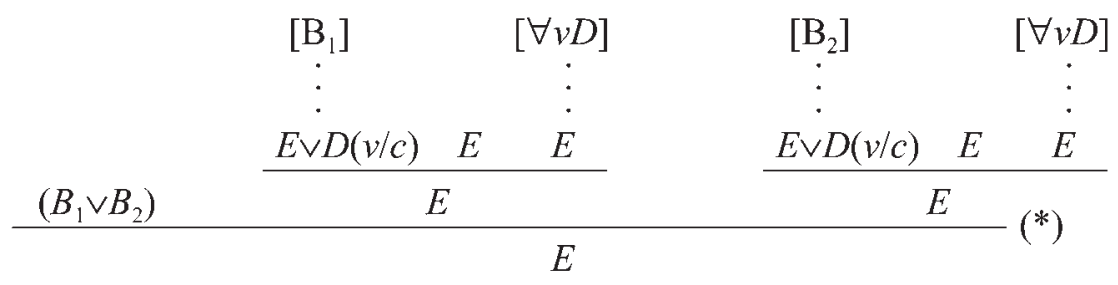

Unfortunately, we have no guarantee that the constant $c$ does not occur in one or both of $B_{1}$ and $B_{2}$, so we have no guarantee that such applications would meet the letter of the rule $\mathrm{CD}$. 
3.7.2.3. Mimicking the classical sequent calculus The sequent calculus formulation of classical first-order logic has the subformula property but allows a multiplicity of formulas in the succeedent. On the standard reading, at least one of these is true if all formulas in the antecedent are true. Consequently the disjunction of all formulas in the succeedent is true when all formulas in the antecedent are true. Thinking in terms of this disjunction, what the classical sequent calculus allows is quantification on a disjunct subordinate to an occurrence of $\vee$. For example, we have:

Sequent calculus derivation of $\forall x(F x \vee G a) \vdash \forall x F x \vee G a$

$$
\begin{aligned}
& F b \vdash F b \quad G a \vdash G a \\
& F b \vdash F b, G a \quad G a \vdash F b, G a \\
& \frac{F b \vee G a \vdash F b, G a}{\forall x(F x \vee G a) \vdash F b, G a} \\
& \forall x(F x \vee G a)+\forall x F x, G a \\
& \forall x(F x \vee G a) \vdash \forall x F x \vee G a
\end{aligned}
$$

Single conclusion rewrite

$\frac{\frac{F b \vdash F b}{F b \vdash F b \vee G a} \frac{G a \vdash G a}{G a+F b \vee G a}}{\frac{F b \vee G a \vdash F b \vee G a}{\forall x(F x \vee G a)+F b \vee G a}} \frac{\frac{\forall x(F x \vee G a)+\forall x F x \vee G a}{\forall x(F x \vee G a)+\forall x F x \vee G a}}{}$

If we are to emulate the simplicity of application of the rules in the left-hand derivation in a single-conclusion system, thereby avoiding introduction of a quantifier into a position subordinate to $\vee$, we must allow the universal generalization $\forall x F x$ to enter "as soon as possible", that is, right at the top of the right-hand derivation, for that is the only opportunity we have to introduce the universal quantifier not subordinate to an occurrence of disjunction. We must then ensure that, as $F b \vdash \forall x F x$ is not generally valid, we constrain its possible occurrences so that by the point at which we have completed application of $\vee-E$ (or Dilemma or Tarski's Rule), validity is not compromised.

3.7.2.4. Peculiar practices The techniques we have used above fail in the presence of the rule $\mathrm{CD}$. An indication of this is provided by the details of the completeness proofs for IQL + CD obtained by Görnemann (1971) and Gabbay (1981). Another is provided by the following example. The classically valid, intuitionistically invalid $\exists x(\exists x \neg F x \rightarrow \neg F x)$ is derivable in $\mathrm{KQL}+\mathrm{CD}$. (Exercise for the reader.) Its negation-free analogue $\exists x(\exists x F x \rightarrow$ $F x$ ) is not derivable in the stronger $\mathrm{LQL}+\mathrm{CD}$. However, models showing this have an unexpected property. LQL $+\mathrm{CD}$ is sound (and, I assume, complete) with respect to Kripke models for IQL in which the nodes are linearly ordered and every node has the same domain. $\exists x(\exists x F x \rightarrow F x)$ can only fail to be verified at the base node if there is an infinite descending $\preceq$-chain of distinct nodes above the base node at each of which $\exists x F x$ is verified, for there must be no first node (with respect to the ordering $\preceq$ ) at which $\exists x F x$ is verified. The standard methods of construction, which proceed by the addition of formulas, will not produce such a sequence: here we seem to be concerned rather with the subtraction of formulas.

\subsubsection{The rule $\forall-I^{\prime}$}

3.7.3.1. Flagging Call the assumptions occurring undischarged in the subproofs and discharged in applications of $\vee$-E, Dilemma, and Tarski's Rule the minor premises to the application of the rule. In the case of $\vee-E$, call the eliminated disjunction the major premise. One may flag one or both of the minor premises to an application of $\vee$-E, Dilemma, or 
Tarski's Rule by a constant occurring in it. In the case of $\mathrm{V}$-E this is subject to the proviso that no assumption on which the major premise depends and which contains that constant is not flagged by that constant.

\subsubsection{Weakening the restrictions on $\forall-I$ The rule $\forall-\mathrm{I}^{\prime}$ :}

One may infer $\forall v D(c / v)$ from $D$ provided $c$ flags each occurrence of any assumption containing $c$ on which $D$ depends.

3.7.4. CQL reformulated The propositional component is as before, save for restrictions to be entered immediately below: $\wedge$-I, $\wedge$-E, $\vee-\mathrm{I}, \vee$-E, weak $\rightarrow-$-I, Tarski's Rule, $\rightarrow-\mathrm{E}$, Dilemma, $\neg$-E. To this we add $\forall-\mathrm{I}^{\prime}, \forall$-E, $\exists$-I , and $\exists$-E. We shall call this CQL'.

In single flagging, that is, in flagging only one of the minor premises, we are reading minor premises containing $a$ of, say, an application of Dilemma- $\phi(v / a)$ and $\neg \phi(v / a)-$ as in effect being either the pair $\forall v \phi$ and $\exists v \neg \phi$ or the pair $\exists v \phi$ and $\forall v \neg \phi$. This is legitimated by two facts: from assumptions $\phi(v / a)$ and $\neg \phi(v / a)$ we can use, firstly, $\vee$-I to get $\phi(v / a) \vee \neg \phi(v / a)$ in each subproof, and hence, by Dilemma, $\phi(v / a) \vee \neg \phi(v / a)$ no longer dependent on those assumptions; from there, classically, we can get to each of $\forall v \phi \vee \exists v \neg \phi$ and $\exists v \phi \vee \forall v \neg \phi$ in which the disjuncts may serve as minor premises to an application of $\checkmark$-E. In effect single flagging collapses those several steps to one. With that in mind, we note a limitation that must be observed when several names occur flagged. Consecutive applications of CD allow us to pass from $\forall x \forall y(R x y \vee \neg R x y)$ first to $\forall x(\forall y R x y \vee \exists y \neg R x y)$ then to $\exists x \forall y R x y \vee \forall x \exists y \neg R x y$, but we cannot go on to reach $\exists x \forall y R x y \vee \exists y \forall x \neg R x y$ (and rightly so). In allowing separate treatment of the disjuncts, we must add a restriction that has this limitative effect:

Restrictions under single flagging If in an application of $\vee$-E, Dilemma, or Tarski's Rule there are constants common to both minor premises and both minor premises are flagged by some but different ones among these common constants then, if there are applications in both of the subproofs of both $\forall-\mathrm{I}^{\prime}$ and $\exists-\mathrm{I}$ in which these common constants are generalized upon, the order in which these constants are generalized upon in both subproofs must be the same to within permutations for generalizations employing the same quantifier.

If in an application of $\mathrm{V}$-E, Dilemma, or Tarski's Rule a constant flagging one of the minor premises is generalized upon using $\forall-\mathrm{I}^{\prime}$, then that constant must not occur in the conclusion of the application of the rule, nor may it occur in any assumption other than the other minor premise in the other subproof.

Examples may make the intention clearer. The proof immediately below goes through; the annotation indicates the sense in which the order of generalization is the same (to within permutations with the same quantifier).

\begin{tabular}{|c|c|}
\hline Rabed ${ }^{c d 1}$ & $\Rightarrow$ Rabed ${ }^{a b} 1$ \\
\hline$\forall w R a b c w \quad[\forall d]$ & $\exists z \cdot \operatorname{Rabzd}[\exists c]$ \\
\hline$\forall z \forall w R a b z w \quad[\forall c]$ & $\exists w \exists z \neg R a b z d \quad[\exists d]$ \\
\hline$\exists y \forall z \forall w R a y z w$ & $\forall x \exists w \exists z \neg R x b z w \quad[\forall b]$ \\
\hline$\exists x \exists y \forall z \forall w R x y z w \quad[\exists b]$ & $\forall y \forall x \exists w \exists \exists \neg R x y z w \quad[\forall a]$ \\
\hline$\exists x \exists y \forall z \forall w R x y z w \vee \forall y \forall x \exists w \exists z \neg R x y w z$ & $\exists x \exists y \forall z \forall w R x y z w \vee \forall y \forall x \exists w \exists z \neg R x y w z$ \\
\hline
\end{tabular}

The next "proof" rightly fails to go through; what blocks it is exactly the order-tracking restriction. 


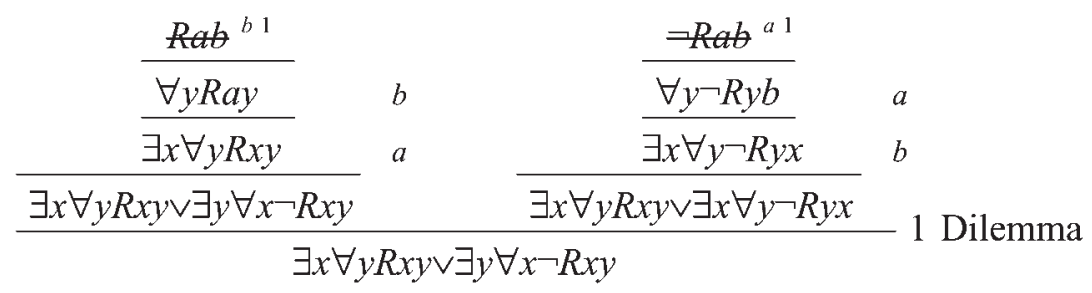

Without the second restriction, this slightly devious "proof" would be acceptable (which clearly it ought not to be):

$$
\frac{\frac{\frac{F e^{c 1}}{\forall x F x} \forall-\mathrm{I}^{\prime}}{F c \rightarrow \forall x F x} \text { weak } \rightarrow-\mathrm{I} \quad F c \rightarrow \forall x F x^{1}}{\frac{F c \rightarrow \forall x F x}{\forall x(F x \rightarrow \forall x F x)} \forall-\mathrm{I}} 1 \text { Tarski's Rule ??? }
$$

At first sight double flagging may look as though, with minor premises $\phi(v / a)$ and $\neg \phi(v / a)$ containing $a$ in an application of Dilemma, we are in effect reading these as $\forall v \phi$ and $\forall v \neg \phi$. In fact the inspiration is quite other, and indeed less convoluted than with single flagging. The aim is to have the pair of disjuncts, $\phi(v / a)$ and $\neg \phi(v / a)$, do duty for the universally generalized disjunction $\forall v(\phi \vee \neg \phi)$. Thus if in each of the subproofs, one headed by $\phi(v / a)$, the other by $\neg \phi(v / a)$ we obtain the same formula containing $a$, we want, provided no other assumptions contain specific information about the object named by $a$, to say that that conclusion holds generally. With that in mind we introduce this restriction on applications of $\vee$-E, Dilemma, and Tarski's Rule:

Restriction on double flagging If, in an application of $\vee-E$, Dilemma, or Tarski's Rule there are constants common to both minor premises and both minor premises are flagged by a constant $c$ common to both, then in the subproofs headed by these minor premises $\forall-\mathrm{I}^{\prime}$ must be applied to the same formula containing $c$ in both subproofs. This restriction blocks this attempt to derive $\forall x F x \vee \forall x G x$ from $\forall x(F x \vee G x)$ :

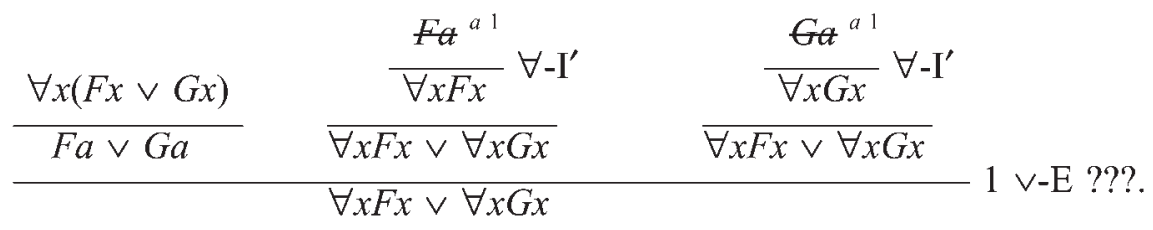

Notice that it is the putative application of $\mathrm{V}-\mathrm{E}$ that is incorrect; the two applications of $\forall-\mathrm{I}^{\prime}$ are perfectly fine.

3.7.4.1. A remark on the soundness of $C Q L^{\prime} \quad$ With the explanations just given in mind, it perhaps needs little argument that $\mathrm{CQL}^{\prime}$ is sound. Any doubt must focus on uses of $\forall-\mathrm{I}^{\prime}$ that are not also uses of orthodox $\forall$-I and the restrictions just introduced on applications of $\vee$-E, Dilemma, and Tarski's Rule. In any application of $\forall-\mathrm{I}^{\prime}$ all assumptions containing the name generalized upon must be flagged by that name. In single flagging this means that the assumption is doing duty for the formula obtained by universally generalizing on that name. If double flagging is involved, the minor premises stand in for the universal 
generalization of their disjunction. Now, in the case of applications of $\mathrm{V}$-E one may have to track back some way through a proof to locate the part of the derivation which licenses the flagging, that is, licenses the general reading but there must be some such point where flagging of the name in question first appears and there we are dealing with either a disjunction to which $\forall$-I is applicable in virtue of its containing the name in question but depending on no assumptions containing that name or on minor premises in applications of Dilemma and Tarski's Rule the universal generalization of the disjunction of which can legitimately be inferred.

The soundness of applications of $\forall-\mathrm{I}^{\prime}$ then follows from the classical soundness of the inferences $\forall v(\phi v \vee \psi) \vdash \forall v \phi v \vee \psi, \forall v(\phi v \vee \psi v) \vdash \forall v \phi v \vee \exists v \psi$, and $\forall v(\phi v \vee \psi v) \vdash \exists v \phi v \vee$ $\forall v \psi$, and, under double flagging, the (intuitionist) validity of $\forall v(\phi v \vee \psi v), \forall v(\phi v \rightarrow \chi v)$, $\forall v(\psi v \rightarrow \chi v) \vdash \forall v \chi v$.

The first restriction under single flagging on $\vee$-E, Dilemma, and Tarski's Rule is needed to ensure consistency of the $\forall / \exists$ disjunction reading with multiple quantifiers. Starting from $\forall v \forall u(\phi(v, u) \vee \psi(v, u))$ we can "push" one or other of the quantifiers "through the brackets" and, indeed, both but in the case of both they should be thought of being pushed through one at a time, to get, say, first $\forall u(\forall v \phi(v, u) \vee \exists v \psi(v, u))$ then $\exists u \forall v \phi(v, u) \vee$ $\forall u \exists v \psi(v, u)$.

The second restriction under single flagging ensures that the unflagged minor premise is treated as equivalent to the existential generalization obtained by generalizing the occurrences of the flagging constant in it.

3.7.4.2. $C Q L^{\prime}$ has the subformula property We transform $R C C D Q$ proofs. Recall from 3.7.1 that when $\Sigma \vdash_{R C C D Q} A$

(1) the only nonsubformulas that need occur in a proof in this version of CQL belong to a sequence $A, A \vee D_{1}\left(v_{1} / c_{1}\right),\left(A \vee D_{1}\left(v_{1} / c_{1}\right)\right) \vee D_{2}\left(v_{2} / c_{2}\right), \ldots(\ldots)(A \vee$ $\left.\left.D_{1}\left(v_{1} / c_{1}\right)\right) \vee \ldots\right) \vee D_{n}\left(v_{n} / c_{n}\right)$ where each of the $D_{i}\left(v_{i} / c_{i}\right)$ 's is an instance of a universal generalization $\forall v_{i} D_{i}$ that is a subformula of some member of $\Sigma \cup\{A\}$ and $c_{i+1}$ is foreign to $\Sigma \cup\left\{A, A \vee D_{1}\left(v_{1} / c_{1}\right),\left(A \vee D_{1}\left(v_{1} / c_{1}\right)\right) \vee D_{2}\left(v_{2} / c_{2}\right), \ldots(\ldots(A \vee\right.$ $\left.\left.\left.D_{1}\left(v_{1} / c_{1}\right)\right) \vee \ldots\right) \vee D_{i}\left(v_{i} / c_{i}\right)\right\}$ (although, obviously, not all members of the sequence are nonsubformulas);

(2) as the sequence contains the only nonsubformulas (if any), the longest nonsubformula must enter in an application of $\mathrm{V}-\mathrm{I}$;

(3) that once entered such nonsubformula can only occur as $(a)$ the premise to an application of $\mathrm{V}-\mathrm{I},(b)$ as the "major premise", that is, as the disjunction to which the rule is applied, to an application of the rule $\mathrm{CD}$, or $(c)$ as the conclusion of the subproof in an application of $\exists$-E or as the conclusion of both subproofs in an application of $\vee$-E, Dilemma, Tarski's Rule, or the rule CD, whose conclusion must itself be an instance of that same formula;

(4) that the conclusion of an application of CD eliminating a member of the sequence is the previous member of the sequence;

(5) that in the application of CD eliminating $\left(\ldots\left(A \vee D_{1}\left(v_{1} / c_{1}\right)\right) \vee \ldots\right) \vee D_{i}\left(v_{i} / c_{i}\right)$, in the subproof in which $\left(\ldots\left(A \vee D_{1}\left(v_{1} / c_{1}\right)\right) \vee \ldots\right)$ is derived from $\forall v_{i} D_{i}$ no undischarged assumption contains the constant $c_{i}$.

Consider the last occurrence of the longest nonsubformula, say $E \vee D(v / c)$. It stands as major premise in an application of CD containing a subproof comprising $E$ alone and a subproof with $\forall v D$ as assumption and $E$ as conclusion. By construction, $c$ need occur in 
no formulas below the last occurrence of $E \vee D(v / c)$ and its last occurrence depends on no assumption in which $c$ occurs unflagged. We know that this last occurrence of $E \vee D(v / c)$ can be done away with if it is of depth zero (3.7.2.2). We show that if the depth is greater than zero it can be reduced through uses of $\forall-\mathrm{I}^{\prime}$.

(i) If the topmost occurrence of $E \vee D(v / c)$ in the sequence of contiguous occurrences above the last occurs in a subproof of $\exists$-E, then introduction of $E \vee D(v / c)$ (by $\vee-I$ ) can be deferred until after the application of $\exists$-E: the application of $\exists$-E then yields either $E$ or $D(v / c)$ as conclusion.

(ii) If the topmost occurrence occurs as conclusion to both subproofs of $\vee$-E, Dilemma, Tarski's Rule, or CD then, being introduced in both subproofs by $\vee-I$, if both applications obtain it from the same disjunct, application of $\vee-I$ can be deferred until after application of the rule in question.

(iii) If the topmost occurrence occurs as conclusion to both subproofs of $\vee$-E, Dilemma, Tarski's Rule, or CD and in one subproof is obtained by $\vee$-I from $E$ and in the other from $D(v / c)$, then drop the application of $\vee$-I to $E$ and, in the other subproof, flag all assumptions containing $c$ upon which the occurrence of $D(v / c)$ depends with $c$, apply $\forall-\mathrm{I}^{\prime}$ to obtain $\forall v D$, and introduce beneath it the subproof of $E$ with $\forall v D$ as assumption from the application of CD that eliminates $E \vee D(v / c)$. (This may require duplication of subproofs, depending on the number of occurrences of $\forall v D$ as an undischarged assumption in that subproof.) Now apply the deferred rule $-\vee-E$, Dilemma, Tarski's Rule, or CD—to obtain $E$ as interim conclusion; introduce $E \vee D(v / c)$ using $\vee$-I.

In carrying out this step we find that our restrictions on flagging are just flexible enough to allow the reduction in depth that we are looking for. Although the instruction above may look as though it only gives rise to single flagging, it may be that we find that we have flagged both minor premises in an application of $\vee$-E, Dilemma, Tarski's Rule, or CD with the same constant. However, whenever that happens, we can proceed in compliance with the restriction under double flagging. This sort of case arises when we have two applications of $\vee-E$, Dilemma, Tarski's Rule, or CD occurring in the subproofs of a further application of one of these rules. With Dilemma, if one minor premise contains the constant $c$ the other must; with $\vee$-E, Tarski's Rule, and CD, the constant $c$ may occur in both minor premises. Without loss of generality we may consider a case like this:

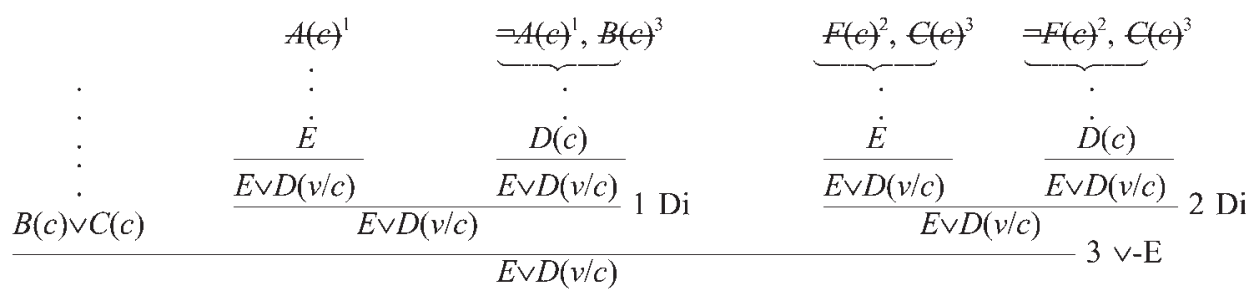

Following the instructions above and noting that $c$ occurs in both disjuncts, we obtain this proof. 


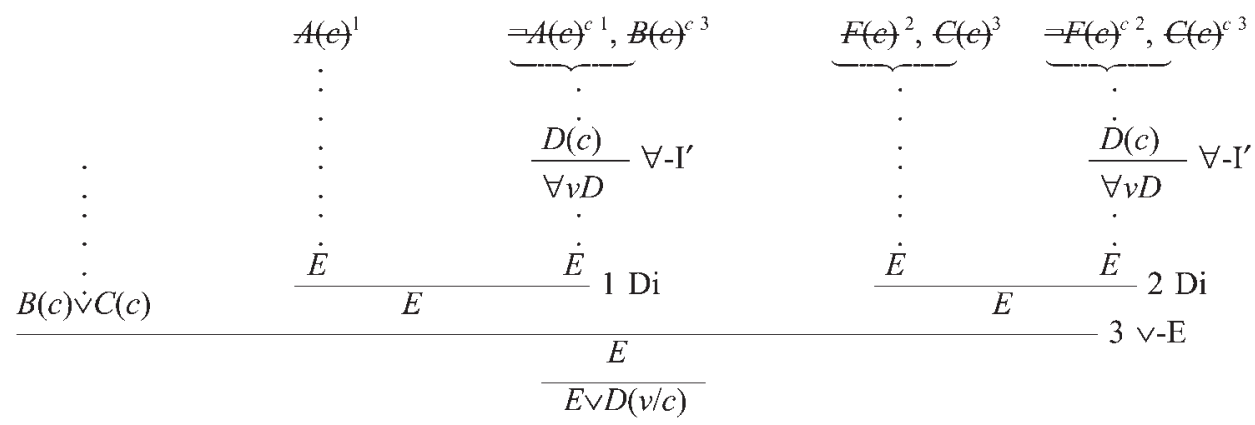

Because $\forall-\mathrm{I}^{\prime}$ is applied to the same formula containing $c$ in both subproofs the restriction under double flagging is met.

By repeated application of Steps (i), (ii), and (iii), the depth of occurrence of $E \vee D(v / c)$ can be reduced to zero, at which point the last occurrence may itself be eliminated. The procedure is then applied to the remaining occurrences (if any) of $E \vee D(v / c)$, working up from the bottom. When all occurrences of the longest nonsubformula have been eliminated, we turn to its predecessor in the sequence of nonsubformulas (which is $E$ if $E$ is not a subformula).

Lastly, applications of CD to subformulas are rewritten as follows. Where we have

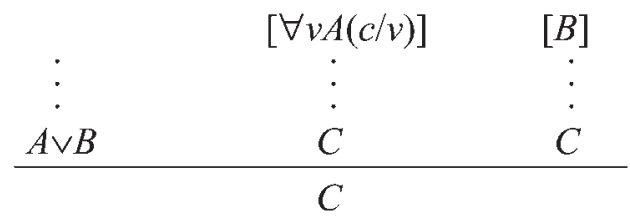

subject to the restrictions that $c$ not occur in any assumption on which $A \vee B$ depends, and if $B$ contains $c$ then $c$ does not occur in $C$ nor in any assumption other than $B$ in the subproof in which $B$ occurs as the assumption featured for discharge, we replace it with

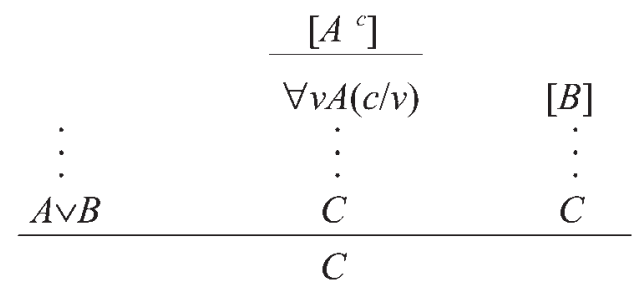

This completes the proof that the system $\mathrm{CQL}^{\prime}$ is a formulation of classical first-order logic with the subformula property.

§4. Acknowledgment. The author thanks Tor Sandqvist, referees, and audiences at the University of St Andrews for helpful comments.

\section{BIBLIOGRAPHY}

Beth, E. W., \& Leblanc, H. (1960). A note on the intuitionist and the classical propositional calculus. Logique et Analyse, 11-12, 174-176. Reprinted in Existence, Truth, and Provability. Albany, NY: State University of New York Press, pp. 382-384. 
Boolos, G. (1984). Trees and finite satisfiability: Proof of a conjecture of Burgess. Notre Dame Journal of Formal Logic, 25, 193-197.

Curry, H. B. (1963). Foundations of Mathematical Logic. New York: McGraw Hill. Reprinted with corrections, New York: Dover Publications, 1977.

Dummett, M. (1991). The Logical Basis of Metaphysics. London: Duckworth.

Fitting, M. (1969). Intuitionist Logic, Model Theory and Forcing. Amsterdam, The Netherlands: North-Holland.

Gabbay, D. (1981). Semantical Investigations in Heyting's Intuitionist Logic. Dordrecht, The Netherlands: Reidel.

Görnemann, S. (1971). A logic stronger than intuitionism. Journal of Symbolic Logic, 36(2), 249-261.

Horn, A. (1962). The separation theorem of intuitionist propositional calculus. Journal of Symbolic Logic, 27(4), 391-399.

Johansson, I. (1937). Der Minimalkalkül, ein reduzierter intuitionistischer Formalismus. Compositio Mathematica, 4, 119-136.

Kanger, S. (1955). A note on partial postulate sets for propositional logic. Theoria, 21, 99-104.

Kripke, S. A. (1965). Semantical analysis of intuitionist logic I. In Crossley , J. N. and Dummett, M. A. E., editors. Formal Systems and Recursive Functions. Amsterdam, The Netherlands: North Holland, pp. 92-129.

Leblanc, H. (1982). Existence, Truth, and Provability, Albany, NY: State University of New York Press.

Leblanc, H., \& Thomason, R. H. (1966). The demarcation line between intuitionist logic and classical logic. Zeitschrift für Mathematische Logik und Grundlagen der Mathematik, 12, 257-262. Reprinted in Existence, Truth, and Provability. Albany, NY: State University of New York Press, pp. 397-403.

Łukasiewicz, J., \& Tarski, A. (1930). Untersuchungen über den Ausagenkalkül. Comptes rendues des séances de la Société des Sciences et des Lettres de Varsovie, 23, cl. iii, 30-50. Translated as Investigations into the sentential calculus in Tarski (1983). Logic, Semantics and Metamathematics (second edition), Corcoran, J., editor. Indianapolis, IN: Hackett, pp. 38-59.

Parigot, M. (1993). Church-Rosser property in classical free deduction. In Huet, G. and Plotkin, G., editors. Logical Environments. Cambridge, UK: Cambridge University Press, pp. 273-296.

Parsons, C. (1966). A propositional calculus intermediate between the minimal calculus and the classical. Notre Dame Journal of Formal Logic, 7, 353-358.

Prawitz, D. (1965). Natural Deduction: A Proof-Theoretical Study. Stockholm, Sweden: Almqvist \& Wiksell. Reprinted with new preface, Mineola, NY: Dover Publications, 2006.

Prawitz, D. (1979). Proofs and the meaning and completeness of the logical constants. In Hintikka, J., Niiniluoto, I., and Saarinen, E., editors. Essays on Mathematical and Philosophical Logic. Dordrecht, The Netherlands : Reidel.

Prior, A. N. (1962). Formal Logic (second edition). Oxford, UK: Clarendon Press.

Segerberg, K. (1968). Propositional logics related to Heyting's and Johansson's. Theoria, 34, 26-61.

Seldin, J. (1986). On the proof theory of the intermediate logic MH. Journal of Symbolic Logic, 51(3), 626-647.

Stålmarck, G. (1991). Normalization theorems for full first order classical natural deduction. Journal of Symbolic Logic, 56(1), 129-149. 
Tennant, N. (1978). Natural Logic. Edinburgh, Scotland: Edinburgh University Press. Reprinted with corrections, 1990.

Tennant, N. (1997). The Taming of the True. Oxford, UK: Clarendon Press.

Thomas, I. (1960). Independence of Tarski's Law in Henkin's propositional fragments. Notre Dame Journal of Formal Logic, 1(1-2), 74-78.

Thomason, R. H. (1970). On the strong semantical completeness of the intuitionist predicate calculus. Journal of Symbolic Logic, 33(1), 1-7.

Troelstra, A. S., \& Schwichtenberg, H. (1996). Basic Proof Theory. Cambridge, UK: Cambridge University Press.

Zimmermann, E. (2002). Peirce's Rule in natural deduction. Theoretical Computer Science, 275(1-2), 561-574.

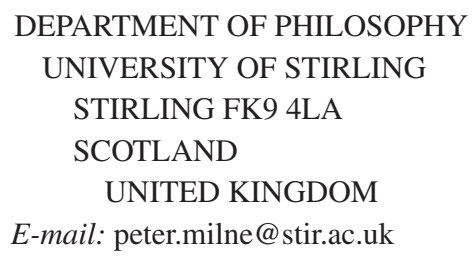

E-mail: peter.milne@stir.ac.uk 